International Journal of Modern Physics B (C) World Scientific Publishing Company

\title{
CONTROL OF THE CASIMIR FORCE USING SEMICONDUCTOR TEST BODIES
}

\author{
G. L. Klimchitskaya \\ North-West Technical University, Millionnaya Street 5, St.Petersburg, 191065, Russia \\ U. Mohideen \\ Department of Physics and Astronomy, University of California, Riverside, California 92521, \\ $U S A$ \\ V. M. Mostepanenko \\ Noncommercial Partnership "Scientific Instruments", Tverskaya Street 11, Moscow, 103905, \\ Russia \\ Received Day Month Year
}

\begin{abstract}
We describe experimental and related theoretical work on the measurement of the Casimir force using semiconductor test bodies. This field of research started in 2005 and several important and interesting results have already been obtained. Specifically, the Casimir force or its gradient were measured in the configuration of an Au-coated sphere and different semiconductor surfaces. It was found that the force magnitude depends significantly on the replacement of the metal with a semiconductor and on the concentration of charge carriers in the semiconductor material. Special attention is paid to the experiment on the optical modulation of the Casimir force. In this experiment the difference Casimir force between an Au-coated sphere and Si plate in the presence and in the absence of laser light was measured. Possible applications of this experiment are discussed, specifically, for the realization of the pulsating Casimir force in three-layer systems. Theoretical problems arising from the comparison of the experimental data for the difference Casimir force with the Lifshitz theory are analyzed. We consider the possibility to control the magnitude of the Casimir force in phase transitions of semiconductor materials. Experiments on measuring the Casimir force gradient between an Au-coated sphere and $\mathrm{Si}$ plate covered with rectangular corrugations of different character are also described. Here, we discuss the interplay between the material properties and nontrivial geometry and the applicability of the proximity force approximation. The review contains comparison between different experiments and analysis of their advantages and disadvantages.
\end{abstract}

Keywords: Casimir effect; semiconductor; charge carrier density.

\section{Introduction}

Historically the Casimir force was discovered 1 as a universal attraction between two parallel electrically neutral ideal metal plates in vacuum separated with a gap of 
width $a$. This force per unit area of the plates (i.e., the pressure) is given by

$$
P(a)=-\frac{\pi^{2} \hbar c}{240 a^{4}}
$$

i.e., it depends only on the Planck constant $\hbar$, velocity of light $c$ and does not depend on the electric charge or any other interaction constants. According to quantum field theory the energy of the electromagnetic field in the vacuum state in free space is infinitely large and all physical energies are measured from the energy of vacuum. If two ideal metal planes are placed in free space, the tangential component of the electric field and the normal component of the magnetic induction must vanish on the planes. As a result, not all zero-point oscillations, whose energies taken together create the infinite vacuum energy, are allowed. In spite of the fact that the energy of allowed oscillations is still equal to infinity, after subtraction of the vacuum energy in free space, one arrives at the finite energy per unit area

$$
E(a)=-\frac{\pi^{2} \hbar c}{720 a^{3}}
$$

It is obvious that the Casimir pressure (1) is obtainable as the negative derivative of Eq. (2) with respect to separation.

The expressions (11) and (2) are of somewhat academic character because they are derived using the idealization of ideal metal planes. Nevertheless, in a few decades after Casimir's discovery a lot of work has been done on the generalization of these results on, for instance, ideal metal rectangular boxes, spheres, cylinder and more complicated configurations, including fields of different spin. $2-5]$ In so doing, it was found that the Casimir force can be both attractive and repulsive (as, for instance, for spheres and rectangular boxes with some specific ratio of sides). A breakthrough was achieved by Lifshitz $\frac{6 \mid 7}{6}$ who generalized the Casimir force for the case of two thick plates made of real materials decribed by the frequency-dependent dielectric permittivities $\varepsilon^{(1,2)}(\omega)$ and developed the unified theory of the van der Waals and Casimir force. According to the Lifshitz theory, the free energy (per unit area) of the dispersion interaction of two material semispaces separated with a gap of width $a$ at temperature $T$ in thermal equilibrium is given by

$$
\mathcal{F}(a, T)=\frac{k_{B} T}{2 \pi} \sum_{l=0}^{\infty} \int_{0}^{\infty} k_{\perp} d k_{\perp} \sum_{\alpha} \ln \left[1-r_{\alpha}^{(1)} r_{\alpha}^{(2)} e^{-2 a q_{l}}\right] .
$$

Here, $k_{B}$ is the Boltzmann constant, $k_{\perp}$ is the magnitude of the projection of the wave vector on the boundary plane $(x, y), q_{l}^{2}=k_{\perp}^{2}+\xi_{l}^{2} / c^{2}, \xi_{l}=2 \pi k_{B} T l / \hbar$ with $l=0,1,2, \ldots$ are the Matsubara frequencies, and the primed summation sign means that the term with $l=0$ is multiplied by $1 / 2$. The reflection coefficients on the first and second plates $(n=1,2)$ for the electromagnetic waves with transverse magnetic $(\alpha=\mathrm{TM})$ and transverse electric $(\alpha=\mathrm{TE})$ polarizations calculated at 
the imaginary Matsubara frequencies are

$$
\begin{aligned}
r_{\mathrm{TM}}^{(n)} & \equiv r_{\mathrm{TM}}^{(n)}\left(i \xi_{l}, k_{\perp}\right)=\frac{\varepsilon^{(n)}\left(i \xi_{l}\right) q_{l}-k_{l}^{(n)}}{\varepsilon^{(n)}\left(i \xi_{l}\right) q_{l}+k_{l}^{(n)}}, \\
r_{\mathrm{TE}}^{(n)} & \equiv r_{\mathrm{TE}}^{(n)}\left(i \xi_{l}, k_{\perp}\right)=\frac{q_{l}-k_{l}^{(n)}}{q_{l}+k_{l}^{(n)}},
\end{aligned}
$$

where

$$
k_{l}^{(n)} \equiv k_{l}^{(n)}\left(i \xi_{l}, k_{\perp}\right)=\left[k_{\perp}^{2}+\varepsilon^{(n)}\left(i \xi_{l}\right) \frac{\xi_{l}^{2}}{c^{2}}\right]^{1 / 2} .
$$

The pressure of the dispersion interaction is expressed as

$$
\begin{aligned}
P(a, T) & =-\frac{\partial \mathcal{F}(a, T)}{\partial a} \\
& =-\frac{k_{B} T}{\pi} \sum_{l=0}^{\infty} \int_{0}^{\infty} q_{l} k_{\perp} d k_{\perp} \sum_{\alpha}\left[\frac{e^{2 a q_{l}}}{r_{\alpha}^{(1)} r_{\alpha}^{(2)}}-1\right]^{-1} .
\end{aligned}
$$

The Lifshitz formulas (3) and (4) were originally derived using an assumption that dielectric materials are characterized by randomly fluctuating sources of electromagnetic fields. The pressure between semispaces was found as the $z z$ component of the Maxwell energy-momentum tensor statistically averaged using the fluctuation-dissipation theorem. Later Eq. (3) was rederived $\sqrt{5[8,10}$ in Casimir's spirit as the finite difference of two infinite free energies of the fluctuating electromagnetic field in the presence of two semispaces with the standard continuity boundary conditions on their surfaces and in the free space. It was shown $\frac{517}{\text { that }}$ in the limiting case of short separation distances between the semispaces (well below the characteristic absorption wavelength of the semispace materials) the free energy and the force of dispersion interaction coincide with the commonly known nonrelativistic van der Waals energy and force.

When materials of semispaces are characterized by the zero temperature, i.e., $T=0 \mathrm{~K}$, the Matsubara frequencies $\xi_{l}$ are replaced with a continuous frequency $\xi$ and the sum over the Matsubara frequencies is replaced with an integral:

$$
k_{B} T \sum_{l=0}^{\infty} \rightarrow \frac{\hbar}{2 \pi} \int_{0}^{\infty} d \xi
$$

In so doing the quantities $q_{l}$ and $k_{l}^{(n)}$ are replaced for continuous quantities $q$ and $k^{(n)}$, and the Lifshitz formula for the free energy (3) turns into the energy at zero temperature

$$
E(a)=\frac{\hbar}{4 \pi^{2}} \int_{0}^{\infty} d \xi \int_{0}^{\infty} k_{\perp} d k_{\perp} \sum_{\alpha} \ln \left[1-r_{\alpha}^{(1)} r_{\alpha}^{(2)} e^{-2 a q}\right] .
$$

In a similar way, Eq. (6) for the Casimir pressure turns into

$$
P(a)=-\frac{\hbar}{2 \pi^{2}} \int_{0}^{\infty} d \xi \int_{0}^{\infty} q k_{\perp} d k_{\perp} \sum_{\alpha}\left[\frac{e^{2 a q}}{r_{\alpha}^{(1)} r_{\alpha}^{(2)}}-1\right]^{-1} .
$$


Here, the reflection coefficients preserve their form (44) with the replacements indicated above. For metallic semispaces at large separations, where relativistic retardation effects are dominant, the force per unit area (9) and energy (8) of dispersion interaction coincide ${ }^{4|5| 12}$ with the Casimir results (1) and (2), respectively. Note that Eqs. (8) and (9) are sometimes used for the interpretation of experiments performed at room temperature $T=300 \mathrm{~K}$. This approach is not self-consistent because the dielectric permittivities at room temperature are substituted into the Lifshitz formulas at zero temperature. In addition it was shown 11 that the quantity obtained from (8) in this way does not coincide with the energy, as it is defined in thermodynamics.

Most of theoretical and experimental work in the Casimir effect was done for metallic test bodies $-3-5]$ It was experimentally demonstrated $13-16$ that at separations below a micrometer the Casimir force is strongly influenced by conduction electrons (the effect of skin depth) and by the surface roughness. By and large metallic bodies have major advantages in comparison with dielectric ones as they ensure low electrostatic charges and low residual potential difference between the surfaces. Actually, a test body coated with a metal film of a few tens of nanometers thickness acts like it is made of a thick metal. Experiments on measuring the gradient of the Casimir force between an Au coated sphere and an Au coated plate 5|16-20 (using the proximity force approximation, this quantity can be recalculated as the Casimir pressure between two parallel plates) led to important conclusions on the nature of interaction between thermal electromagnetic fluctuations and metals. Specifically, it was demonstrated that the experimental data exclude theoretical predictions of the Lifshitz theory at a $99.9 \%$ confidence level if relaxation properties of conduction electrons are taken into account. On the theoretical side, it was shown 1121 that inclusion of relaxation properties of conduction electrons into the Lifshitz theory results in a violation of the Nernst heat theorem. From this it was concluded 22 that there may be some deep differences in the response of a physical system to real and fluctuating electromagnetic fields.

Although metals are most convenient for high precision experiments on the Casimir force, measurements of dispersion forces using semiconductor test bodies open new opportunities for both fundamental physics and numerous applications. It is common knowledge that semiconductors are the most important materials used in nanotechnology, and their conductivity properties range from metallic to dielectric. An aim of considerable scientific and technological promise is to control the magnitude of the Casimir force varying it from large to small, and, if possible, even changing attraction for repulsion and vice versa. The use of semiconductor test bodies suggests several ways on how to achieve this aim. The reflectivity of a semiconductor surface can be changed over a wide frequency range by changing the density of charge carriers. The latter can be done in a number of ways, for instance, through variation of the temperature, using different kinds of doping, or via illumination of the semiconductor surface with laser light. This makes it possible 
to examine the influence of semiconductor material properties on the Casimir force and use the obtained results to modulate the magnitude and separation dependence of the force. We note that in comparison to dielectrics, semiconductors with a reasonably high density of charge carriers have the same advantage as metals, i.e., they do not have problems such as accumulation of charges and screening effects.

An attempt to measure dispersion forces on a semiconductor surface and to modify them by light was undertaken long agd 23 in the configuration of a glass lens and a Si plate and between a glass lens coated with amorphous Si and a Si plate. However, no force change was found on illumination at separations below $350 \mathrm{~nm}$, where it should be most pronounced. This might be explained by the fact that the use of glass (and also high resistivity $\mathrm{Si}$ ) leads to uncontrolled electric forces. The influence of laser light on the Casimir force between two Si plates and between an Au sphere and a Si plate was investigated theoretically!24|25

The modern stage in the investigation of dispersion forces using semiconductor surfaces started in 2005 when the Casimir attraction between a B-doped Si plate and a metal coated sphere was measured 26 using an atomic force microscope (AFM). At this stage, most of experiments were performed in the configuration of an Au-coated sphere (or a spherical lens) above a semiconductor plate drawing on experience accumulated in previous measurements of the Casimir force between metallic test bodies. The present review collects and discusses all main results on the Casimir force obtained using semiconductor surfaces. Section 2 is devoted to the Casimir force between a spherical surface and a plate made of different semiconductors. We

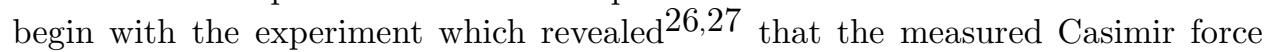
for a plate made of $p$-type $\mathrm{Si}$ is markedly different from the calculation results for high-resistivity dielectric Si. In this experiment it was demonstrated that the density of charge carriers in a semiconductor material influences the Casimir force between metallic and semiconductor test bodies. Then we discuss the experiment where the Casimir force between an Au-coated sphere and two $n$-type Si plates with different densities of charge carriers was successively measured.28 Through this, the difference in the Casimir forces for different Si plates was calculated, and, thus, more clear evidence for the effect of the charge carrier density on the Casimir force was provided. We next consider the experiment on measuring the Casimir force between a metal coated sphere and an indium tin oxide (ITO) plate ${ }^{29130}$ In this experiment, the reduction of the Casimir force by up to a factor of two, when compared with the case of two test bodies made of good metal, was reported. In the end of the section we describe the experiment 31 on measuring the Casimir force between a Ge spherical lens with the curvature radius $15.1 \mathrm{~cm}$ and Ge plate. The measurement data of this experiment were found consistent 31 with five different theoretical approaches to the thermal Casimir force. In this connection we indicate a possible source of noncontrolled systematic errors inherent to all measurements of the Casimir force using lenses of centimeter-size curvature radii.

In Section 3 the experiment on the optical modulation $\underline{32 \mid 33}$ of the Casimir force is considered. Here, the difference in the Casimir forces between an Au-coated sphere 
and a Si plate in the presence and in the absence of laser light on the plate is measured. The frequency and power of the laser pulses are selected in such a way that the charge carrier density in Si during the bright phase is almost 5 orders of magnitude higher than in the dark phase. In so doing, $\mathrm{Si}$ in the bright phase was a metal-type semiconductor (conductivity of such a material goes to a nonzero limit with vanishing temperature). In the dark phase $\mathrm{Si}$ was a semiconductor of dielectric-type with vanishing conductivity in the limit of zero temperature. The comparison between the measured difference Casimir force and the Lifshitz theory has led to an unexpected conclusion. It was demonstrated that the Lifshitz theory with inclusion of dc conductivity of $\mathrm{Si}$ in the dark phase is excluded by the data at a 95\% confidence level. At the same time the measurement data were found consistent with the Lifshitz theory if the dc conductivity of Si in the dark phase is omitted. Keeping in mind that dc conductivity is connected with the relaxation properties of free charge carriers, one can conclude that for dielectric-type semiconductors the optical modulation experiment plays the same role as the experiments $17-20$ mentioned above for metals. In both cases the experimental data are inconsistent with the Lifshitz theory taking into account relaxation properties of free charge carriers. From the theoretical side it was proved $34-36$ that the inclusion of the dc conductivity in the model of the dielectric response for dielectrics and dielectric-type semiconductors results in contradiction between the Lifshitz theory and the Nernst heat theorem. This is also somewhat analogous with the respective situation for metals. In Sec. 3 we also discuss recent attempts $37-39$ to modify the Lifshitz theory and show that they are excluded $40-43$ by the results of the optical modulation experiment at a $70 \%$ confidence level. Thus, the optical modulation experiment, which demonstrated the possibility to periodically change the Casimir force with light, is important not only for applications, but for fundamental physics as well.

Section 4 is devoted to the investigation of the changes of the Casimir force in phase transitions. Experiments on this subject involve an $\mathrm{Au}$-coated sphere and a plate made of a phase change material. In the previous experiment $\frac{44}{4}$ an amorphous sample of AgInSbTe was used as the plate. Under annealing the plate material was switched from the amorphous to crystalline phase. In the proposed experiment $\frac{45}{45}$ the $\mathrm{VO}_{2}$ film deposited on a sapphire plate undergoes dielectric-to-metal phase transition with an increase in temperature.

In Sec. 5 we discuss measurements of the gradient of the Casimir force between an Au-coated sphere and a Si surface covered with rectangular corrugations. $\frac{46}{47}$ These measurements allow to observe effects that include the role of geometry and the optical properties of a semiconductor material.

The possibility to obtain a pulsating Casimir force in a three layer system, where at least one layer is made of a semiconductor, is considered in Sec. 6. A pulsation, i.e., periodic change of attraction with repulsion and vice versa, can be achieved $\frac{48}{4}$ through the illumination of a semiconductor layer with laser pulses.

In Sec. 7 the reader will find our conclusions and discussion.

The presentation below is based on original publications. In several cases, how- 
ever, we make a more exact analysis of the experimental data and their comparison with theory. These cases are indicated in the text. Many figures illustrating the comparison between experiment and theory are made specially for this review. We write equations in the Gaussian system of units. Some values of the experimentally measured quantities are given, however, in the International System of units.

\section{Casimir force between a spherical surface and a plate made of different semiconductor materials}

Here, we discuss the results of four experiments, where a semiconductor plate interacts via the Casimir force with an Au-coated sphere or a $\mathrm{Ge}$ spherical lens. In these experiments, different semiconductors are used. In all cases it was confirmed that the replacement of the metallic plate with a semiconductor one markedly diminishes the magnitude of the Casimir force. However, as is shown below, different experiments, depending on their precision, contain different information with respect to theoretical models consistent with the experimental data.

\subsection{B-doped Si plate}

In this experimen, 2627 the Casimir force was measured between an Au-coated sphere of diameter $2 R=202.6 \pm 0.3 \mu \mathrm{m}$ and a single-crystal Si plate doped with B. The $p$-type Si plate had an area $5 \times 10 \mathrm{~mm}^{2}$ and a thickness of $350 \mu \mathrm{m}$. The resistivity of the plate at room temperature $T=300 \mathrm{~K}$ was $\rho=0.0035 \Omega \mathrm{cm}$ which corresponds to a charge carrier density $n \approx(2.9-3.2) \times 10^{19} \mathrm{~cm}^{-3}$. This is a metaltype semiconductor whose conductivity does not go to zero when the temperature vanishes. For typical metals, however, resistivities are two or three orders of magnitude lower. The same experimental setup $\frac{13-16}{-16}$ as for two metallic test bodies (with much higher vacuum of $2 \times 10^{-7}$ Torr) was used to measure the Casimir force. The schematic of the setup is shown in Fig. 1, where force between the sphere and the plate causes the cantilever of an AFM to flex. This flexing is detected by the deflection of the laser beam leading to a difference signal between the photodiodes $\mathrm{A}$ and $\mathrm{B}$. The difference signal is calibrated by means of an electrostatic force. Here we only discuss the obtained experimental results and their comparison with theory and do not consider details of the setup and calibration procedures which are fully described in the original publications $26 \mid 27$ and in Refs. [5, 16.

The Casimir force between the sphere and the plate was measured as a function of separation. In so doing, the sphere was kept grounded while a compensating voltage was applied to the plate to cancel the residual potential difference $V_{0}=-0.114 \pm 0.002 \mathrm{~V}$ (the latter was found to be independent of separation). The separation was varied continuously from about $6 \mu \mathrm{m}$ to small distances by applying continuous triangular voltages at $0.02 \mathrm{~Hz}$ to the piezoelectric actuator. However, the measurement data becomes meaningful only below $350 \mathrm{~nm}$ (at $a=350 \mathrm{~nm}$ the total experimental error is approximately equal to the force magnitude). The force data were collected at equidistant points $a \geq 62.33 \mathrm{~nm}$ separated by $0.17 \mathrm{~nm}$. The 


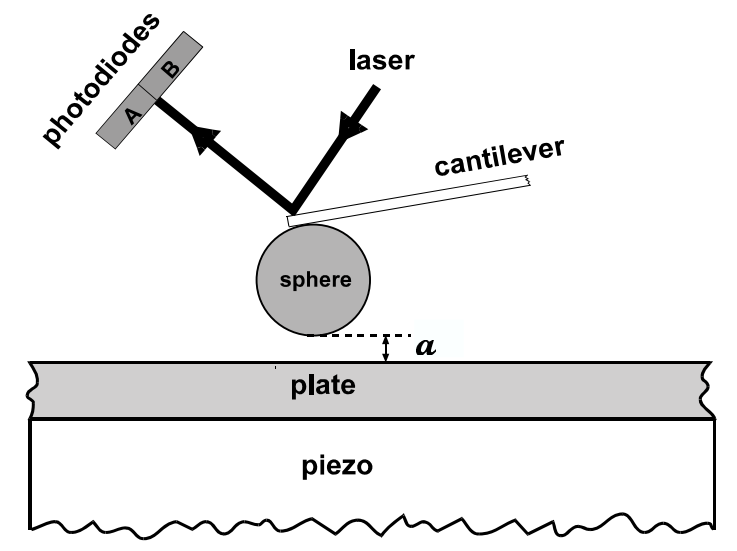

Fig. 1. Schematic diagram of the experimental setup using an atomic force microscope.

mesurement was repeated 65 times. The mean force $\bar{F}^{\text {expt }}$ is shown in Fig. 2 as a function of separation. The total experimental error of the force measurements, $\Delta^{\text {tot }} F^{\text {expt }}$, determined at a $95 \%$ confidence level, was equal to $3.33 \mathrm{pN}$. It is mostly determined by the random error. The error in the measurement of absolute separation was $\Delta a=0.8 \mathrm{~nm}$.

Now we compare the experimental results with theory. In the original publications,, 26127 as well as in Refs. 15, 16, the theoretical Casimir forces for the needs of this experiment were computed at $T=0$, whereas the measurements were performed at $T=300 \mathrm{~K}$. This was justified by the smallness of thermal effects at short separations. Keeping in mind increased attention to the problem of thermal Casimir force during the last few years, here we recalculate theoretical results using

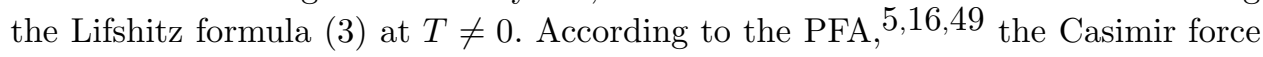
between a sphere and a plate is given by

$$
F(a, T)=2 \pi R \mathcal{F}(a, T),
$$

where the free energy $\mathcal{F}(a, T)$ is given in Eq. (3). Errors arising from the use of an approximate Eq. (10) are less $\frac{50}{1}$ than $a / R \approx 0.1 \%$ at separations of about $100 \mathrm{~nm}$.

For the dielectric permittivity of $\mathrm{Au}, \varepsilon^{(1)}\left(i \xi_{l}\right)$ in Eq. (3), we use the generalized plasma-like model

$$
\varepsilon^{(1)}\left(i \xi_{l}\right)=\frac{\omega_{p}^{(1)^{2}}}{\xi_{l}^{2}}+\varepsilon_{\mathrm{ce}}^{(1)}\left(i \xi_{l}\right)
$$

where $\varepsilon_{\mathrm{ce}}^{(1)}\left(i \xi_{l}\right)$ is the dielectric permittivity determined by the core electrons, and $\omega_{p}^{(1)}=9.0 \mathrm{eV}$ is the plasma frequency. At frequencies below $\xi=15 \mathrm{eV}$ the analytical expression for $\varepsilon_{\mathrm{ce}}^{(1)}\left(i \xi_{l}\right)$ in terms of the six-oscillator model is available. $5[16 \mid 20$ This allows precise computations of the Casimir force (10) at $a \geq 100 \mathrm{~nm}$. At shorter separations, a more precise representation for $\varepsilon_{\mathrm{ce}}^{(1)}\left(i \xi_{l}\right)$ is required. To obtain this, one 


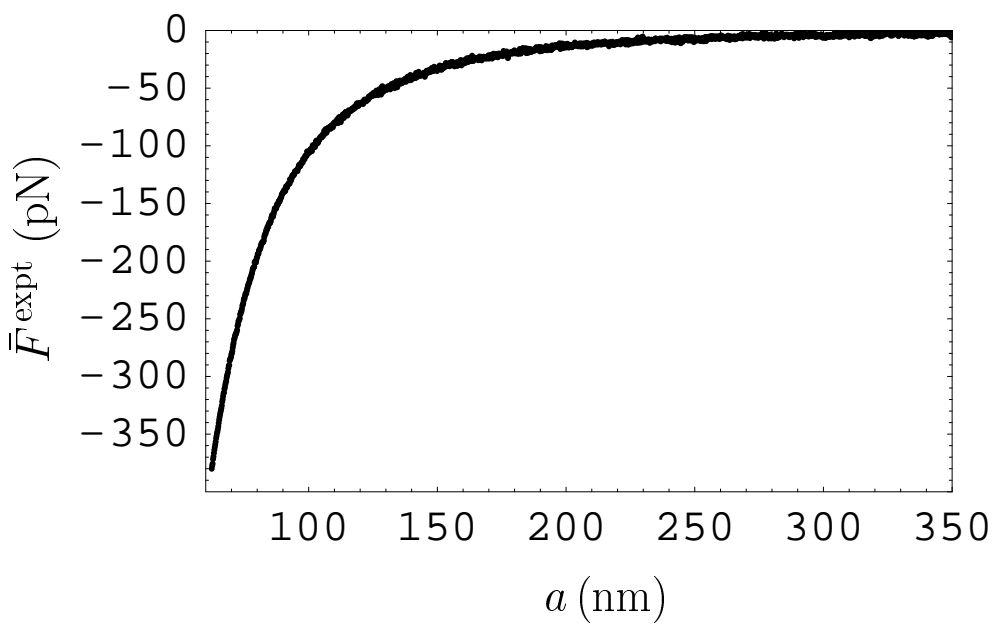

Fig. 2. The mean measured Casimir force between an Au-coated sphere of $101.3 \mu \mathrm{m}$ radius and Si plate of $0.0035 \Omega \mathrm{cm}$ resistivity is shown as the function of separation.

should first consider $\operatorname{Im} \varepsilon_{\text {ce }}^{(1)}(\omega)$ which is the difference between $2 n_{1}(\omega) n_{2}(\omega)$ [where $n_{1}(\omega)$ and $n_{2}(\omega)$ are the tabulated data for real and imaginary parts of the complex index of refraction of $\mathrm{Au}$ at different frequencies 51 and the respective contribution from conduction electrons. Note that $\operatorname{Im} \varepsilon_{\mathrm{ce}}^{(1)}(\omega)$ vanishes at low frequencies. Then $\varepsilon_{\mathrm{ce}}^{(1)}\left(i \xi_{l}\right)$ is found from the Kramers-Kronig relation

$$
\varepsilon_{\mathrm{ce}}^{(1)}\left(i \xi_{l}\right)=1+\frac{2}{\pi} \int_{0}^{\infty} \frac{\omega \operatorname{Im} \varepsilon_{\mathrm{ce}}^{(1)}(\omega)}{\omega^{2}+\xi_{l}^{2}} d \omega
$$

A frequently used alternative method to find the dielectric permittivity of $\mathrm{Au}$ along the imaginary frequency axis is based on the extrapolation of the optical data by means of the Drude dielectric function

$$
\varepsilon_{D}^{(1)}(\omega)=1-\frac{\omega_{p}^{(1)^{2}}}{\omega\left(\omega+i \gamma^{(1)}\right)},
$$

where $\gamma^{(1)}=0.035 \mathrm{eV}$ is the relaxation parameter. In the framework of this method 52 the tabulated data for $2 n_{1}(\omega) n_{2}(\omega)$ are extrapolated for low frequencies by using the imaginary part of $\varepsilon_{D}^{(1)}(\omega)$ defined in Eq. (13). Then the dielectric permittivity of $\mathrm{Au}$ at the imaginary Matsubara frequencies, $\tilde{\varepsilon}^{(1)}\left(i \xi_{l}\right)$, is obtained by means of the standard Kramers-Kronig relation.

The dielectric permittivities $\varepsilon^{(1)}\left(i \xi_{l}\right)$ and $\tilde{\varepsilon}^{(1)}\left(i \xi_{l}\right)$ for Au are shown in Fig. 3 by the solid and dashed black lines, respectively. The dashed line takes into account the relaxation properties of conduction electrons, whereas the solid line disregards relaxation of free charge carriers. As was noted in Sec. 1, experiments with metallic test bodies $5\left[16-20\right.$ exclude the permittivity $\tilde{\varepsilon}^{(1)}\left(i \xi_{l}\right)$. Keeping in mind that the latter describes correctly the dielectric response of $\mathrm{Au}$ for a real electromagnetic field, this 


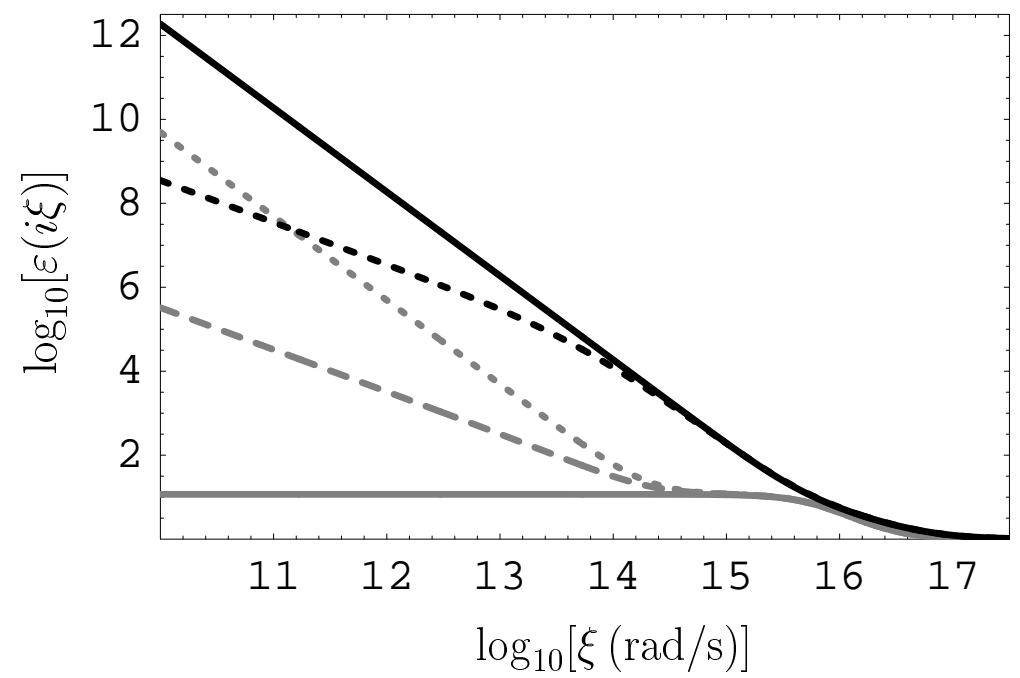

Fig. 3. The logarithms of the dielectric permittivity as a function of the imaginary frequency for $\mathrm{Au}$ described by the generalized plasma-like model (the solid black line), for Au described by the tabulated optical data extrapolated by the Drude model (the dashed black line), for high-resistivity $\mathrm{Si}$ (the solid grey line), for Si described by the generalized plasma-like model (14) (the dotted grey line), and for Si described by the Drude-like model (15) (the dashed grey line).

might be connected with some deep unexplored differences between real and fluctuating fields. In the experiment under consideration in this section the separation region of main interest is from 62 to $100 \mathrm{~nm}$ (using the force magnitudes in Fig. 2 , one can conclude that the relative total experimental error varies from only $0.87 \%$ at the shortest separation to $5.3 \%$ at $a=120 \mathrm{~nm}$ and reaches $64 \%$ at $a=300 \mathrm{~nm}$ ). In this separation region the use of the dielectric permittivities $\varepsilon^{(1)}\left(i \xi_{l}\right)$ and $\tilde{\varepsilon}^{(1)}\left(i \xi_{l}\right)$ leads to only negligible differences in the Casimir force specified below.

The dielectric permittivity of dielectric Si with resistivity $\rho_{0}=1000 \Omega \mathrm{cm}$ is obtained using the tabulated optical data ${ }^{51}$ for the complex index of refraction and the Kramers-Kronig relation. It is notated as $\varepsilon_{\mathrm{ce}}^{(2)}\left(i \xi_{l}\right)$ and shown by the solid grey line in Fig. 3. Note that the analytical approximations to $\varepsilon_{\mathrm{ce}}^{(2)}\left(i \xi_{l}\right)$ suggested 25153 lead to errors of less than $1 \%$ in the magnitude of the Casimir force. The resistivity of a B-doped Si plate used in the measurements was much lower, and the doping concentration indicated above corresponds to a plasma frequency $\omega_{p}^{(2)} \approx 7 \times 10^{14} \mathrm{rad} / \mathrm{s}$ and relaxation parameter $\gamma^{(2)} \approx 1.5 \times 10^{14} \mathrm{rad} / \mathrm{s}$. Thus, disregarding the relaxation processes of charge carriers, the dielectric permittivity of the Si plate $\varepsilon^{(2)}(i \xi)$ in Eq. (3) can be represented in the form

$$
\varepsilon^{(2)}(i \xi)=\frac{\omega_{p}^{(2)^{2}}}{\xi^{2}}+\varepsilon_{\mathrm{ce}}^{(2)}(i \xi) .
$$

This is the generalized plasma-like model for metallic-type Si with resistivity $\rho$. Alternatively, preserving the role of relaxation properties of free charge carriers, 
instead of Eq. (14), one obtains

$$
\tilde{\varepsilon}^{(2)}(i \xi)=\frac{\omega_{p}^{(2)^{2}}}{\xi\left(\xi+\gamma^{(2)}\right)}+\varepsilon_{\mathrm{ce}}^{(2)}(i \xi)
$$

The dielectric permittivities of $\mathrm{Si} \varepsilon^{(2)}(i \xi)$ and $\tilde{\varepsilon}^{(2)}(i \xi)$ are shown in Fig. 3 by the dotted and dashed grey lines, respectively.

Finally, for the comparison between experiment and theory one should take the surface roughness into account which is not included in Eqs. (3) and (10). For this purpose the topographies of both Au-coated sphere and Si plate were investigated using an AFM. The fractions of the Au-coating on the sphere with different heights were determined. The same was done for a Si plate. Then the Casimir force was computed using Eqs. (3) and (10) for all fractions of the surfaces and the results were geometrically averaged $[5] 16|26| 27$ to obtain the theoretical Casimir force between rough surfaces $F^{\text {theor }}(a)$. Here, $a$ is the separation distance between the zero levels of the roughness profiles on both surfaces. The contributions to the force from surface roughness were very minor because the rms roughness was only 3.446 and $0.111 \mathrm{~nm}$ on the sphere and on the plate, respectively.

In Fig. 4 we present comparison between the experimental data and different theoretical approaches over the separation regions (a) from 62 to $80 \mathrm{~nm}$ and (b) from 80 to $100 \mathrm{~nm}$. The experimental data for the mean Casimir forces are shown as crosses. The arms of the crosses are equal to twice the respective absolute errors determined at a $95 \%$ confidence level. For both black and grey lines representing the
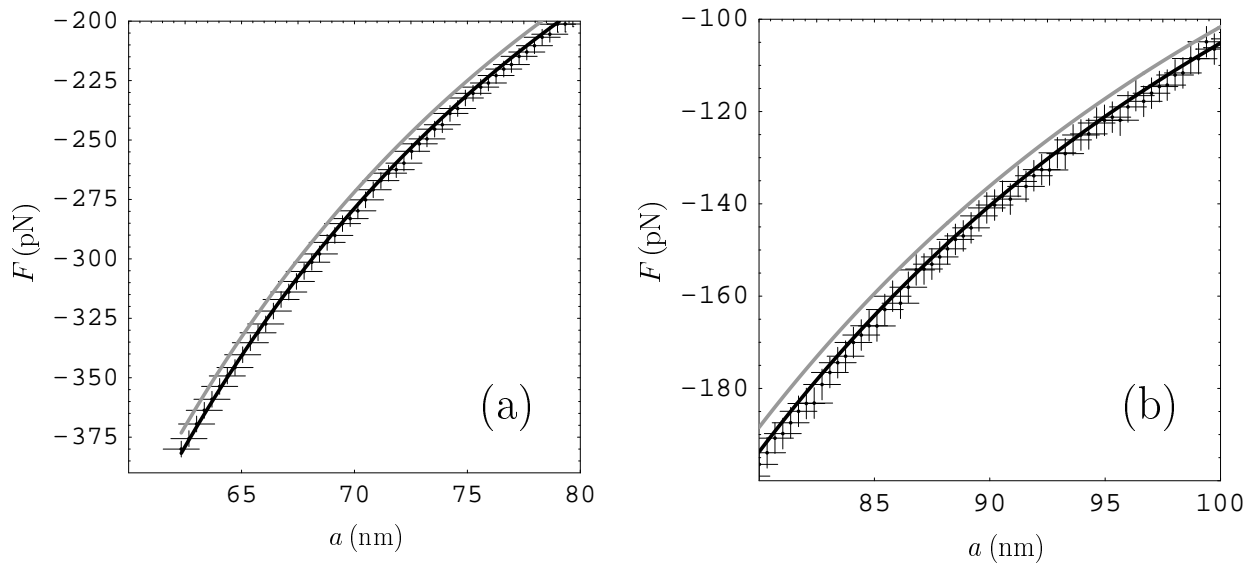

Fig. 4. Comparison between the experimental data for the Casimir force in the configuration of an Au-coated sphere and Si plate shown as crosses and two theoretical approaches over the separation regions (a) from 62 to $80 \mathrm{~nm}$ and (b) from 80 to $100 \mathrm{~nm}$ (only each third cross is shown). The arms of the crosses are determined at a $95 \%$ confidence level. For both the black and grey theoretical lines Au is described by means of the generalized plasma-like model. Si is described either by the generalized plasma-like model (the black line) or as a high-resistivity material (the grey line). 

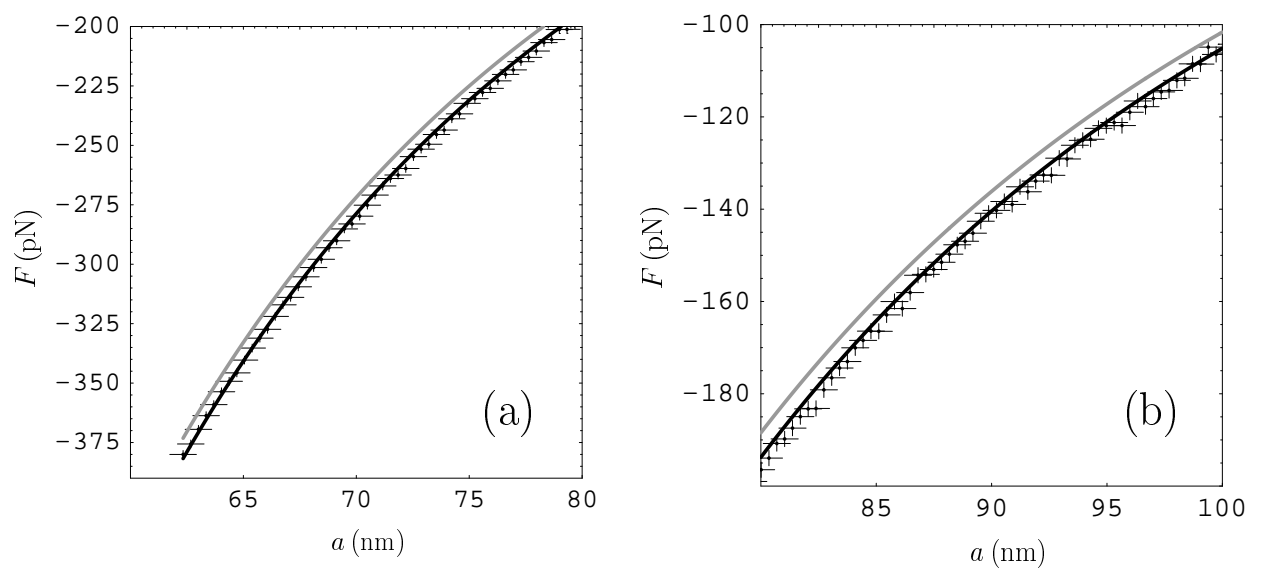

Fig. 5. The same as in Fig. 4 but the arms of the crosses are determined at a $70 \%$ confidence level.

computational results Au sphere is described by the generalized plasma-like model in Eq. (11) (the solid black line in Fig. 3). The Si plate is described either by the generalized plasma-like model of Eq. (14) (the black line) or by the dielectric permittivity $\varepsilon_{\mathrm{ce}}^{(2)}(i \xi)$ of a high resistivity $\mathrm{Si}$ (the grey line). As can be seen in Fig. 4(a), many experimental crosses at least touch the grey line at $a<75 \mathrm{~nm}$. This means that at separations below $75 \mathrm{~nm}$ both theoretical approaches are consistent with the data within a $95 \%$ confidence interval. From Figs. 4(a) and 4(b), however, one can conclude that within the separation interval from 75 to $100 \mathrm{~nm}$ the experimental data are consistent with the description of Si using Eq. (14) (the black line), but

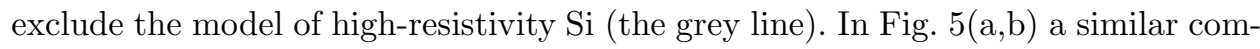
parison between experiment and theory is shown, but the experimental crosses are plotted at a $70 \%$ confidence level. For this purpose the vertical arms of all crosses determined by the random errors were divided by two as follows from the normal or Student distribution. The horizontal arms determined by the systematic errors were divided by 1.357 based on the uniform distribution (see below for a more detailed discussion). From this figure it can be concluded that the model of high-resistivity dielectric $\mathrm{Si}$ is excluded by the data over a wide interval from 70 to $100 \mathrm{~nm}$.

Another method for the comparison between experiment and theory considers differences between the theoretical and mean experimental Casimir forces, $F^{\text {theor }}\left(a_{i}\right)-\bar{F}^{\operatorname{expt}}\left(a_{i}\right)$, and the confidence intervals for these differences taking into account both the experimental and theoretical errors. In Fig. $6(a, b)$ the force differences are indicated as dots and the borders of the confidence intervals determined at a $95 \%$ and $70 \%$ confidence levels are shown as the solid and dashed lines, respectively. Theoretical Casimir forces are computed using the description of $\mathrm{Si}$ (a) by the generalized plasma-like model (14) and (b) the model of high-resistivity Si. Here, the half-widths of the confidence intervals ad a $95 \%$ and $70 \%$ confidence levels are 

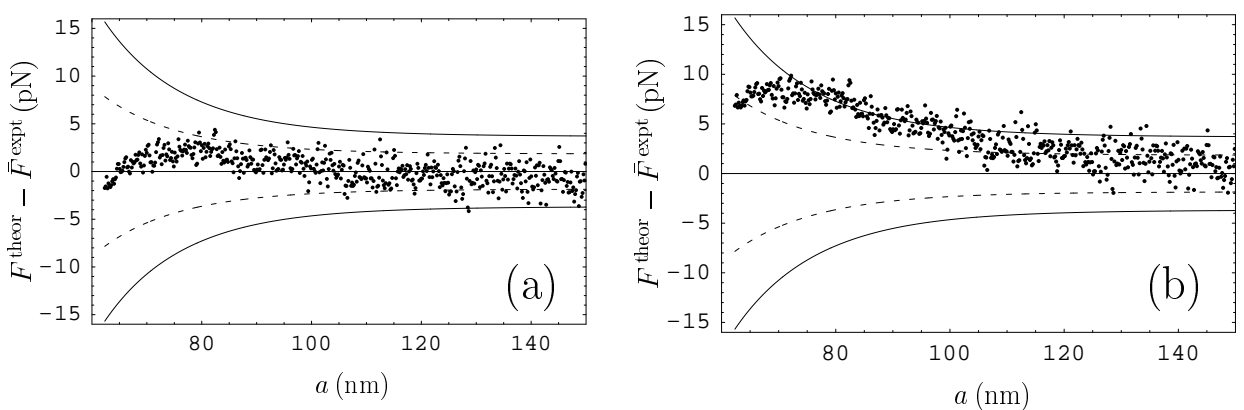

Fig. 6. The differences between theoretical and mean experimental Casimir forces in the configuration of an Au-coated sphere and Si plate are indicated as dots. The borders of the confidence intervals determined at a $95 \%$ and $70 \%$ confidence levels are shown as the solid and dashed lines, respectively. The theoretical Casimir forces are computed using the generalized plasma-like model for $\mathrm{Au}$ and describing $\mathrm{Si}$ by (a) the generalized plasma-like model and (b) the model of highresistivity material.

related as for a normal distribution, i.e., $\Xi_{0.95} / \Xi_{0.70}=2$ because the distribution of the force differences was found to be close to the normal one (this is in fact a conservative assumption as for the Student distribution with different numbers of degrees of freedom $\Xi_{0.95} / \Xi_{0.70}>2$ holds). As can be seen in Fig. 6(a), the most of dots over the entire separation interval from 62 to $150 \mathrm{~nm}$ lie inside both $70 \%$ and 95\% confidence intervals. This means that the description of Si using the generalized plasma-like model (14) is consistent with the data. From Fig. 6(b) it is seen that within the interval from 75 to $115 \mathrm{~nm}$ more than $5 \%$ of dots are outside the $95 \%$ confidence intervals. Thus, within this interval the model of dielectric $\mathrm{Si}$ is excluded by the data at a $95 \%$ confidence level. From Fig. 6(b) one can conclude also that at the $70 \%$ confidence level the model of high-resistivity $\mathrm{Si}$ is excluded by the data within a wider range of separations from 65 to $145 \mathrm{~nm}$ because here more than $30 \%$ of dots are outside the $70 \%$ confidence intervals.

Now we demonstrate that at separations considered the generalized plasma-like model used above for the description of dielectric properties of Au and Si leads to almost the same results as the tabulated optical data extrapolated to low frequencies by means of the Drude model. As an example, at $a=62.33,104.83$, and $147.33 \mathrm{~nm}$ the magnitudes of theoretical Casimir forces between a sphere and a plate, $\left|F^{\text {theor }}\right|$, used in Figs. 4, 5 and 6(a) are the following: 382.760, 92.4342, and $36.0303 \mathrm{pN}$. If, alternatively, the tabulated optical data for both $\mathrm{Au}$ and $\mathrm{Si}$ were extrapolated by the Drude model, we would get at respective separations $\left|\tilde{F}^{\text {theor }}\right|=382.393$, 91.8658 , and $35.5928 \mathrm{pN}$. This leads to only $-0.09 \%,-0.61 \%$ and $-1.21 \%$ relative differences at the same respective separations. These differences are well below the experimental errors in the experiment under consideration.

To conclude this section, the first measurement of the Casimir force with semiconductor plate 2627 convincingly demonstrated that by using semiconductors one can control the force magnitude. If the plate in this experiment were made of 
$\mathrm{Au}$ instead of $\mathrm{Si}$ with all other conditions and parameters preserved, the magnitude of the Casimir force at $a=62.33,104.83$, and $147.33 \mathrm{~nm}$ would be equal to $\left|F_{\mathrm{Au}-\mathrm{Au}}^{\text {theor }}\right|=515.948,134.456$ and $54.8289 \mathrm{pN}$, respectively. Comparing these values with the above magnitudes of the Casimir force between an Au sphere and Si plate, we obtain that through the replacement of Au with Si force is decreased by $25.8 \%$, $31.3 \%$ and $34.3 \%$ at respective separations. It was demonstrated also that charge carrier density in metallic materials is an important characteristic for the determination of the Casimir force. The experiment described in the next section provides further confirmation to these conclusions.

\subsection{Two P-doped Si plates with different dopant concentrations}

In the next experiment 28 on measuring the Casimir force between an Au-coated sphere and a semiconductor plate, two P-doped Si plates which possess radically different charge carrier densities were compared. The scheme of the setup was already described in Sec. 2.1 (see Fig. 11). In this case the diameter of the Au-coated polystyrene sphere was equal to $2 R=201.8 \pm 0.6 \mu \mathrm{m}$. The two Si plates were placed next to each other and had an area $4 \times 7 \mathrm{~mm}^{2}$ and a thickness of $500 \mu \mathrm{m}$. Two identically polished single-crystal Si samples were chosen as plates. They were $n$-type and doped with $\mathrm{P}$. The resistivity of both plates was measured using the four-probe techniques. The plates had a $\rho_{a} \approx 0.43 \Omega \mathrm{cm}$ (i.e., more than two orders of magnitude higher than in the previous experiment with one Si plate). This corresponds to a concentration of charge carriers $n_{a} \approx 1.2 \times 10^{16} \mathrm{~cm}^{-3}$.

One of the plates was used in the experiment as the first Si plate. The other plate was subjected to thermal-diffusion doping in order to decrease the resistivity and to increase the concentration of charge carriers ${ }^{[5] 28}$ As a result, the resistivity and the carrier density were measured to be $\rho_{b} \approx 6.7 \times 10^{-4} \Omega \mathrm{cm}$ and $n_{b} \approx 3.2 \times 10^{20} \mathrm{~cm}^{-3}$. This plate was used as the second Si plate in the experiment. Its resistivity was more than 50 times less than the resistivity of the plate in the experiment described in Sec. 2.1. In fact the first plate was a semiconductor of dielectric-type and the second of metallic-type (in the sense that conductivity of the first goes to zero with vanishing temperature, whereas conductivity of the second has a nonzero limit in the limit of zero temperature).

The calibration procedures using the measurements of electric forces were significantly improved as compared with previous experiments on the Casimir force. Specifically, the parabolic dependence of the signal on the applied voltage was used to obtain the residual potential difference $V_{0}$ between the grounded sphere and

the plate 28154 For the first and second samples the residual potential differences were found to be $V_{0 a}=-0.341 \pm 0.002 \mathrm{~V}$ and $V_{0 b}=-0.337 \pm 0.002 \mathrm{~V}$. Both were demonstrated to be independent on separation within the limits of an experimental error. The separation was varied continuously in the same way as was described in Sec. 2.1 up to some minimum value. The measurement of the Casimir force between the sphere and the first plate was repeated 40 times over the separation region from 

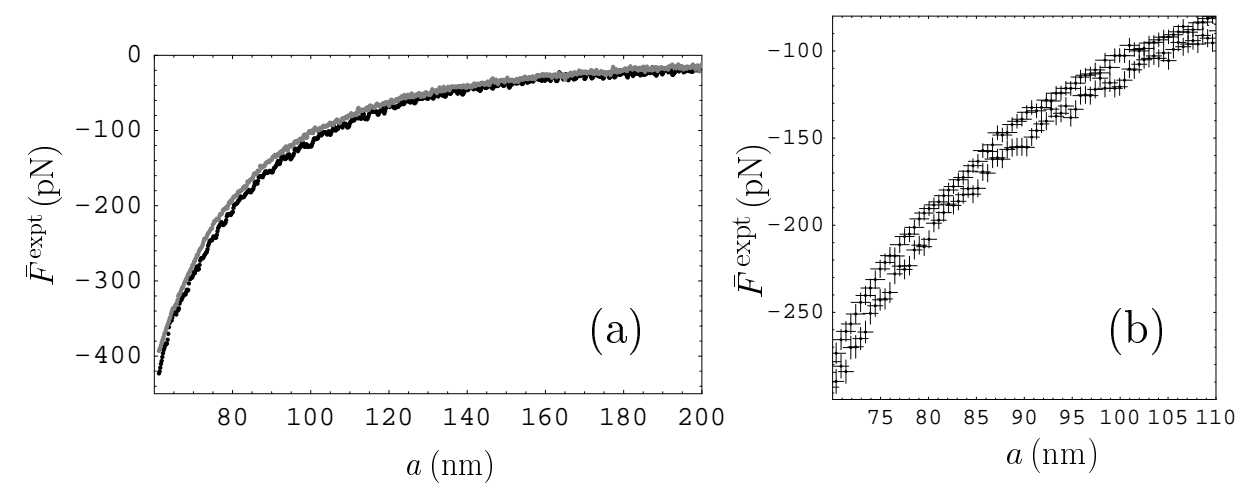

Fig. 7. (a) Mean measured Casimir forces between an Au-coated sphere and Si plates with high (the grey dots) and low (the black dots) resistivity are shown as a function of separation. (b) The same mean forces are shown as crosses with arms determined at a $95 \%$ confidence level over a narrower range of separations (only every third cross is plotted).

$61.19 \mathrm{~nm}$ to $400 \mathrm{~nm}$. The mean values of the obtained force $\bar{F}_{a}^{\text {expt }}$ over the separation region below $200 \mathrm{~nm}$, where the results are most meaningful, are shown in Fig. 17(a) as the grey dots. Similar measurements of the force between the sphere and the second plate was repeated 39 times over the region from $60.51 \mathrm{~nm}$ to $400 \mathrm{~nm}$. The mean values $\bar{F}_{b}^{\text {expt }}$, as a function of separation, are shown in Fig. 7 (a) as the black dots.

The total experimental error in both measurements depends on separation and is determined by the random errors (the systematic error determined at a $95 \%$ confidence level is equal to only $1.2 \mathrm{pN}$ for both measurements and is separationindependent). For example, for a measurement with the first plate (which is of higher resistivity) the total experimental error determined at a $95 \%$ confidence level is equal to 8,6 , and $4 \mathrm{pN}$ at the separations $61.19 \mathrm{~nm}, 70 \mathrm{~nm}$, and $a \geq 80 \mathrm{~nm}$, respectively. For a measurement with the second plate (which is of lower resistivity) the total experimental error determined at the same confidence is equal to 11,7 , and $5 \mathrm{pN}$ at the respective separations $61.51 \mathrm{~nm}, 70 \mathrm{~nm}$, and $a \geq 80 \mathrm{~nm}$. For the first and second plates, separations were measured with the absolute errors equal to 1 and $0.8 \mathrm{~nm}$, respectively.

To make sure that within some separation distance the measurement results for the first and second plates do not overlap, in Fig. 7(b) the data are plotted as crosses. The arms of the crosses are determined by the absolute errors, as explained in Sec. 2.1. From Fig. (7) (b) it can be seen that at most separations within the interval from 70 to $110 \mathrm{~nm}$ the measured Casimir forces between the sphere and the first and second plates belong to two individual lines separated with some gap. Note that only each third experimental point is plotted in Fig. 7 (b) because in the presence of all points the figure becomes unreadable. All crosses are plotted at a $95 \%$ confidence level. 

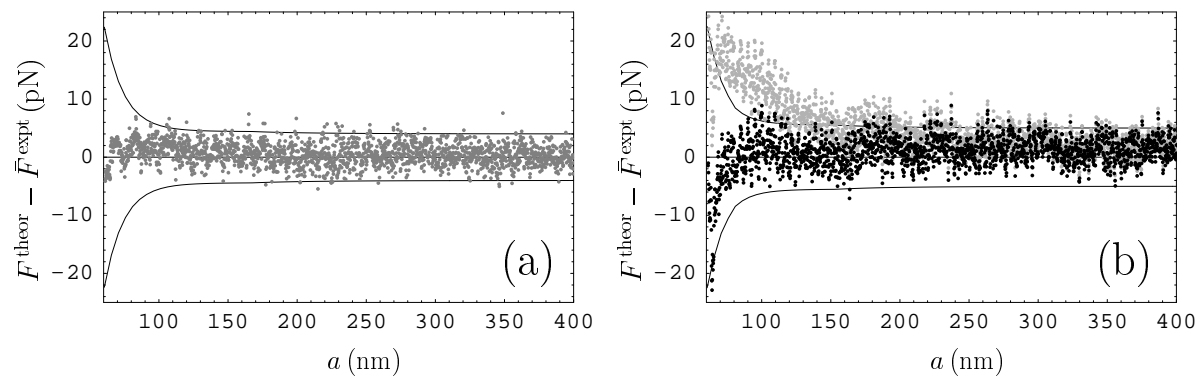

Fig. 8. The differences between theoretical and mean measured Casimir forces in the configuration of an Au-coated sphere and two Si plates are shown as dots for (a) high-resistivity and (b) lowresistivity plate. Au is described by the generalized plasma-like model. Si of the plates is described as a high-resistivity material (the grey dots) or by the generalized plasma-like model (the black dots).

Now we are in a position to compare the experimental data with theory. This can be conveniently done using the second method of comparison considered in Sec. 2.1 which is based on the consideration of differences between theoretical and experimental Casimir forces. The theoretical Casimir forces are computed using Eqs. (3) and (10) and taking surface roughness into account by means of the geometrical averaging, as discussed in Sec. 2.1. In so doing the dielectric permittivity of $\mathrm{Au}, \varepsilon^{(1)}\left(i \xi_{l}\right)$, is presented by Eq. (11). The dielectric permittivity of high-resistivity $\mathrm{Si}$ (the first plate) along the imaginary Matsubara frequencies is given by $\varepsilon_{\mathrm{ce}}^{(2)}(i \xi)$ obtained using the tabulated optical data for the complex index of refraction (see Sec. 2.1). The role of low concentration of charge carriers $n_{a}$ in this experiment is negligibly small. It can be investigated in more precise difference force measurement (see Sec. 3). As to the dielectric permittivity of low-resistivity Si (the second plate), one can describe it either by $\varepsilon_{\mathrm{ce}}^{(2)}(i \xi)$ or by the dielectric permittivity of the generalized plasma-like model $\varepsilon^{(2)}(i \xi)$ defined in Eq. (14) [this experiment, as well as the experiment discussed in Sec. 2.1, is not of sufficient precision to discriminate between theoretical descriptions given by Eqs. (14) and (15)]. The value of the plasma frequency $\omega_{p}^{(2)}=2.0 \times 10^{15} \mathrm{rad} / \mathrm{s}$ should be used in this case in accordance with the concentration of charge carriers. Computations below are done at the laboratory temperature $T=300 \mathrm{~K}$ specially in this review (in the original publication 28 computations were performed at $T=0$ keeping in mind the smallness of thermal effects at short separations).

In Fig. $8(a)$ the differences between computed and mean measured Casimir forces, $F_{a}^{\text {theor }}-\bar{F}_{a}^{\text {expt }}$, for the high-resistivity plate versus separation are shown as grey dots. The pairs of solid lines in Fig. 8(a) indicate the borders of the confidence intervals, to which the differences between theoretical and mean experimental Casimir forces should belong with a $95 \%$ probability. These confidence intervals take into account both the experimental and theoretical errors combined using the con-

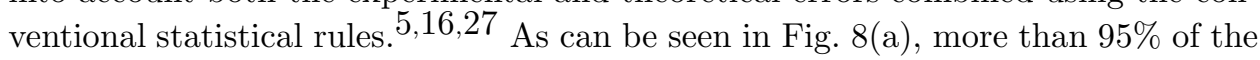


grey dots belong to the confidence intervals. This means that theoretical description of high-resistivity Si by the dielectric permittivity $\varepsilon_{\mathrm{ce}}^{(2)}(i \xi)$ with charge carriers disregarded is consistent with the experimental data within a $95 \%$ confidence interval.

Quite a different situation holds for a low-resistivity sample. In Fig. 8(b) we show as grey dots the theoretical and mean measured force differences, $F_{b}^{\text {theor }}-\bar{F}_{b}^{\text {expt }}$, when the theoretical Casimir forces are computed using the dielectric permittivity $\varepsilon_{\mathrm{ce}}^{(2)}(i \xi)$. In the same figure the black dots represent the force differences, $F_{b}^{\text {theor }}-\bar{F}_{b}^{\text {extp }}$, when the theoretical Casimir forces are computed using the dielectric permittivity of the generalized plasma-like model (14). As can be seen in Fig. 8 (b), this theoretical description of low-resistivity $\mathrm{Si}$ is consistent with the data within a $95 \%$ confidence interval. At the same time, more than $5 \%$ of grey dots lie outside the $95 \%$ confidence interval within the separation region from 62 to $200 \mathrm{~nm}$. This means that theoretical description of the second plate by means of the dielectric permittivity of high-resistivity $\mathrm{Si} \varepsilon_{\mathrm{ce}}^{(2)}(i \xi)$ is excluded by the data at a $95 \%$ confidence level.

As was noted above, successive measurements of the Casimir force in the configuration of an Au-coated sphere and two Si plates of different resistivities are not enough precise to discriminate between the descriptions of low-resitivity Si by means of Eqs. (14) and (15) (the generalized plasma-like and Drude-like models). There is a proposal 15 in the literature that this can be done by means of a patterned Si plate with two sections of different dopant concentrations. Such a plate is mounted on a piezoelectric actuator below an Au-coated sphere attached to the cantilever of an AFM (see Fig. 9). The actuator oscillates in the horizontal direction, and the cantilever flexes in response to the Casimir force above different regions of the plate. Thus, the sphere in such an experiment is subjected to the difference Casimir force which is an immediately measured quantity. In this case individual forces between the sphere and each of the two sections of the plate are not measured.

The patterned plate is composed of single-crystal Si specially fabricated to have adjacent sections with two different charge carrier densities. In so doing both $p$ - and $n$-type dopants can be used (B and $\mathrm{P}$, respectively). A sharp transition boundary between these sections with a width less than $200 \mathrm{~nm}$ can be achieved. Identically prepared but unpatterned samples can be used to measure the properties which are needed for the theoretical computations (with Hall probes for measuring the charge carrier density, and a four-probe technique for measuring the conductivity). During the measurements of the difference Casimir force, the distance between the sphere and the patterned plate is kept fixed and the plate oscillates in the horizontal direction, such that the sphere crosses the boundary between the two sections in the perpendicular direction during this oscillation. Note that a similar approach has been exploited $\sqrt[55]{5}$ for constraining new forces using the oscillations of an Au-coated sphere above two dissimilar metals. The Casimir force on the sphere changes as the sphere crosses the boundary. This change corresponds to the difference force $F_{\text {diff }}$, equal to the difference between the Casimir forces due to the different charge carrier densities $n_{a}$ and $n_{b}$ in different sections. Preliminary estimations show $\underline{45}$ that the 


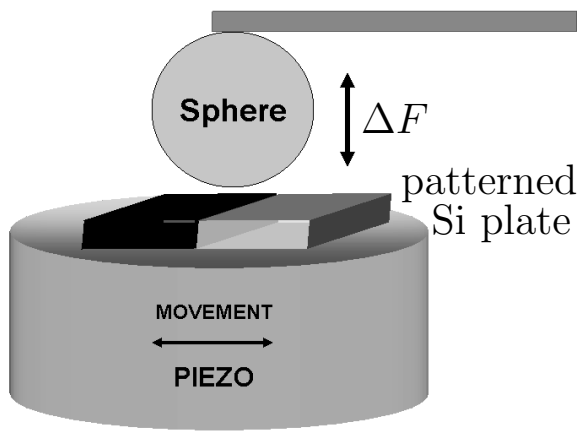

Fig. 9. Schematic diagram of an experimental setup for the measurement of the difference Casimir force between an Au-coated sphere and a patterned Si plate.

increased sensitivity of this experimental scheme should be sufficient to discriminate between the plasma-like and Drude-like models of dielectric response.

The above results again demonstrate that for the semiconductor of metallic type the magnitude of the Casimir force depends on the density of charge carriers which should be included in the model of the dielectric response in the Lifshitz theory. It is important also that the use of different semiconductor materials provides a way of controlling the magnitude of the Casimir force. One example was already provided in the end of Sec. 2.1. Here, for an Au sphere interacting with the first plate (highresistivity $\mathrm{Si}$ ), the magnitudes of the Casimir force are equal to $377.696,89.3329$, and $34.2372 \mathrm{pN}$ at separations $a=62.38,104.88$ and $147.38 \mathrm{~nm}$, respectively. This should be compared with the magnitudes of the Casimir force, $\left|F_{\mathrm{Au}-\mathrm{Au}}^{\text {theor }}\right|$, between the same $\mathrm{Au}$ sphere and $\mathrm{Au}$ plate $520.831,134.635$, and $54.7224 \mathrm{pN}$ at the same respective separations. From this one can conclude that the replacement of an $\mathrm{Au}$ plate with a high-resistivity $\mathrm{Si}$ one results in a decrease of the magnitude of the Casimir force by $27.5 \%, 33.6 \%$, and $37.4 \%$ at the respective separations indicated above. Smaller changes occur when an Au plate is replaced with the second Si plate (low-resistivity $\mathrm{Si}$ ). Here the force magnitudes are decreased by $22.6 \%, 26.4 \%$, and $28.0 \%$ at $a=62.38,104.88$ and $147.38 \mathrm{~nm}$, respectively.

\subsection{ITO plate}

One more demonstration that the magnitude of the Casimir force depends on the dielectric properties of metallic-type semiconductors has been performed $29 \mid 30$ in the configuration of an Au-coated sphere and an ITO plate $\left(\operatorname{In}_{2} \mathrm{O}_{3}: \mathrm{Sn}\right)$. Advantages of using ITO in the Casimir physics were proposed in Ref. 11. The experimental setup used an AFM schematically shown in Fig. 1 with a sphere of $R=100 \mu$ m radius. Main differences from experiments described above are that the measurements were performed in an ambient environment in the dynamic mode. This means that the 
AFM cantilever with the sphere attached to it was considered as a harmonic oscillator with a natural resonant frequency $\omega_{0}=2 \pi \times 1900 \mathrm{rad} / \mathrm{s}$. Under the influence of the Casimir force $F(a)$ the value of the resonant frequency was changed to some $\omega_{r}$. In the linear regime we get ${ }^{5}$

$$
\frac{\partial F}{\partial a}=k_{0}-k_{\mathrm{eff}}
$$

where $k_{0}=m \omega_{0}^{2}, k_{\text {eff }}=m \omega_{r}^{2}$, and $m$ is the mass of the oscillator.

Earlier the frequency shift $\omega_{0}-\omega_{r}$ from the dynamic scheme was measured directly using the frequency modulation technique in experiments on the determination of the Casimir pressure between two metallic surfaces by means of a micromechanical torsional oscillator. $17-20 \mid 56 / 57$ Note that the PFA allows recalculation of the gradient of the Casimir force between a sphere and a plate into the Casimir pressure between the two parallel plates according to $\underline{5 \mid 16}$

$$
P(a)=-\frac{1}{2 \pi R} \frac{\partial F}{\partial a} .
$$

In the experiment $\frac{29130}{2}$ the frequency modulation technique was not used. Instead, the oscillation amplitude at a fixed frequency was monitored. Then the resonant frequency shift due to the Casimir force led to a change in amplitude which can be related to the force gradient (similar dynamic scheme was used in the measurements 58 of the gradient of the Casimir force between metallic surfaces by means of an AFM, but the phase of the oscillator was monitored instead of the amplitude). However, it is necessary to be cautious with both amplitude and phase detection methods. The problem is that oscillator amplitude and phase can change not only because of frequency shift, but also because of change in dissipation (oscillator energy loss). Dissipation is of particular concern if the experiments are done in air as the air layer thickness between plate and sphere keeps changing when the plate is moved closer to the sphere.

Another feature of this experiment is that it consisted of the two measurements. In the first measurement the force between an $\mathrm{Au}$ coated sphere and an Au-coated sapphire plate was measured. In the second measurement this plate was replaced with a glass substrate coated with an ITO film of thickness of $190 \mathrm{~nm}$, resistivity $\rho \approx 1.6 \times 10^{-4} \Omega \mathrm{cm}$ and charge carrier density $n \approx 1.2 \times 10^{21} \mathrm{~cm}^{-3}$. Thus, the measure of reduction in the force magnitudes due to the replacement of Au with the semiconductor can be found with no comparison to the theoretical results.

The relative random error of the total force gradient measurements (electric plus Casimir) at a separation $95 \mathrm{~nm}$ was estimated 30 as about $3.5 \%$ of force gradient. Keeping in mind that the random errors in both total and electric forces are distributed normally, the relative random error in the measurements of the Casimir force cannot be smaller than $3.5 \%$. In addition, the measured Casimir force gradients were affected 30 by a $3 \%$ systematic error. The absolute random error of $0.5 \mathrm{~nm}$ in the determination of initial separation (chosen at about $8.5 \mu \mathrm{m}$ ) was reported 30 During the 580 measurement runs, the mechanical drift of initial separation for 
$52 \mathrm{~nm}$ was observed. The absolute systematic error in the initial separation was of about $1.4 \mathrm{~nm}$. From the comparison of the measured gradient of the Casimir force in $\mathrm{Au}-\mathrm{Au}$ and $\mathrm{Au}-\mathrm{ITO}$ configurations it was concluded that the replacement of an Au plate with an ITO plate leads to roughly $40 \%-50 \%$ smaller Casimir pressure between two parallel plates at separations from 80 to $120 \mathrm{~nm}$. Note that in an ambient environment used in these measurements the residual potential difference $V_{0}$ between the sphere and the plate varied with time significantly in the Au-ITO configuration (from 72 to $50 \mathrm{mV}$ at the separation of $100 \mathrm{~nm}$ ).

The comparison of the experimental data with theory was performed using the Lifshitz formula (8) at zero temperature and Eq. (10) with energy instead of the free energy, although measurements were performed at $T=300 \mathrm{~K}$. In this comparison the contribution of the surface roughness to the gradient of the Casimir force was disregarded. In fact an Au-coating on the polystyrene sphere was characterized 29130 by stochastic roughness with the variance $\delta=3.8 \mathrm{~nm}$. For the Au and ITO coatings on the plates $\delta=0.8$ and $4 \mathrm{~nm}$, respectively. Note that surface roughness increases the magnitude of the Casimir force. If corrections due to surface roughness were included in computations of the Casimir force, this would lead to about $2 \%$ correction in the case of Au-Au surfaces and $4 \%$ correction for Au-ITO surfaces at the separation $a=80 \mathrm{~nm}$. The dielectric permittivity of $\mathrm{Au}$ along the imaginary frequency axis was described by the tabulated optical data 51 for $2 n_{1}(\omega) n_{2}(\omega)$ (see Sec. 2.1) extrapolated to low frequencies by the imaginary part of the Drude function (13). For the dielectric permittivity of ITO Eq. (15) was used where the representation for $\varepsilon_{\mathrm{ce}}^{(2)}(i \xi)$ was taken from Ref. 59. As a result, different powers of separations for data and theory were obtained $\stackrel{30}{-}$ This can be explained by the use of the Lifshitz theory at zero temperature for the comparison with the room-temperature measurement data (for Au test bodies this approach was experimentally excluded $\frac{60}{6}$ at a $70 \%$ confidence level). Further refinement of the dielectric properties of ITO is also needed to bring the experimental data in agreement with theory.

To conclude, this measurement is another confirmation that using different semiconductor materials one can control the magnitude of the Casimir force.

\subsection{Ge spherical lens above a Ge plate}

All the experiments using semiconductor test bodies, which were discussed in Secs. 2.1-2.3, have been performed with the help of an AFM and spheres of about $100 \mu \mathrm{m}$ radius. In fact the cantilever of an AFM with attached sphere can be considered as a micromechanical device having a very high force sensitivity. This made the use of an AFM in measurements of the Casimir force, suggested for the first time for two metallic test bodies in Ref. 13, very productive. An alternative approach to measuring the Casimir force by using a torsion pendulum and a spherical lens of more that $10 \mathrm{~cm}$ curvature radius was suggested for two metallic test bodies in Ref. 61. This approach has an advantage in that it deals with large lenses of centimeter-size curvature radii and, thus, much larger forces allowing measurements 
at separations above $1 \mu \mathrm{m}$. Below it is shown, however, that the use of large lenses leads to serious problems regarding the reproducibility of measurement results and the comparison between experiment and theory.

Now we consider the measurement of the Casimir force between a crystalline intrinsic Ge plate and a crystalline intrinsic Ge lens of curvature radius $R=15.10 \pm$ $0.05 \mathrm{~cm}$ performed 31 by means of a torsion pendulum. The Ge lens was mounted on a piezoelectric $x y z$ motion stage and the Ge plate on one arm of the torsion pendulum with a body of $15 \mathrm{~cm}$ length in vacuum of $5 \times 10^{-7}$ Torr. A pendulum body was suspended by a tungsten wire of length $2.5 \mathrm{~cm}$ and diameter $25 \mu \mathrm{m}$. The other arm of the pendulum played the role of the center electrode situated in between two fixed compensator plates forming two parallel plate capacitors $C_{1}$ and $C_{2}$. The Casimir force between a Ge lens and Ge plate resulted in a torque which rotated the pendulum body. This resulted in changes in the capacitances $C_{1}$ and $C_{2}$, which were detected with a phase sensitive circuit. Then, compensating voltages were applied to the capacitances through a feedback circuit to counteract the change in the angle of the torsion pendulum and to keep the system in equilibrium. These compensating voltages were a measure of the Casimir force.

The calibration of the setup was performed by means of the measurements of electric forces. It was found, however, that the residual potential difference $V_{0}$ depends on separation where it was measured. According to Ref. 31, this might be explained by the polishing stresses or the curvature of the lens surface changing the crystal plane orientation at the surface. In addition, it was suggested that there was the contribution to electric force due to random short-scale patches. As a result, even after the application of the compensating potential, there was a residual electrostatic force of a complicated nature. An expression for this force containing three fitting parameters was obtained at large separations, where the Casimir force was assumed to be negligibly small. The values of the fitting parameters were determined from the fit to the experimental data of electric force measurements at large separations. Then, the obtained expression for the electric force was extrapolated to lower separation distances and subtracted from the experimental data for the total measured force at all separations in order to get the measured data for the Casimir force alone. This procedure seems to be not enough justified because, even if the found phenomenological expression for the electric force is applicable at large separations, it might incorrectly describe the residual electric force at short separations.

The obtained experimental Casimir forces were compared with five theoretical approaches. Within the first approach intrinsic Ge was considered as a highresistivity dielectric described by the dielectric permittivity of core electrons $\varepsilon_{\mathrm{ce}}^{(1)}(i \xi)=\varepsilon_{\mathrm{ce}}^{(2)}(i \xi) \equiv \varepsilon_{\mathrm{Ge}}(i \xi)$. In the second approach, the free charge carriers (electrons and holes) were taken into account by means of the Drude model us- 
ing Eq. (15), but with account of charge carriers of two types:

$$
\tilde{\varepsilon}^{(1)}(i \xi)=\tilde{\varepsilon}^{(2)}(i \xi)=\frac{\omega_{p, n}^{2}}{\xi\left(\xi+\gamma_{n}\right)}+\frac{\omega_{p, p}^{2}}{\xi\left(\xi+\gamma_{p}\right)}+\varepsilon_{\mathrm{Ge}}(i \xi) .
$$

Here, for intrinsic Ge we have $62 \omega_{p, n} \approx 7.8 \times 10^{11} \mathrm{rad} / \mathrm{s}, \omega_{p, p} \approx 5.9 \times 10^{11} \mathrm{rad} / \mathrm{s}$, and $\gamma_{n} \approx \gamma_{p} \approx 2.6 \times 10^{11} \mathrm{rad} / \mathrm{s}$. The respective charge carrier densities at $T=300 \mathrm{~K}$ are $n_{n}=n_{p} \approx 2.3 \times 10^{13} \mathrm{~cm}^{-3}$. In the framework of the third approach free charge carriers were taken into account by means of the plasma model similar to Eq. (14):

$$
\varepsilon^{(1)}(i \xi)=\varepsilon^{(2)}(i \xi)=\frac{\omega_{p, n}^{2}}{\xi^{2}}+\frac{\omega_{p, p}^{2}}{\xi^{2}}+\varepsilon_{\mathrm{Ge}}(i \xi) .
$$

Finally, as the fourth and fifth approaches, the modifications $37 / 38$ of the Lifshitz theory have been used in the comparison with the experimental data (these modifications were mentioned in Sec. 1 and are discussed in more detail in Sec. 3.4). In all cases computations of the theoretical Casimir force were performed using the PFA in Eq. (10).

The experimental data for the Casimir force were compared with theoretical results computed using the above five approaches and were found consistent with all of them within the limits of experimental errors. According to Ref. 31, "The error bars take into account all statistical uncertainties (2\%-3\%) as well as fitting uncertainties from the electrostatic force analysis (10\%)." The confidence level is not reported. However, these estimates of Ref. 31 do not correspond and match to those reported in rest of the paper. For example, using the given expression for the residual electrostatic force, 31 one obtains at separations of 1,2 , and $3 \mu \mathrm{m}$ that it is determined with the minimum absolute errors equal to 67,33 , and $22 \mathrm{pN}$, respectively. As the Casimir force is obtained from the subtraction of the residual electrostatic force from the total measured force, its error cannot be less than these at the corresponding distances. Thus, the correct relative errors of the Casimir force at 1,2 , and $3 \mu \mathrm{m}$ are no less than $40 \%, 124 \%$, and $211 \%$, respectively.

There is a discussion in the literature concerning the use of spherical $63-67$ and cylindrica $\sqrt[68]{ }$ metallic test bodies of centimeter-size radii of curvature for the measurements of the Casimir force. Problems emerged when an anomalous forcedistance relation for the electric force between an Au-coated spherical lens of $R=3.09 \mathrm{~cm}$ curvature radius and an Au-coated plate was observed, 63 distinct from that predicted by classical electrodynamics. As discussed above, anomalous electric forces of unclear origin emerged also between a Ge lens and Ge plate. It was shown 65 that the anomalous behavior of the electrostatic force can be explained due to deviations from a perfect spherical shape of the mechanically polished and ground surface for lenses with centimeter-size curvature radii (later this possibility

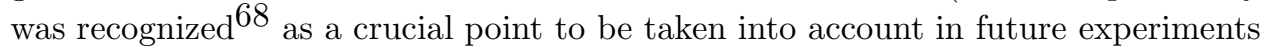
for a cylinder of centimeter size radius near a plate as well). Different kinds of surface imperfections (bubbles, pits and scretches) allowed by the optical surface 


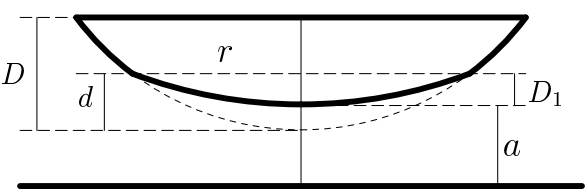

(a)

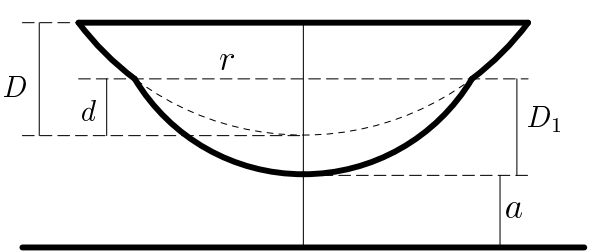

(b)

Fig. 10. The configuration of a spherical lens with radius of curvature $R$ possessing surface imperfection around the point of closest approach to a plate. (a) The bubble radius of curvature is $R_{1}>R$. (b) The bubble radius of curvature is $R_{1}<R$. The relative sizes of the lens and imperfection are not shown to scale.

specification data $29-74$ can lead to significant deviations of the force-distance relation from the form predicted by classical electrodynamics under an assumption of perfect spherical surface.

According to recent results, $\underline{6667}$ bubbles and pits, that are unavoidably present on spherical surfaces of centimeter-size curvature radii, make the PFA inapplicable in the form of Eq. (10) used to calculate the Casimir force in Ref. 31. As two of the simplest examples we consider a Ge lens of thickness $D$ with the curvature radius $R=15.1 \mathrm{~cm}$ having a bubble either of the radius of curvature $R_{1}=22 \mathrm{~cm}$ and thickness $D_{1}=0.09 \mu \mathrm{m}$ or $R_{1}=10 \mathrm{~cm}$ and $D_{1}=0.2 \mu \mathrm{m}$ near the point of the closest approach to a Ge plate [see Fig. 10(a) and (b), respectively]. The radii of the bubbles are determined from $r^{2}=2 R_{1} D_{1}-D_{1}^{2}$. This leads to bubble diameters $2 r=0.4 \mathrm{~mm}$ for the bubbles shown in Fig. 10(a,b). The obtained value should be compared with constraints imposed by the scratch/dig optical surface specifications of the used ${ }^{31}$ Ge lens of ISP optics 75 , GE-PX-25-50 with surface quality $60 / 40$. The latter means that $0.4 \mathrm{~mm}$ is just the maximum diameter of bubbles allowed. It is easily seen also that the flattening of the lens surface in Fig. 10(a) or the swelling up in Fig. 10(b) are much less than the absolute error of $R$ equal $\mathrm{td} \Delta R=0.05 \mathrm{~cm}$. Really, the quantity $d$ defined in Fig. 10(a,b) is equal to $d \approx r^{2} /(2 R) \approx 0.13 \mu \mathrm{m}$. This results in the flattening of the lens surface in Fig. 10(a) given by $d-D_{1} \approx 0.04 \mu \mathrm{m}$ or to the swelling up in Fig. 10(b) given by $D_{1}-d \approx 0.07 \mu \mathrm{m}$.

For a spherical lens with bubbles shown in Fig. 10(a,b) and a plate, the Casimir force is presented $\sqrt{6667}$ by the following generalization of the PFA:

$$
F(a, T)=2 \pi\left(R-R_{1}\right) \mathcal{F}\left(a+D_{1}, T\right)+2 \pi R_{1} \mathcal{F}(a, T) .
$$

Computations using the dielectric permittivity 76 of intrinsic Ge, $\varepsilon_{\mathrm{ce}}(i \xi)$, show that for the above parameters of the bubble in Fig. 10(a) Eq. (20) leads to the larger magnitudes of the Casimir force by $15 \%$ and $10 \%$ than Eq. (10) at separations $a=$ 0.6 and $1 \mu \mathrm{m}$, respectively. For the bubble of Fig. 10(b) the use of Eq. (20) instead of Eq. (10) results in smaller magnitudes of the Casimir forces by $19 \%$ and $14 \%$ at the same respective separations. Keeping in mind that experimentally it is hard to 
determine the point of the closest approach between the two surfaces with sufficient precision and to investigate the character of surface imperfection at this point, the possibility of using lenses of centimeter-size radius of curvature in measurements of the Casimir force becomes problematic. Specifically, it was shown 67 that the Casimir force between a perfectly spherical lens and a plate described by the Drude model can be made approximately equal to the force between a sphere with some surface imperfection and a plate described by the plasma model, and vice versa. This makes uncertain the results of such experiments as purported observation 77 of the thermal Casimir force using a spherical lens of $15.6 \mathrm{~cm}$ radius of curvature.

The problem of surface imperfections is not relevant to polystyrene spheres of about $100 \mu \mathrm{m}$ radii made by the solidification from the liquid phase. The surface quality of such spheres after metallic coating was investigated using a scanning electron microscope $5 \sqrt{5 / 15}$ and did not reveal any bubbles or scratches. Thus, in experiments discussed in Secs. 2.1-2.3 the PFA in its simplest form presented in Eq. (10) is applicable. It is applicable also in the proposed experiment ${ }^{78}$ using a cylinder-plate configuration with cylinders of about $100 \mu \mathrm{m}$ radii.

\section{Optically modulated Casimir force}

As discussed in Sec. 1, there are two experiments on the Casimir effect dealing with two macroscopic bodies which are inconsistent with the Lifshitz theory under some conditions. One of these experiments was performed three times $\frac{516-20}{-20}$ with metallic test bodies. It is outside the framework of our review. The other experiment $\underline{32 \mid 33}$ is a measurement of the optically modulated difference Casimir force between an $\mathrm{Au}$-coated sphere and a semiconductor ( $\mathrm{Si}$ ) plate illuminated with laser pulses. It is considered in more detail in this section. The experiment on the optically modulated Casimir force is important in two aspects. On the one hand, it provides new fundamental insights by demonstrating that the Lifshitz theory and its modifications are incompartible with the inclusion of dc conductivity of dielectric materials in the model of the dielectric response. On the other hand, technologically the optical modulation experiment opens opportunities to periodically change the magnitude of the Casimir force and to realize the pulsating regime without use of mechanical springs. This is discussed in Sec. 6.

\subsection{Experimental scheme and measurements results}

The experiment ${ }^{32} \mid 33$ on the optical modulation of the Casimir force is a direct measurement of the change in the force between an Au-coated sphere of $197.8 \pm$ $0.3 \mu \mathrm{m}$ diameter and a Si plate in the presence and in the absence of a laser light on it. Being a difference measurement (the Casimir forces with and without light on the plate are not measured separately but only their difference), this experiment is characterized by a very high force sensitivity of a few tens of $\mathrm{pN}$. The experimental scheme is shown in Fig. 11. Measurements were made by means of an AFM in an oil-free vacuum chamber with a pressure of around $2 \times 10^{-7}$ Torr. The Si plate 


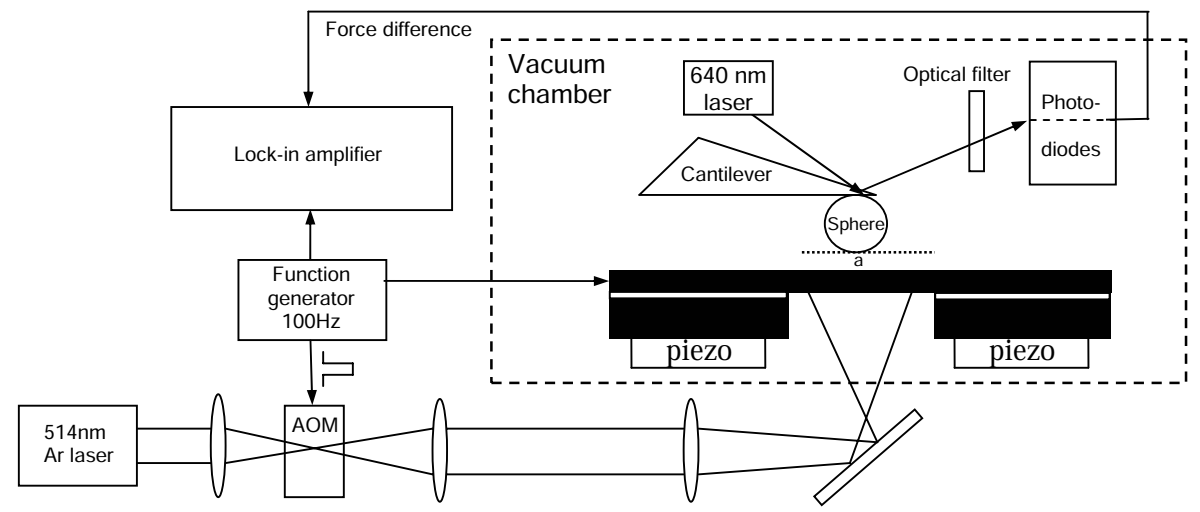

Fig. 11. Schematic of the experimental setup for studying optical modulation of the Casimir force. The main components are shown.

was mounted on the top of a piezoelectric actuator, which was used to change the separation $a$ between the sphere and the plate from contact to $6 \mu \mathrm{m}$. The Si used was $p$-type with charge carrier density equal to $n \approx 5 \times 10^{14} \mathrm{~cm}^{-3}$. The excitation of additional carriers in the Si plate was done with $5 \mathrm{~ms}$ wide light pulses obtained from Ar laser at $514 \mathrm{~nm}$ wavelength, modulated at a frequency of $100 \mathrm{~Hz}$. The laser pulses were focused on the bottom surface of the Si plate (see Fig. 111).

The cantilever of the AFM flexed when the Casimir force between the sphere and the plate changed depending on the presence or absence of laser light on the plate. Similar to Fig. 1, the cantilever deflection was monitored with a $640 \mathrm{~nm}$ beam from a second laser shown in Fig. 11. An optical fiber was used to prevent interference of the beams from both lasers. The change in the Casimir force due to the light incident on the plate led to a difference signal between the two photodiodes (see Fig. 11). This signal was measured with a lock-in amplifier.

The most important part of the described setup is the Si plate colored black in Fig. 11. It should be sufficiently thin and of appropriate resistivity to ensure that the density of charge carriers increases by several orders of magnitude under the influence of laser pulses (note that the charge carrier density indicated above corresponds 51 to relatively high resistivity $\rho=10 \Omega \mathrm{cm}$ ). At the same time Si plate should be thick enough to make as low as possible the photon pressure of the transmitted light on the sphere. The Si plate shown in Fig. 11 was fabricated in several steps. As an initial sample, the commercial wafer of Si grown on an insulator $\left(\mathrm{SiO}_{2}\right)$ was used. The wafer consisted of a Si substrate of thickness $600 \mu \mathrm{m}$ and a Si top layer of thickness $5 \mu \mathrm{m}$ with a buried intermediate $\mathrm{SiO}_{2}$ of thickness $400 \mathrm{~nm}$ [see Fig. 12(a) where $\mathrm{Si}$ is colored black and $\mathrm{SiO}_{2}$ white]. The thickness of the $\mathrm{Si}$ substrate was reduced to about $200 \mu \mathrm{m}$ and then, after RCA cleaning of the surface, the wafer was oxidized at high temperature in a dry $\mathrm{O}_{2}$ atmosphere. As a result, a 
(a)

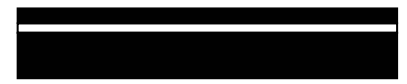

(b)

(c)
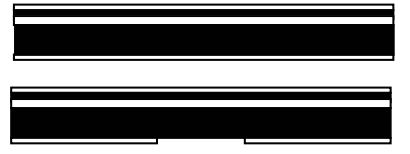

(d)

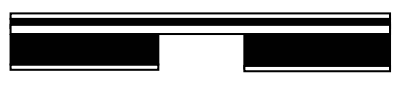

(e)

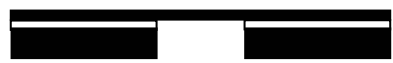

Fig. 12. Fabrication process of the Si membrane. (a) The Si substrate (colored black) with a buried $\mathrm{SiO}_{2}$ layer (white). (b) The substrate was mechanically polished and oxidized, and (c) a window in the bottom $\mathrm{SiO}_{2}$ layer was etched with HF. (d) Next, TMAH was used to etch the $\mathrm{Si}$. (e) Finally, the $\mathrm{SiO}_{2}$ layer was etched away in $\mathrm{HF}$ solution to form a clean $\mathrm{Si}$ surface.

thermal oxide layer with a thickness of about $1 \mu \mathrm{m}$ was formed on the bottom and top sides of the wafer [see Fig. 12(b)]. Then a hole with a diameter of $0.85 \mathrm{~mm}$ was etched with HF in the center of the bottom oxide layer [Fig. 12(c)]. Next, TMAN was used at $363 \mathrm{~K}$ to etch the $\mathrm{Si}$ substrate (note that TMAN etching rate for Si is 1000 times greater than for $\mathrm{SiO}_{2}$ ). As a result, a hole was formed as shown in Fig. 12(d). Finally, all the thermal oxidation layers and the buried oxidation layer in the hole were etched away in HF solution to form a clean Si plate (membrane) over a hole [see Fig. 12(e)]. The thickness of this plate was measured to be $d=4.0 \pm 0.3 \mu \mathrm{m}$.

All calibrations were done by applying different potentials to the Si plate while keeping the sphere grounded. The calibrations were made in a similar way to other experiments with semiconductor surfaces. As a result the signal calibration constant and the residual potential differences $V_{0}=-0.225 \pm 0.002 \mathrm{~V}$ and $V_{0}^{l}=-0.303 \pm$ $0.002 \mathrm{~V}$ in the absence and in the presence of the laser light on the Si plate were determined. The residual potential differences were shown to be independent of separation (all details of calibration procedures can be found in Refs. 5, 33 and 54).

The measurement of the difference Casimir force in the presence and in the absence of a light pulse on a Si plate as a function of separation was performed as follows. During the bright phases of the pulse train, a voltage $V^{l}$ was applied to the plate, and during the dark phases, a voltage $V$. Then the difference of the total force, $F_{\text {diff }}^{\text {tot }}(a)$ (Casimir and electric), was measured. This was done using a lock-in amplifier with an integration time of $100 \mathrm{~ms}$, which corresponds to a bandwidth of $0.78 \mathrm{~Hz}$. The difference between the Casimir forces in the presence and in the absence of light,

$$
F_{\text {diff }}^{\text {expt }}(a)=F_{l}(a)-F(a),
$$


was found by subtracting the contribution of the electric forces:

$$
F_{\mathrm{diff}}^{\text {expt }}(a)=F_{\mathrm{diff}}^{\mathrm{tot}}(a)-X\left(\frac{a}{R}\right)\left[\left(V^{l}-V_{0}^{l}\right)^{2}-\left(V-V_{0}\right)^{2}\right],
$$

where $X(z)$ is the function whose explicit form is known from the exact solution of the electrostatic problem in the sphere-plate geometry. $[5|16| 27 / 33 \mid 79$ The difference Casimir forces as a function of separation were found for different absorbed laser powers: $P^{\mathrm{eff}}=9.3,8.5$, and $4.7 \mathrm{~mW}$. At each absorbed power measurements were repeated with different pairs of applied voltages $\left(V^{l}, V\right)$ between the sphere and the plate in the bright and dark phases, respectively, and the mean difference Casimir force, $\bar{F}_{\text {diff }}^{\text {expt }}$, was obtained. Below we consider the experimental data for the maximum, $P^{\text {eff }}=9.3 \mathrm{~mW}$, and minimum, $4.7 \mathrm{~mW}$, absorbed powers averaged over 31 and 33 repititions with different pairs of applied voltages. With these absorbed laser powers, charge carrier densities in $\mathrm{Si}$ in the bright phase were equal to $n_{l}=(2.1 \pm 0.4) \times 10^{19} \mathrm{~cm}^{-3}$ and $(1.4 \pm 0.3) \times 10^{19} \mathrm{~cm}^{-3}$, respectively, where

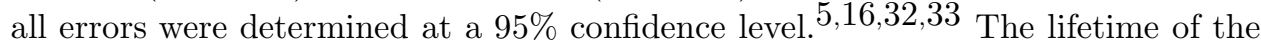
charge carriers excited in the Si plate by the pulses from the Ar laser was measured using a noninvasive optical pump-probe technique. $80[81$ This time represents both surface and bulk recombination and is consistent with that expected for Si. The measured values of the carrier lifetime were used in theoretical computations of the change in the Casimir force for different incident laser powers.

In Fig. 13 $(a, b)$ the experimental data for $\bar{F}_{\text {diff }}^{\text {expt }}(a)$ as a function of separation are shown by dots for absorbed powers $P^{\text {eff }}=9.3$ and $4.7 \mathrm{~mW}$, respectively. The corresponding incident powers were 15.0 and $7.6 \mathrm{~mW}$. As expected, the magnitude of the Casimir force difference has the largest values at the shortest separations and decreases with the increase of separation. Some oscillations of the data with the distance in Fig. 13(a,b) come from the interference of one beam of light of $640 \mathrm{~nm}$ laser scattered by the plate with another beam scattered from the sphere. Below we present the results of error analysis and the comparison of the experimental data with theory.

\subsection{Analysis of errors and uncertainties}

Here, we discuss both the experimental and theoretical errors arising in measurements and computations of the difference Casimir forces. The main role in the experimental error in this experiment is played by the random error. The absolute random error in this experiment as a function of separation was found $32 \mid 33$ by the standard procedure using Student's $t$-distribution with the number of degrees of freedom 30 and 32 for the measurements with two different absorbed powers. In so doing the $95 \%$ confidence level was chosen. The obtained random errors decrease from 0.65 to $0.29 \mathrm{pN}$ and from 0.32 to $0.24 \mathrm{pN}$ for the absorbed powers 9.3 and $4.7 \mathrm{~mW}$, respectively, when the separation increases from 100 to $250 \mathrm{~nm}$. The main systematic errors were from the instrumental noise, $\Delta_{1}^{\text {syst }} F_{\text {diff }}^{\text {expt }} \approx 0.08 \mathrm{pN}$, from the resolution error in data acquisation, $\Delta_{2}^{\text {syst }} F_{\text {diff }}^{\text {ext }} \approx 0.02 \mathrm{pN}$, and from calibration, 

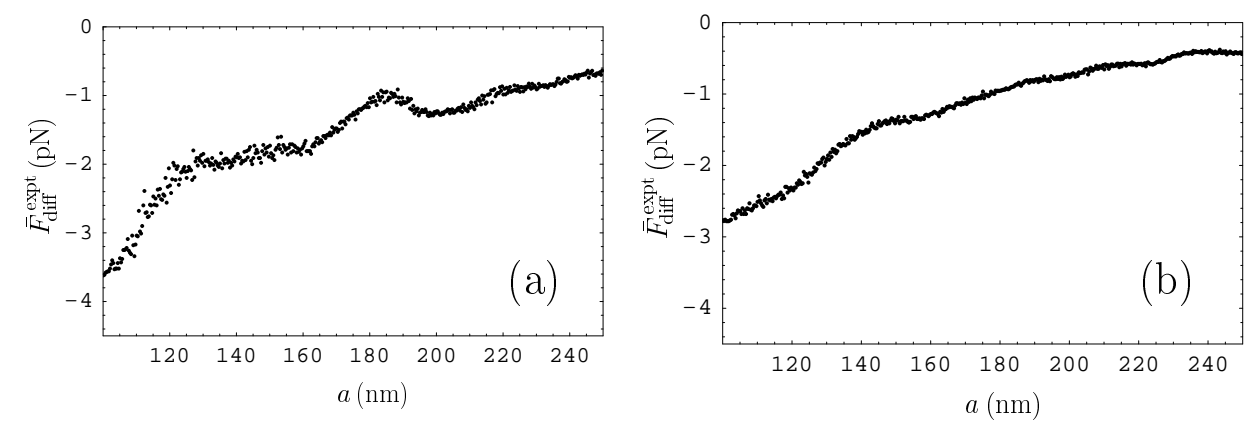

Fig. 13. Mean measured differences of the Casimir force between an Au-coated sphere and Si plate in the presence and in the absence of light for the absorbed power of (a) $9.3 \mathrm{~mW}$ and (b) $4.7 \mathrm{~mW}$ versus separation are shown by dots.
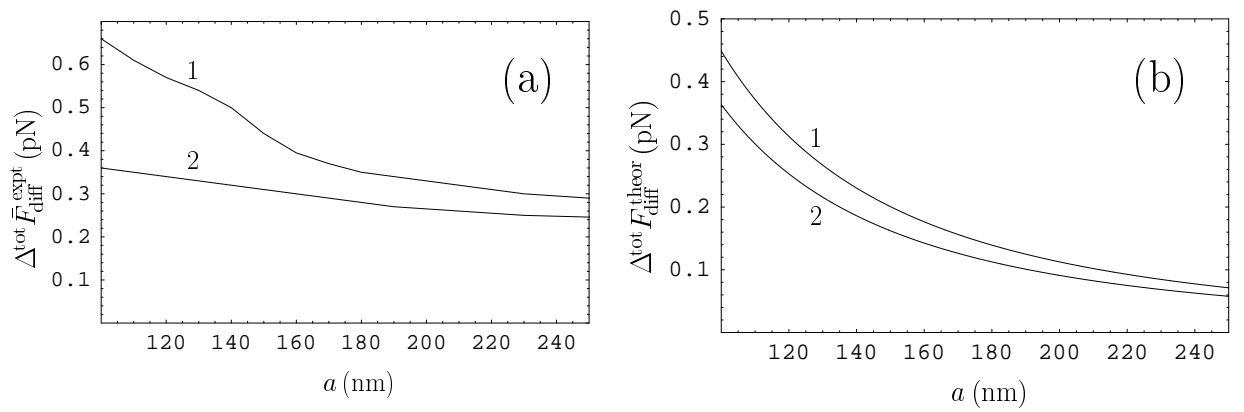

Fig. 14. (a) Total experimental errors and (b) total theoretical errors, versus separation. The cases of different absorbed powers 9.3 and $4.7 \mathrm{~mW}$ are labeled 1 and 2, respectively.

$\Delta_{3}^{\text {syst }} F_{\text {diff }}^{\text {expt }}$. The latter was equal to $0.6 \%$ of the measured difference Casimir force, whereas the two former were independent on separation. The three systematic errors were combined at a $95 \%$ confidence level by using the standard rule valid for quantities distributed uniformly (this is the most conservative approach $5[16 \mid 82$ ). As a result, the total systematic error varied from 0.092 to $0.095 \mathrm{pN}$ for measurements with different absorbed powers. The total experimental error determined by the random error was found at a $95 \%$ confidence level. For this purpose the statistical rule $\longdiv { 5 1 6 1 8 2 }$ adapted for the case when the quantities to be combined are described by the normal (or Student) and uniform distributions was used. In Fig. 14(a) the total experimental error as a function of separation is shown by the lines 1 and 2 for the absorbed powers 9.3 and $4.7 \mathrm{~mW}$, respectively. As can be seen in Fig. 14(a), the relative total experimental error varies from $10 \%$ to $20 \%$ at a separation $a=100 \mathrm{~nm}$ and from $25 \%$ to $33 \%$ at a separation $a=180 \mathrm{~nm}$ for different absorbed powers. The absolute error in the measurements of separations was $\Delta a=1 \mathrm{~nm}$.

Now we discuss the theoretical errors. The main source of the theoretical uncer- 
tainty in this experiment is the error in the concentration of charge carriers $n_{l}$ when the light is on. From Sec. 3.1, this error is of about 20\%. This leads to respective errors in the values of the plasma frequency (see Sec. 3.3) and results in the relative error in computed difference Casimir forces, $\delta_{1} F_{\text {diff }}^{\text {theor }}$, approximately equal to $12 \%$. This error does not depend on separation. Another theoretical error is due to the uncertainty of the experimental separations $a_{i}$ at which the values of the theoretical force $F_{\text {diff }}^{\text {theor }}$ should be computed. For each of the forces in the absence, $F^{\text {theor }}\left(a_{i}\right)$, and in the presence, $F_{l}^{\text {theor }}\left(a_{i}\right)$, of light this relative error is equal to $3 \Delta a_{i} / a_{i}$ and takes its maximum value $3 \%$ of the Casimir force at $a=100 \mathrm{~nm}$. This leads to only $\delta_{2} F_{\text {diff }}^{\text {theor }}=2 \%$ error in the difference Casimir force at $a=100 \mathrm{~nm}$ and to smaller errors at larger separations. Note that the other theoretical errors, such as due to sample-to-sample variations of the optical data, patch potentials, and the use of the PFA are negligibly small in comparison with the two errors listed above. The two errors $\delta_{1} F_{\text {diff }}^{\text {theor }}$ and $\delta_{2} F_{\text {diff }}^{\text {theor }}$ were combined at a $95 \%$ confidence level using the statistical rule for the combination of the quantities distributed uniformly $[5 / 16 / 27 / 33 \mid 82$ The resulting absolute total theoretical errors are shown as functions of separation in Fig. 14(b) by the lines 1 and 2 for the maximum and minimum absorbed powers, respectively. When calculating the absolute errors shown in Fig. 14(b) we used the Drude-like behavior of the dielectric permittivity at low frequencies because the results obtained are applied below to demonstrate an inconsistency between the Lifshitz theory and the Drude model approach. The use of the plasma-like behavior results in slightly larger absolute errors. The absolute total theoretical errors shown in Fig. 14(b) can be employed for the determination of the confidence interval of the quantity $F_{\text {diff }}^{\text {theor }}\left(a_{i}\right)-F_{\text {diff }}^{\text {expt }}\left(a_{i}\right)$ similar to those shown in Figs. 6 and 8 , It should be kept in mind, however, that the theoretical error $\delta_{2} F_{\text {diff }}^{\text {theor }}$ is not applicable to the widths of the theoretical bands computed over the entire measurement range, but only at each experimental point (see below Figs. 16 in Sec. 3.3 and 18 in Sec. 3.4).

In the original publications 3233 one more major theoretical error was considered connected with the pressure of light transmitted through the plate and incident on the bottom of the sphere. This effect is present only during the bright phase of the pulse train and at a separation $a=100 \mathrm{~nm}$ led to $2.3 \%$ and $1.5 \%$ errors in the difference Casimir force for the two absorbed powers. At $a=200 \mathrm{~nm}$ the relative total theoretical error due to light pressure was estimated as $8.9 \%$ and $5 \%$ for different absorbed powers $[53$ The repulsive force on the sphere due to the light pressure can be, however, computed and subtracted from the measured difference Casimir force at all experimental separations. In this way the corrected values of the difference Casimir force are restored which would be measured in the case when all $100 \%$ of the light from a $514 \mathrm{~nm}$ laser were absorbed in the Si plate. The magnitudes of corrected values are a bit larger than the original ones. Such a procedure in metrology is called a correction .82 Below we correct the experimental data for the difference Casimir force due to the presence of the light pressure, instead of including its role in the theoretical error.

If $I$ is the intensity of light incident on an area element $d S$ at an angle $\theta$, the 
magnitude of respective force due to the light pressure acting perpendicular to the plate takes the form

$$
\left|d \boldsymbol{F}_{\mathrm{lp}}\right|=2 \frac{I}{c} \cos \theta d S
$$

Let the $z$ axis be perpendicular to the plate and passes through the center of the sphere. The intensity of laser light incident on the spherical ring of radius $\rho=R \sin \theta$ and width $R d \theta$ on the soarce side of the plate is given by

$$
I(\rho)=\frac{2 \alpha_{0} P^{\mathrm{eff}}}{\pi w^{2}} e^{-2 \rho^{2} / w^{2}},
$$

where $\alpha_{0}$ is the fraction of the absorbed power transmitted through the plate and $w=0.23 \pm 0.01 \mathrm{~mm}$ is the Gaussian width of the focused beam on the plate. ${ }^{32}$ The value of $\alpha_{0}$ can be calculated as

$$
\alpha_{0}=r_{\mathrm{tr}} e^{-d / l_{\mathrm{opt}}} \approx 0.00641 .
$$

It is obtained using the transmission coefficient $r_{\mathrm{tr}} \approx 0.35$.

The area of a spherical ring of radius $\rho$ on a sphere surface set at an angle $\theta$ to the $z$ axis is equal to

$$
d S=2 \pi \rho R d \theta=2 \pi R^{2} \sin \theta d \theta .
$$

Then the force, $F_{\text {lp }}$, acting on a sphere owing to the light incident on the source side of the plate (i.e., in the $z$ direction) is the following:

$$
F_{\mathrm{lp}}=\int_{0}^{\pi / 2} d F_{\mathrm{lp}, z}(\theta)=\int_{0}^{\pi / 2} \cos \theta\left|d \boldsymbol{F}_{\mathrm{lp}}(\theta)\right|
$$

where $\left|d \boldsymbol{F}_{\text {lp }}(\theta)\right|$ is given by Eqs. (23)-(26). Introducing the new variable $t=\cos \theta$, Eq. (27) can be rearranged to the form

$$
F_{\mathrm{lp}}=\frac{8 \alpha_{0} R^{2} P^{\mathrm{eff}}}{c w^{2}} e^{-2 R^{2} / w^{2}} \int_{0}^{1} t^{2} e^{2 R^{2} t^{2} / w^{2}} d t .
$$

The last expression can be rewritten in terms of the imaginary error function $\operatorname{Erfi}(z)$ :

$$
F_{\mathrm{lp}}=\frac{2 \alpha_{0} P^{\mathrm{eff}}}{c}\left[1-f\left(\frac{\sqrt{2} R}{w}\right)\right],
$$

where

$$
f(x)=e^{-x^{2}} \frac{\sqrt{\pi} \operatorname{Erfi}(x)}{2 x} .
$$

Now we determine the error in calculation of the force $F_{\text {lp }}$ using Eqs. (29) and (30). The error in the quantity $f(x)$ in Eq. (30) is determined by the systematic errors in the measurements of sphere radius $R$ and the width of the beam $w(\delta R=$ $0.15 \%$ and $\delta w=4 \%$, respectively). Using the conservative assumption that these quantities take any value around their mean values in the limit of errors with equal probability (any other assumption leads to smaller error) we find the error of $x=$ 

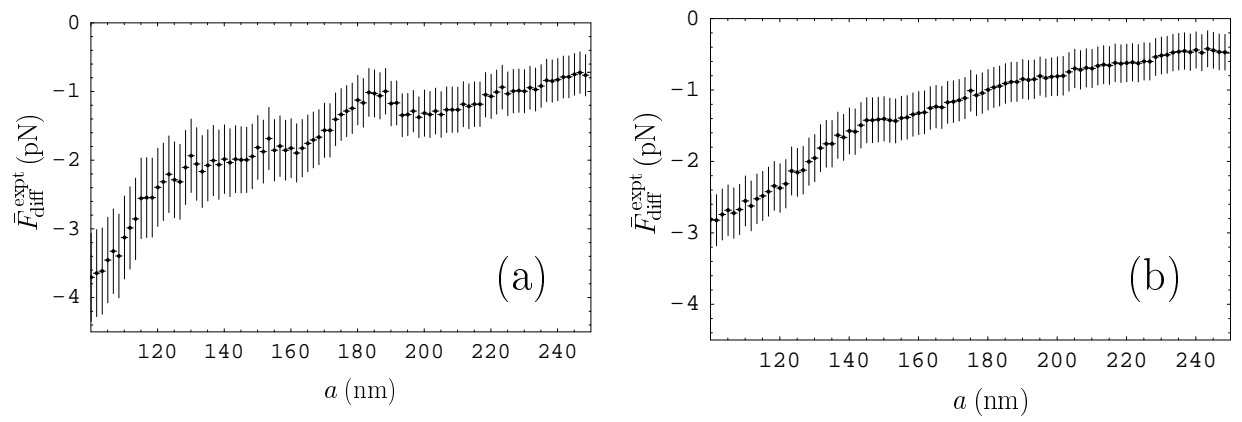

Fig. 15. (a) The experimental differences in the Casimir force in the presence and in the absence of light corrected for the light pressure are shown as crosses versus separation. The arms of the crosses are determined at a $95 \%$ confidence level. The absorbed powers are (a) $9.3 \mathrm{~mW}$ and (b) $4.7 \mathrm{~mW}$.

$\sqrt{2} R / w$ determined at a $95 \%$ confidence level, $\delta x \approx 0.04$, from the same combination rule $5 \sqrt{16 / 27 / 33 / 82}$ as was used above. Then from Eq. (30) one obtains $1-f(x)=$ $0.214 \pm 0.015$. The error of the factor $2 \alpha_{0} P^{\text {eff }} / c$ in Eq. (29) is mostly determined by the error of $\alpha_{0}$ defined in Eq. (25). The latter, in its turn, is determined by $\delta d \approx 7.5 \%$. From Eq. (25) this leads to $\alpha_{0}=r_{\text {tr }}(0.019 \pm 0.005)$, i.e., to $\delta \alpha_{0}=25 \%$. Combining the two errors with the help of the same combination rule, one obtains $\delta F_{\mathrm{p}}=28 \%$. This leads to an additional small systematic error in the corrected measured difference Casimir force. For example, at $a=100 \mathrm{~nm}$ the new systematic error is equal to $0.64 \%$ and $0.42 \%$ of the difference Casimir force for the two absorbed powers, i.e., of the same order as other systematic errors considered in Sec. 3.2. Although this results in a slightly different combined systematic error, the total experimental error shown in Fig.14(a) remains primarily determined by the random error and, thus, is pretty much unchanged.

In Fig. 15(a,b), the experimental data for the difference Casimir force for the absorbed powers of 9.2 and $4.7 \mathrm{~mW}$, respectively, with the subtracted force (29) due to the light pressure are shown as crosses. Here, the vertical arms of the crosses are equal to $2 \Delta^{\text {tot }} \bar{F}_{\text {diff }}^{\text {expt }}$ determined at a $95 \%$ confidence level [see Fig. 14(a)]. The horizontal arms of each cross are equal tp $2 \Delta a=2 \mathrm{~nm}$. This should be compared with Fig. 13 $(a, b)$ where the uncorrected data for the difference Casimir force are presented. To make Fig. 15(a,b) readable, we have plotted only each fifth dot shown in Fig. 13(a,b). In the following two sections, the experimental data of Fig. 15 are compared with computations using different theoretical approaches.

\subsection{Comparison with the Lifshitz theory}

Now we compare the experimental data for the difference in the Casimir forces between an Au-coated sphere and Si plate in the presence and in the absence of light on the plate with theoretical predictions of the Lifshitz theory. Computations of 
the Casimir force $F(a)=F(a, T)$ were performed at $T=300 \mathrm{~K}$ using the Lifshitz formula (3) and the PFA (10). For the dielectric permittivity of Au either the generalized plasma-like model (11) or the tabulated optical data ${ }^{51}$ extrapolated to low frequencies by the imaginary part of the Drude model (13) have been used. For Si in the absence of light the dielectric permittivity along the imaginary frequency axis, $\varepsilon_{\mathrm{ce}}^{(2)}(i \xi)$, is determined by the contribution of the core electrons (see Sec. 2.1). For high-resistivity, dielectric materials the role of dc conductivity was usually neglected (see, for instance, computations of the Casimir-Polder force between Rb atoms and $\mathrm{SiO}_{2}$ plate $e^{83}$. If, however, we would like to take into consideration the dc conductivity of the Si plate in the absence of light, the dielectric permittivity along the imaginary frequency axis takes the form 151

$$
\varepsilon^{(2)}(i \xi)=\frac{4 \pi \sigma_{0}^{(2)}(T)}{\xi}+\varepsilon_{\mathrm{ce}}^{(2)}(i \xi),
$$

where $\sigma_{0}^{(2)}(T)$ is the static conductivity. As was mentioned in Sec. 1, the substitution of the dielectric permittivity (31) into the Lifshitz theory results in the violation of the Nernst heat theorem. $34-36$

Both the violation of the Nernst theorem and significantly larger magnitudes of the Casimir force obtained when the dc conductivity is taken into account are explained by different contributions from the TM reflection coefficient (4) into the zero-frequency term of the Lifshitz formula (3). Thus, from Eqs. (3) and (10), for a metal sphere above a dielectric plate with dc conductivity neglected [Si is described by $\varepsilon_{\mathrm{ce}}^{(2)}$ and $\mathrm{Au}$ either by the generalized plasma-like model (11) or by the tabulated optical data extrapolated by the Drude model] the contribution of the zero-frequency term to the force is:

$$
F_{0}(a, T)=-\frac{k_{B} T R}{8 a^{2}} \operatorname{Li}_{3}\left[\frac{\varepsilon_{0}^{(2)}-1}{\varepsilon_{0}^{(2)}+1}\right] .
$$

Here $\varepsilon_{0}^{(2)} \equiv \varepsilon_{\mathrm{ce}}^{(2)}(0)$ and $\operatorname{Li}_{n}(z)$ is the polylogarithm function. Note that Eq. (32) represents the contribution of the TM mode alone because $r_{\mathrm{TE}}^{(2)}\left(0, k_{\perp}\right)=0$. If the dc conductivity of a dielectric plate is taken into account, i.e., the dielectric permittivity $\varepsilon^{(2)}(i \xi)$ in Eq. (31) is used instead of $\varepsilon_{\mathrm{ce}}^{(2)}(i \xi)$, the contribution of the zero-frequency term to the force is given by

$$
\tilde{F}_{0}(a, T)=-\frac{k_{B} T R}{8 a^{2}} \zeta(3)
$$

where $\zeta(z)$ is the Riemann zeta function. In so doing the contribution from the TE mode at zero frequency remains equal to zero. It is easily seen that $\left|\tilde{F}_{0}(a, T)\right|>$ $\left|F_{0}(a, T)\right|$. The measurements of the optically modulated Casimir force were of sufficient precision to check the predictions of the Lifshitz theory with dc conductivity included experimentally. 

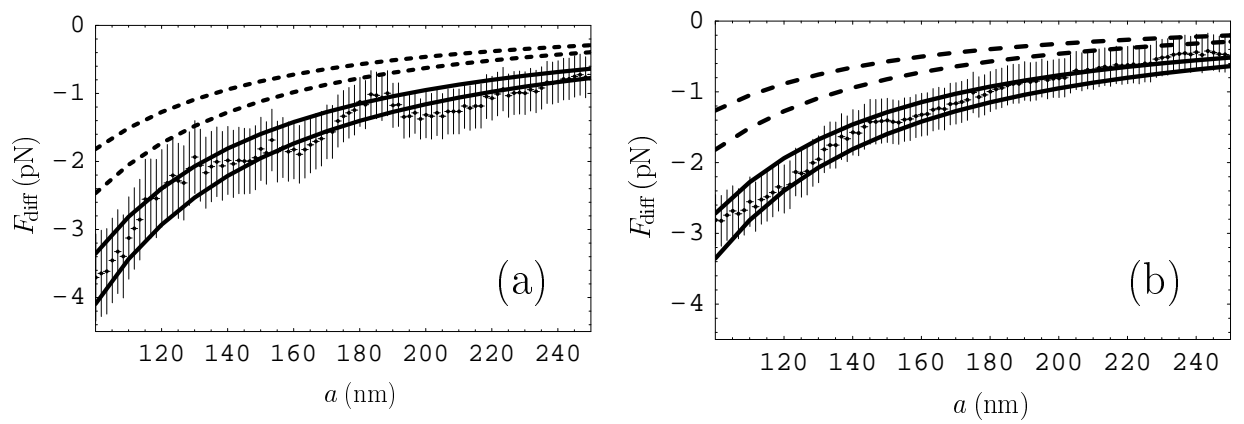

Fig. 16. The experimental differences in the Casimir force (crosses plotted at a $95 \%$ confidence level) are reproduced from Fig. 15 The theoretical bands confined between the pairs of solid lines and dashed lines are found at a 95\% confidence level with the dc conductivity of a Si plate in the dark phase disregarded and included, respectively. The absorbed powers are (a) $9.3 \mathrm{~mW}$ and (b) $4.7 \mathrm{~mW}$.

For $\mathrm{Si}$ in the presence of light the dielectric permittivity along the imaginary frequency axis was described using either the generalized Drude-like model

$$
\tilde{\varepsilon}_{l}^{(2)}(i \xi)=\frac{\omega_{p, n}^{2}}{\xi\left(\xi+\gamma_{n}\right)}+\frac{\omega_{p, p}^{2}}{\xi\left(\xi+\gamma_{p}\right)}+\varepsilon_{\mathrm{ce}}^{(2)}(i \xi)
$$

or the generalized plasma-like model

$$
\varepsilon_{l}^{(2)}(i \xi)=\frac{\omega_{p, n}^{2}}{\xi^{2}}+\frac{\omega_{p, p}^{2}}{\xi^{2}}+\varepsilon_{\mathrm{ce}}^{(2)}(i \xi) .
$$

Here, the values of the plasma frequencies obtained from the densities of charge carriers indicated above are the following: $\omega_{p, n}=(5.1 \pm 0.5) \times 10^{14} \mathrm{rad} / \mathrm{s}, \omega_{p, p}=$ $(5.7 \pm 0.6) \times 10^{14} \mathrm{rad} / \mathrm{s}$ for the absorbed power $P^{\mathrm{eff}}=9.3 \mathrm{~mW}$ and $\omega_{p, n}=(4.1 \pm$ $0.4) \times 10^{14} \mathrm{rad} / \mathrm{s}, \omega_{p, p}=(4.6 \pm 0.4) \times 10^{14} \mathrm{rad} / \mathrm{s}$ for the absorbed power $P^{\text {eff }}=$ $4.7 \mathrm{~mW}$. The values of the relaxation parameters were $\gamma_{n} \approx 1.8 \times 10^{13} \mathrm{rad} / \mathrm{s}$ and $\gamma_{p} \approx 5.0 \times 10^{12} \mathrm{rad} / \mathrm{s}$. They do not depend on the absorbed power.

The calculated differences of the Casimir force in the presence and in the absence of light, (21), were corrected for the presence of surface roughness using the nonmultiplicative approach of geometrical averaging, as discussed in Sec. 2.1. The force differences with inclusion of surface roughness are notated as $F_{\text {diff }}^{\text {theor }}(a)$. In the experiment on optically modulated Casimir force the contribution from the roughness correction was very small. Thus, at $a=100 \mathrm{~nm}$, it contributed only $1.2 \%$ of the calculated $F_{\text {diff }}^{\text {theor }}(a)$. At $a=150 \mathrm{~nm}$, the contribution from the surface roughness decreased to only $0.5 \%$ of the calculated force difference.

The comparisons between the experimental data and the Lifshitz theory for the absorbed powers of 9.3 and $4.7 \mathrm{~mW}$ are presented in Fig. 16(a,b), respectively. The experimental data for the difference in the Casimir forces in the presence and in the absence of light are shown as crosses. These crosses, whose arms are determined at a 95\% confidence level, are the same as in Fig. 15 (each fifth experimental data point 
is shown). The theoretical bands confined between the two solid lines are computed using the generalized plasma-like model for Au. For Si the dielectric permittivity of core electrons $\varepsilon_{\mathrm{ce}}^{(2)}(i \xi)$ was used in the dark phase and the generalized plasma-like model (35) in the bright phase. Note that the use of the tabulated optical data extrapolated to low frequencies by the Drude model for $\mathrm{Au}$ and the generalized Drude-like model for $\mathrm{Si}$ in the bright phase is illustrated in the next figure. The widths of the bands are determined by the errors in the plasma frequency of $\mathrm{Si}$ in the bright phase originating from the errors in the concentration of charge carriers. These bands are calculated not at the experimental separations, but over the entire measurement range. Because of this, their widths do not include uncertainties due to the experimental error $\Delta a$ in the measurement of separations. As can be seen in Fig. 16(a,b), both theoretical bands confined between the pairs of two solid lines are consistent with the data.

The theoretical bands confined between the two dashed lines in Fig. 16(a,b) are computed accounting for dc conductivity of a $\mathrm{Si}$ plate in the absence of light by means of Eq. (31). In accordance with this Si plate in the bright phase is described by the generalized Drude-like model (34) and Au by the tabulated optical data extrapolated by the Drude model to low frequencies. The widths of the dashed bands are again determined by large errors of the plasma frequencies of Si in the bright phase. As can be seen in Fig. 16(a,b), the Lifshitz theory taking into account dc conductivity of high-resistivity $\mathrm{Si}$ in the dark phase is experimentally excluded by the data over the entire range of separations from 100 to $250 \mathrm{~nm}$ (for the absorbed power $9.3 \mathrm{~mW}$ ) and from 100 to $230 \mathrm{~nm}$ (for the absorbed power $4.7 \mathrm{~mW}$ ). This exclusion holds at a $95 \%$ confidence level.

Thus, in spite of the fact that the dc conductivity of a dielectric-type semiconductor ( $\mathrm{Si}$ in the absence of light) is a real physical phenomenon, its inclusion in the model of dielectric response makes the Lifshitz theory inconsistent with the measurement data. This should be compared with the violation of the Nernst heat theorem in the Lifshitz theory which occurs when the dc conductivity of a dielectric plate is taken into account $\frac{5 \mid 16] 34}{36}$ (see Sec. 1). One can conclude that the inclusion of dc conductivity of dielectric materials makes the Lifshitz theory both theoretically and experimentally inconsistent in analogy with the similar situation for metals discussed in Sec. 1. Phenomenologically, the Lifshitz theory comes to agreement with thermodynamics and is consistent with the experimental data of all experiments performed up to date if one disregards the relaxation processes of conduction electrons in the case of metals $\sqrt{5|16| 85}$ and omits the contribution of charge carriers in the case of dielectrics ${ }^{5|16| 86}$ These prescriptions, however, remain unexplained from basic principles of quantum statistical physics 22

The obtained conclusions were confirmed using another method of comparison between experiment and theory (see Figs. 6 and 8). In Fig. 17(a,b) we plot as dots theoretical minus mean experimental difference Casimir forces, $F_{\text {diff }}^{\text {theor }}(a)-\bar{F}_{\text {diff }}^{\text {expt }}(a)$, for the absorbed powers $9.3 \mathrm{~mW}$ and $4.7 \mathrm{~mW}$, respectively. For the black dots labeled 

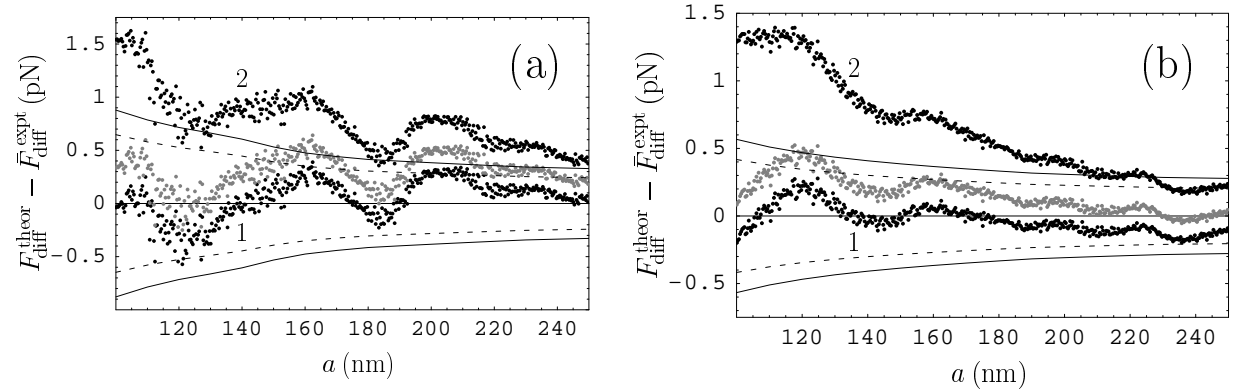

Fig. 17. Theoretical minus mean experimental differences in the Casimir forces are indicated by dots. For black dots labeled 1 and for grey dots dc conductivity of Si in the dark phase is omitted, and charge carriers in the bright phase are described by the generalized plasma-like and Drude-like models, respectively. For black dots labeled 2 dc conductivity of Si in the dark phase is included and charge carriers in the bright phase are described by the generalized Drude-like model. The pairs of the solid and dashed lines indicate the borders of $95 \%$ and $70 \%$ confidence intervals, respectively. The absorbed powers are (a) $9.3 \mathrm{~mW}$ and (b) $4.7 \mathrm{~mW}$.

1, the dc conductivity of a $\mathrm{Si}$ plate in the dark phase is disregarded and charge carriers in the bright phase are described by the generalized plasma-like model (35). In so doing the Au coating on the sphere is also described by means of the generalized plasma-like model (14). For the grey dots, the dc conductivity of Si in the dark phase is also omitted, but charge carriers in the bright phase are described by the generalized Drude-like model (34). To use a uniform approach, Au in this case is described by the tabulated optical data extrapolated by the Drude model. As to the black dots labeled 2, the dc conductivity of a Si plate in the dark phase was taken into account in accordance with Eq. (31). Si in the bright phase was described by the generalized Drude-like model (34) and Au by the tabulated optical data extrapolated by the Drude model.

The pairs of the solid and dashed lines in Fig. 17( $(a, b)$ indicate the borders of the confidence intervals $\left[-\Xi_{0.95}(a), \Xi_{0.95}(a)\right]$ and $\left[-\Xi_{0.7}(a), \Xi_{0.7}(a)\right]$ defined at a $95 \%$ and $70 \%$ confidence levels, respectively. Here, the distribution law for the theoretical minus experimental force differences remains unknown. Because of this, we find the half-width of the confidence interval $\Xi_{0.7}(a)$ from the most conservative assumption of a uniform distribution, i.e., $\Xi_{0.95}(a) / \Xi_{0.7}(a)=0.95 / 0.7 \approx 1.357$ (compare with Sec. 2.1 where the normal distribution was used).

As can be seen in Fig. 17(a,b), all the black dots labeled 1 and most of the grey dots belong to the confidence intervals shown by the solid lines. This means that the two versions of the theory with omitted dc conductivity of Si in the dark phase are experimentally consistent within a 95\% confidence interval. Most of the black dots labeled 1 and the grey dots within some separation intervals [especially in Fig.[17(b)] also belong to the narrower $70 \%$ confidence intervals. This leads to a more definitive conclusion that theoretical approaches with a dc conductivity of $\mathrm{Si}$ in the dark 
phase omitted are experimentally consistent within a $70 \%$ confidence interval. At the same time the experimental data of the optical modulation experiment are not of sufficient precision to convincingly discriminate between the description of free charge carriers in Si in the bright phase by means of the generalized plasma-like and Drude-like models. As to the black dots labeled 2, much more than $95 \%$ of them are outside the borders of the $95 \%$ confidence intervals. Because of this one can conclude that the Lifshitz theory with the inclusion of the dc conductivity of dielectric Si is experimentally excluded at a $95 \%$ confidence level. This is in agreement with similar results obtained above using another method for the comparison between experiment and theory.

Importantly, the same conclusion, that the Lifshitz theory taking into account the dc conductivity of a dielectric material contradicts with the experimental data, was obtained from the measurement of the thermal Casimir-Polder forces between ${ }^{87} \mathrm{Rb}$ atoms and $\mathrm{a} \mathrm{SiO}_{2}$ plate ${ }^{83}$ The theoretical results for the Casimir-Polder force computed 83 with dc conductivity of $\mathrm{SiO}_{2}$ omitted were found 83 in a good agreement with the measurement data. However, the theoretical results computed 87 taking the dc conductivity of $\mathrm{SiO}_{2}$ into account were shown $\frac{87}{}$ to be excluded by the data at a $70 \%$ confidence level. Thus, at the present time there are three types of experiments (with metallic, $17-20$ semiconductor,, $32 \mid 33$ and dielectric $83 \mid 87$ materials) which demonstrate that serious problems arise in the application of the Lifshitz theory in connection with charge carriers.

From a technological point of view, the experiment on optically modulated Casimir force is of special interest. In Sec. 2 we discussed changes of the Casimir force between an Au sphere and an Au plate when the plate material is replaced with different semiconductors. These changes are static and determined by the type of semiconductor used. In the optical modulation experiment the situation is quite different. Here, in the dark phase, the magnitude of the Casimir force between a sphere and a plate is approximately $66 \%$ and $62 \%$ of the Casimir force between an Au sphere and Au plate at $a=100$ and $150 \mathrm{~nm}$, respectively. The increase of the charge carrier density by almost 5 orders of magnitude in the bright phase results in a $3.8 \%$ and $5.6 \%$ increase in the magnitude of the Casimir force at the same respective separations ( $P^{\mathrm{eff}}=9.3 \mathrm{~mW}$ is assumed). The Casimir force is changing periodically until the laser pulses are on. The application of this phenomenon to microdevices moving back and forth without use of mechanical spring under the influence of attractive and repulsive Casimir forces are considered in Sec. 6. The use of the optical modulation of the Casimir force in an ambient environment would be

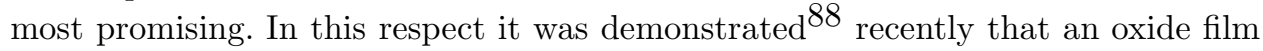
formed on a silicon surface in air hardly affects the possibility of modulating the Casimir force when distances between interacting bodies are of the order of $100 \mathrm{~nm}$. 


\subsection{Comparison with the modified Lifshitz theory}

In the previous section it was demonstrated that the Lifshitz theory including the dc conductivity of dielectric materials is not only thermodynamically inconsistent, but is in conflict with the measurement data of two experiments. To solve this problem, attempts were undertaken $\underline{37-39}$ to modify the Lifshitz theory by including into its formalism screening effects and diffusion currents. According to the proposed modification, 37 the electric field in the dielectric material can be screened due to the presence of free charge carriers with some small density $n$. As a result, the potential around a point charge $e$, instead of a familiar Coulombian form, takes the Yukawa-type form, $e \exp (-\kappa r) / r$, where $\kappa$ is the inverse screening length. If $n$ is sufficiently low, the charge carriers can be described by classical Maxwell-Boltzmann statistics and the Debye-Hückel approximation is valid where

$$
\kappa=\kappa_{\mathrm{DH}}=\frac{1}{R_{\mathrm{DH}}}=\sqrt{\frac{4 \pi e^{2} n}{\varepsilon_{0} k_{B} T}} .
$$

Here, $\varepsilon_{0}=\varepsilon_{\mathrm{ce}}(0)$ is the dielectric permittivity of the core electrons at zero frequency, and $R_{\mathrm{DH}}$ is the screening length. It was assumed that the effect of screening leads to noticeable changes of the reflection coefficients only for the static field. As a consequence, the TM reflection coefficient at zero frequency is replaced with 37

$$
r_{\mathrm{TM}}^{\mathrm{mod}}\left(0, k_{\perp}\right)=\frac{\varepsilon_{0} \sqrt{k_{\perp}^{2}+\kappa^{2}}-k_{\perp}}{\varepsilon_{0} \sqrt{k_{\perp}^{2}+\kappa^{2}}+k_{\perp}} .
$$

At the same time, all of the coefficients $r_{\mathrm{TM}, \mathrm{TE}}\left(i \xi_{l}, k_{\perp}\right)$ with $l \geq 1$ and $r_{\mathrm{TE}}\left(0, k_{\perp}\right)$ defined in Eq. (4) using the dielectric permittivity $\varepsilon_{\mathrm{ce}}\left(i \xi_{l}\right)$ remain unchanged. 37 The modified coefficient (37) should be compared with the standard one, following from Eq. (4)

$$
r_{\mathrm{TM}}\left(0, k_{\perp}\right)=\frac{\varepsilon_{0}-1}{\varepsilon_{0}+1} .
$$

It is evident that Eq. (38) is obtained from Eq. (37) in the limiting case $n \rightarrow 0$.

The modified reflection coefficients for the TM and TE modes at any frequency were obtained 38 through the use of the Boltzmann transport equation, which takes into account not only the drift current $\boldsymbol{j}$, but also the diffusion current $e D \nabla n$, where $D$ is the diffusion constant and $\nabla n$ is the gradient of the charge carrier density. As expected, the TE reflection coefficient at zero frequency remained the same as in Eq. (4) with the generalized Drude-like dielectric permittivity (15). All reflection coefficients $r_{\mathrm{TM}}^{\bmod }\left(i \xi_{l}, k_{\perp}\right)$ with $l \geq 1$ were found approximately equal to $r_{\mathrm{TM}}\left(i \xi_{l}, k_{\perp}\right)$ defined in Eq. (4) to a high degree of accuracy. This approach was also applied 39 to metallic plates, i.e., to bodies with high density of free charge carriers described by the Fermi-Dirac statistics, by replacing the Debye-Hückel screening length with the Thomas-Fermi screening length

$$
\kappa=\kappa_{\mathrm{TF}}=\frac{1}{R_{\mathrm{TF}}}=\sqrt{\frac{6 \pi e^{2} n}{\varepsilon_{0} E_{F}}}
$$


where $E_{F}=\hbar \omega_{p}$ is the Fermi energy.

The proposed modified theory of the van der Waals and Casimir force includes the effect of free charge carriers of the material plates in the reflection coefficients by means of the microscopic quantity $n$ rather than by adding a contribution like in Eq. (31) in the dielectric permittivity. Similar to the standard Lifshitz theory, the modified theory was developed under a condition of thermal equilibrium. However, in contradiction with this condition, the modified theory takes into account the screening effects and both drift and diffusion currents described by means of the Boltzmann transport equation. These physical phenomena are caused by a nonequilibrium distribution of charge carriers in an external field, i.e., by a situation out of thermal equilibrium. It is pertinent to note that the Boltzmann transport equation used to derive the reflection coefficients $r_{\mathrm{TM}, \mathrm{TE}}^{\bmod }(i \xi)$ describes only nonequilibrium processes which must be accompained by an increase of entropy 89

It was shown that the modified theory of the van der Waals and Casimir force violates the Nernst heat theorem for several classes of dielectric materials $5[16] 40 \mid 42[43 \mid 90$ and for metals with perfect crystal lattices.5[16]41|43|91

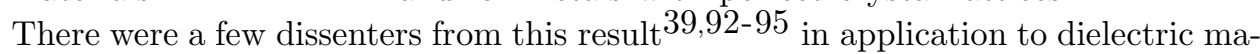
terials. It was noted $\stackrel{22}{22}$ however, that all suggested proofs $39.92-95$ of the validity of the Nernst heat theorem in the modified Lifshitz theory used an assumption that the density of charge carriers in dielectric materials vanishes when the temperature goes to zero. By contrast, the proof of violation of the Nernst theorem in the modified theory was formulated for such dielectric materials as doped semiconductors with doping concentration below critical, dielecric-type semimetals and ionic conductors. For all these materials the density of charge carriers does not vanish when $T \rightarrow 0$ and conductivity vanishes with temperature due to the vanishing mobility. Thus, the modified Lifshitz theory is really in contradiction with thermodynamics for wide classes of materials.

Here we compare theoretical predictions of the modified Lifshitz theory with the experimental data of an experiment on the optically modulated Casimir force. As was noticed in Ref. 40, the comparison of this theory with the experimental data at a $95 \%$ confidence level is not informative because it does not lead to a definite conclusion on the incompatibility with the data. In Fig. 18(a,b) the experimental data for the difference Casimir force for two absorbed powers $(9.3$ and $4.7 \mathrm{~mW}$, respectively) are shown as crosses. The arms of the crosses are twice the respective errors determined at a $70 \%$ confidence level. Keeping in mind that the error $\Delta a$ in the measurement of absolute separation is systematic, the value of this error at a $70 \%$ confidence level was found from the assumption of a uniform distribution: $\Delta a=1 \mathrm{~nm} / 1.375 \approx 0.74 \mathrm{~nm}$ (see Sec. 3.3). The total errors in the force differences are determined by the random errors which are characterized by the normal (or Student with sufficiently large number of degrees of freedom) distribution. Because of this, the absolute total errors in the mean difference Casimir force at a $70 \%$ confidence level were obtained as one half of those shown in Fig.14(a) (see Sec. 3.2). 

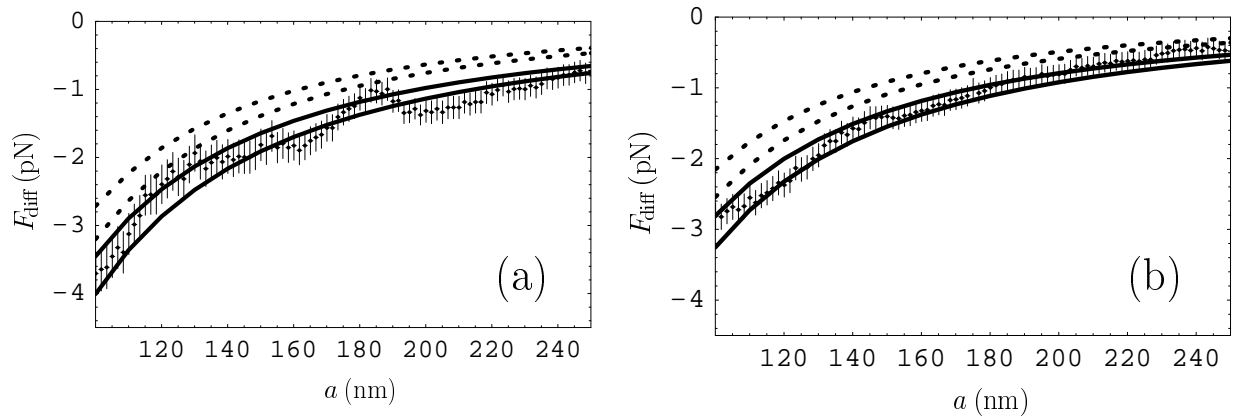

Fig. 18. The experimental differences in the Casimir force are presented as crosses plotted at a $70 \%$ confidence level. The theoretical bands confined between the pairs of solid lines and dashed lines are found at a $70 \%$ confidence level using the standard Lifshitz theory with the dc conductivity of a Si plate in the dark phase neglected and the modified Lifshitz theory, respectively. The absorbed powers are (a) $9.3 \mathrm{~mW}$ and (b) $4.7 \mathrm{~mW}$.

The theoretical bands confined between the pairs of two solid lines were computed using the dielectric permittivity of $\mathrm{Si}, \varepsilon_{\mathrm{ce}}^{(2)}(i \xi)$, in the dark phase and describing Si by the generalized plasma-like model in the bright phase. Au was described by the generalized plasma-like model. The theoretical bands confined between the pairs of two dashed lines were computed using the modified Lifshitz theory ${ }^{38}$ The widths of all theoretical bands were found from the errors in the concentration of charge carriers in the presence of light, $n_{l}$, determined at a $70 \%$ confidence level. Taking into account that the errors in charge carriers are of systematic nature, their values at a $70 \%$ confidence level were obtained using the most conservative assumption of a uniform distribution. The results are $\Delta n_{l}=0.3 \times 10^{19} \mathrm{~cm}^{-3}$ and $\Delta n_{l}=0.22 \times 10^{19} \mathrm{~cm}^{-3}$ for the absorbed powers 9.3 and $4.7 \mathrm{~mW}$, respectively (compare with Sec. 3.1).

As can be seen in Fig. 18(a), the standard Lifshitz theory with the dc conductivity neglected is consistent with the data within a $70 \%$ confidence interval over the range of separations from 100 to $250 \mathrm{~nm}$. The modified Lifshitz theory is excluded by the data at a $70 \%$ confidence level over the same separation region. From Fig. 18(b) one can conclude that the standard Lifshitz theory with neglected dc conductivity is also consistent with the data over all separations, but the modified theory is excluded over the separation region from 100 to $210 \mathrm{~nm}$.

The same results were confirmed using another method of comparison between experiment and theory. In Fig. 19(a,b) the theoretical minus mean experimental difference Casimir forces are indicated as dots. For dots labeled 1, the theoretical differences were computed using the standard Lifshitz theory with neglected dc conductivity of $\mathrm{Si}$ in the dark phase. For $\mathrm{Si}$ in the bright phase and for $\mathrm{Au}$ the generalized plasma-like model has been used. For dots labeled 2, Si in both phases and Au were described by the modified Lifshitz theory 38 The pairs of the solid and dashed lines indicate the borders of $95 \%$ and $70 \%$ confidence intervals, respectively. 

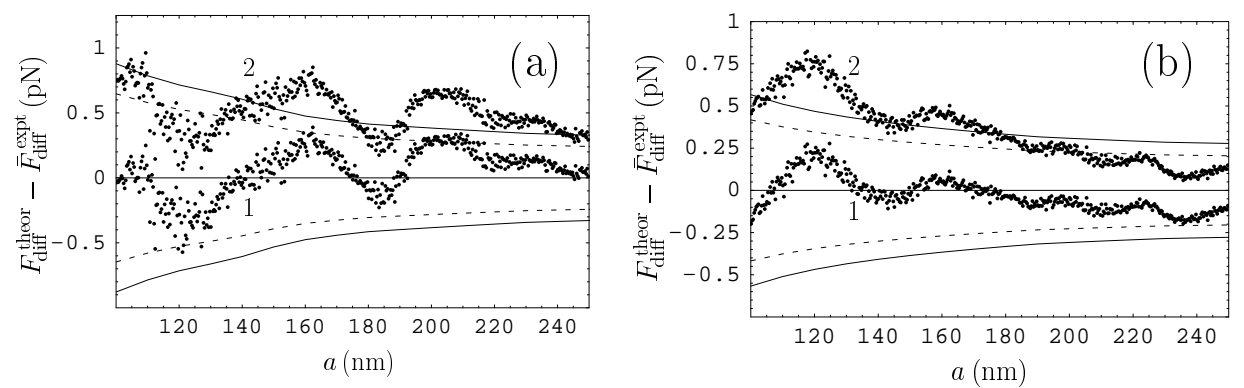

Fig. 19. Theoretical minus mean experimental differences in the Casimir forces are indicated by dots. For dots labeled 1 the standard Lifshitz theory is used with dc conductivity of Si in the dark phase omitted, and charge carriers in the bright phase described by the generalized plasma-like model. For dots labeled 2 theoretical difference Casimir forces are computed using the modified Lifshitz theory. The pairs of the solid and dashed lines indicate the borders of $95 \%$ and $70 \%$ confidence intervals, respectively. The absorbed powers are (a) $9.3 \mathrm{~mW}$ and (b) $4.7 \mathrm{~mW}$.

As is seen in Fig. 19(a,b), the dots labeled 1 belong both to $95 \%$ confidence intervals and [most of dots in Fig.19(a) and all dots in Fig. 19(b)] to 70\% confidence intervals. This means that the standard Lifshitz theory with neglected dc conductivity of a Si plate in the dark phase is experimentally consistent not only within a $95 \%$ confidence interval, but also within a narrower $70 \%$ confidence interval. At the same time, from Fig. 19(a) it is seen that most of dots labeled 2 are outside a $70 \%$ confidence interval (and many of them even outside a $95 \%$ confidence interval) over the entire range of separations from 100 to $250 \mathrm{~nm}$. The same conclusion follows from Fig. 19(b) at separations below $205 \mathrm{~nm}$. This leads to the conservative conclusion that the modified Lifshitz theory is excluded by the measurement data of the experiment on optically modulated Casimir forces at a $70 \%$ confidence level.

It was claimed 39 that the measurement data of the experiment on the optical modulation of the Casimir force are equally consistent with the modified Lifshitz theory and with the standard Lifshitz theory with the dc conductivity neglected in the dark phase. To prove this, the experimental data of Fig. 18(a) at a 70\% confidence level were used, but the theoretical band between the two dashed lines was plotted at a $95 \%$ confidence level using the corresponding uncertainty in the charge carrier density $\Delta n_{l}=0.4 \times 10^{19} \mathrm{~cm}^{-3}$. Such an mismatched comparison was recognized by the author as an error. In the Erratum,, 96 however, both the experimental data and the theoretical bands were plotted at the $95 \%$ confidence level, and the same claim concerning an equal consistency of both theoretical approaches with the data was repeated. This claim disregards the fact that at a $70 \%$ confidence level the theoretical prediction of the modified Lifshitz theory is excluded by the data of experiment on the optical modulation of the Casimir force [see Figs. 18(a,b) and $[19(a, b)]$. As to the comparison with the modified Lifshitz theory at a $95 \%$ confidence level, the data were earlier recognized $\underline{40}$ to lack precision for this com- 
parison to be informative. Note also that in application to metallic test bodies the modified Lifshitz theory was excluded $41-43$ at a $99.9 \%$ confidence level by dynamic measurements of the Casimir pressure using a micromachined oscillator $[19 \mid 20$

\section{Changes of the Casimir force in phase transitions}

An interesting possibility to control the magnitude of the Casimir force due to a change in the charge carrier density is offered by semiconductor materials that undergo a phase transition with change in temperature. This might be used in microdevices as one further possibility to achieve periodic variations of the Casimir force. From a fundamental point of view, the modulation of the Casimir force by phase transitions of various types offers additional test of the role of charge carriers in the Lifshitz theory. It has been known 3 that the variation of the Casimir free energy of a metal associated with the phase transition to the superconducting state is very small. Nevertherless, the magnitude of this variation can be comparable to the condensation energy of a superconducting film and causes a measurable increase in the value of the critical magnetic field $\frac{9798}{16}$ It was proposed 99 also to measure the change of the Casimir force in a superconducting cavity due to a small change in temperature. In semiconductors much greater influence of phase transitions on the Casimir force is expected. Below we consider measurements 44 of Casimir forces between an $\mathrm{Au}$ sphere and AgInSbTe plates in amorphous and crystalline phases. The phase transition occurs with an increase of temperature (annealing). We also discuss the proposal to measure the changes of the Casimir force between an $\mathrm{Au}$ sphere and a vanadium dioxide film which undergoes a dielectric to metal phase transition when the temperature increases.

\subsection{Amorphous and crystalline plates}

This experiment ${ }^{44}$ is of the same type, as discussed in Sec. 2.2, but it was performed in the dynamic regime. The gradient of the Casimir force was measured in succession between an Au-coated sphere of $20.2 \mu \mathrm{m}$ diameter attached to the end of a cantilever of an AFM (see Fig. 1) and two plates consisting of AgInSbTe films deposited onto $\mathrm{Al}$ coated Si wafers. In the first plate a $1 \mu \mathrm{m}$ thick AgInSbTe film was amorphous, and in the second it was annealed to the crystalline state. The cantilever of an AFM was vibrated at its resonant frequency equal to $83.6 \mathrm{kHz}$. When sphere approached to the plate, the frequency shift induced by the Casimir force was measured which is proportional to the gradient of the Casimir force (see Sec. 2.3 for the description of the dynamic regime).

The calibration of the setup was performed by measuring the gradient of the Casimir force with different applied voltages. The residual potential difference $V_{0}$ was found to vary between $0-20 \mathrm{mV}$ for separations $40-150 \mathrm{~nm}$ for both amorphous and crystalline samples. The correction from the residual electrostatic force was subtracted from the data to obtain the measured gradient of the Casimir force for both plates. Typical roughness of the samples was measured using an AFM. It was 
described by a several nanometers rms with a few isolated peaks. The relative experimental error in the force measurements was estimated as about $7 \%$ for both plates at all separations considered. This is in contrast with other dynamic measurements of the Casimir force (see Sec. 2.3 and Refs. 18, 19). The two experimental force curves were obtained (one for the first plate and another for the second) each of which was an average of 13 measurements taken in different areas of both plates. It was concluded that the gradient of the Casimir force for the crystalline plate is greater in magnitude by approximately $20 \%$ than for an amorphous plate within the separation region from 55 to $150 \mathrm{~nm}$.

The experimental results were compared with the theoretically computed using the Lifshitz theory. For this purpose the imaginary parts of the dielectric functions for the crystalline and amorphous films of the AgInSbTe were measured with ellipsometry as a function of frequency in the frequency range from 0.04 to $8.9 \mathrm{eV}$. The $\operatorname{Im} \varepsilon(\omega)$ for a crystalline film exhibiting metallic conductivity was extrapolated to frequencies below $0.04 \mathrm{eV}$ by means of the Drude model. For an amorphous film the contribution from the dc conductivity was neglected. The dielectric permittivities of both films along the imaginary frequency axis were found by means of the Kramers-Kronig relations. The obtained $\varepsilon(i \xi)$ for both films in the frequency region below $20 \mathrm{eV}$ have drastic differences. This was attributed $\frac{44}{4}$ to the structural transformation from the amorphous to the crystalline state alone. It should be noted, however, that the chosen description of dielectric properties disregards free charge carriers that are present in the amorphous state at any nonzero temperature and, thus, interprets this phase transition as occuring from an ideal dielectric to metallic state.

Computations of the gradient of the Casimir forces between an Au-coated sphere and each of the plates were performed using the Lifshitz theory. It is not reported whether the Lifshitz theory at zero temperature or at $T=300 \mathrm{~K}$ (as it was at the laboratory) has been used. This question is in fact of importance for the comparison between the results of computations and the measurement data $\frac{60}{\text { In computations }}$ using the Lifshitz theory the surface roughness was not taken into account. As a justification for the neglect of roughness in computations it was noted that the small roughness of the used samples "is negligible for the Casimir-force computations at separations above $70 \mathrm{~nm}$ [100]". As a result it was found that the Lifshitz theory based on the measured optical properties predicts a force smaller that the measured one by $8-18 \%$. After discussing several reasons which might be responsible for this deviation, which is larger than the experimental and theoretical errors, it was stated somewhat contradictorily that "the observed deviation between theory and experiment can be attributed to surface roughness as discussed recently in Ref. [101."

To conclude, the phase transition from amorphous to crystalline states is a promising subject for further investigations. In principle a semiconductor material can be periodically switched between an amorphous and a crystalline phases by using an appropriate power and duration of the laser pulse ${ }^{44}$ This opens opportunities for measuring the gradient of the difference Casimir force in the configuration 
of an Au-coated sphere above a semiconductor plate undergoing periodic structural changes similar to the experiment on the optically modulated Casimir forces considered in Sec. 3. The combination of a dynamic measurement scheme with the advantages of a difference force measurement can bring much more exact experimental data which should be compared with different theoretical approaches proposed in the literature taking into account nonzero temperature, surface roughness and all other relevant factors.

\subsection{Dielectric to metal transition in $\mathrm{VO}_{2}$}

One more phase transition which leads to a change of the carrier density of order $10^{4}$ is the transition from dielectric to metallic state which occurs with the increase of temperature. Although in the literature it is common to speak about dielectric (insulator) to metal transition, this is in fact a transition between two semiconductor plates with lower $(n)$ and higher $(\tilde{n})$ charge carrier densities. As was shown above, an increase of $n$ by a factor of $10^{4}$ leads to measurable changes in the Casimir force. Thus, the phase transition from dielectric to metal phase can be used to control the Casimir force in a sphere-plate geometry by mere temperature change.

It was proposed $\frac{45}{40}$ measure the change of the Casimir force acting between an $\mathrm{Au}$-coated sphere and a vanadium dioxide $\left(\mathrm{VO}_{2}\right)$ film deposited on a sapphire substrate. $\mathrm{VO}_{2}$ films are monoclinic at room temperature and can be considered as semiconductors of the dielectric type as their conductivity vanishes with vanishing temperature. At $68^{\circ} \mathrm{C}$ the resistivity of $\mathrm{VO}_{2}$ films abruptly decreases by a factor of $10^{4}$ from 10 to $10^{-3} \Omega \mathrm{cm}$. This is a consequence of the phase transition to a metallic tetragonal phase ${ }^{102}$ In the process, the optical transmission of $\mathrm{VO}_{2}$ for wavelength from $1 \mu \mathrm{m}$ to greater than $10 \mu \mathrm{m}$ decreases by a factor $10-100$. Thus, in this case we deal with the structural transformations between the two different crystalline phases, whereas in Sec. 4.1 the transformation from an amorphous to a crystalline phase was considered.

The schematic of the experimental setup suitable for the observation of the difference Casimir force in the dielectric to metal phase transition is similar to that shown in Fig. 11. Main differences are that the specially fabricated Si plate should be replaced with $\mathrm{VO}_{2}$ film on a sapphire substrate and, instead of a $514 \mathrm{~nm}$ Ar laser, a chopped $980 \mathrm{~nm}$ laser should be used to heat the $\mathrm{VO}_{2}$ film.103|104 Estimations show that approximately $10-100 \mathrm{~mW}$ of power of the $980 \mathrm{~nm}$ laser are required to bring about all the optical switching of a $\mathrm{VO}_{2}$ film. Here, as opposed to Sec. 3, the wavelength of the laser was chosen in such a way that light only leads to heating of the $\mathrm{VO}_{2}$ film, but does not change the number of free charge carriers.

The proposed experiment on the measurement of the difference Casimir force in the dielectric to metal phase transition is of much interest for actuation of nanodevices through a modulation of the Casimir force, and for further tests of the Lifshitz theory. The latter demands precision measurements of the Casimir force in the configuration of an $\mathrm{Au}$-coated sphere and $\mathrm{VO}_{2}$ film and careful comparison 
between the experimental data and theory.

The Lifshitz theory expresses the Casimir force both before (the density of charge carriers $n$ ) and after (the density of charge carriers $\tilde{n}$ ) the phase transition by Eqs. (3) and (10). To compute the Casimir force both before and after the phase transition one needs the optical data of $\mathrm{VO}_{2}$ on a sapphire plate in a wide frequency region. Such data were measured 105 and used in the fit to the oscillator model of the dielectric permittivity in the frequency region from 0.25 to $5 \mathrm{eV}$. The additional terms were also added 45 to the dielectric permittivity taking into account the contributions from the optical data at higher frequencies up to about $10 \mathrm{eV}$. As a result, the effective dielectric permittivity of the $\mathrm{VO}_{2}$ film on a sapphire substrate at the imaginary Matsubara frequencies before the phase transition (at $T=300 \mathrm{~K}$ ) takes the form

$$
\varepsilon_{n}\left(i \xi_{l}\right)=1+\sum_{i=1}^{7} \frac{s_{n, i} \omega_{n, i}^{2}}{\omega_{n, i}^{2}+\xi_{l}^{2}+\Gamma_{n, i} \xi_{l}}+\frac{\left(\varepsilon_{\infty}^{(n)}-1\right) \omega_{\infty}^{2}}{\omega_{\infty}^{2}+\xi_{l}^{2}} .
$$

Here, $\varepsilon_{\infty}^{(n)}=4.26, \omega_{\infty}=15 \mathrm{eV}$ and the values of all oscillator parameters can be found in Refs. 45] and 105. In Fig. 20(a) the dielectric permittivity (40) as a function of frequency is shown by the solid line labeled 1. In the same figure, the dielectric permittivity of $\mathrm{Au}$ computed using the generalized plasma-like model is shown as dots. The vertical line indicates the position of the first Matsubara frequency at $T=340 \mathrm{~K}$, i.e., in the region of the phase transition.

After the phase transition the $\mathrm{VO}_{2}$ film is in metallic state with increased charge carrier density $\tilde{n}$. In this case the effective dielectric permittivity of the $\mathrm{VO}_{2}$ film on a sapphire substrate is given by

$$
\varepsilon_{\tilde{n}}\left(i \xi_{l}\right)=1+\frac{\omega_{p, \tilde{n}}^{2}}{\xi_{l}\left(\xi_{l}+\gamma_{\tilde{n}}\right)}+\sum_{i=1}^{7} \frac{s_{\tilde{n}, i} \omega_{n, i}^{2}}{\omega_{\tilde{n}, i}^{2}+\xi_{l}^{2}+\Gamma_{\tilde{n}, i} \xi_{l}}+\frac{\left(\varepsilon_{\infty}^{(\tilde{n})}-1\right) \omega_{\infty}^{2}}{\omega_{\infty}^{2}+\xi_{l}^{2}}
$$

The values of the oscillator parameters used here can be found in Refs. 45 and 105 , whereas the other parameters are ${ }^{105} \varepsilon_{\infty}^{(\tilde{n})}=3.95, \omega_{p, \tilde{n}}=3.33 \mathrm{eV}$, and $\gamma_{\tilde{n}}=0.66 \mathrm{eV}$. In Fig. 20(a) the dielectric permittivity (41) is shown by the solid line labeled 2 .

The computational results for the Casimir force between an Au-coated sphere and $\mathrm{VO}_{2}$ film deposited on a sapphire plate versus separation were obtained $\sqrt{45}$ using the dielectric permittivity (40) before the phase transition and dielectric permittivity (41) after the phase transition. They are shown in Fig. 20(b) by the solid lines 1 and 2, respectively. As can be seen in Fig. 20(b), after the phase transition the magnitude of the Casimir force increases due to an increase in the charge carrier density and structural changes of the material. Specifically, the difference Casimir force from a phase transition changes from $13 \mathrm{pN}$ at $a=100 \mathrm{~nm}$ to $1.2 \mathrm{pN}$ at $a=300 \mathrm{~nm}$. Note that the Casimir forces between an $\mathrm{Au}$-coated sphere and $\mathrm{VO}_{2}$ film deposited on a sapphire were also computed $\frac{106}{}$ not using the effective dielectric permittivities, as above, but considering instead a two layer system of $\mathrm{VO}_{2}$ and sapphire with their individual permittivities. However, computations of Casimir forces in Ref. 106 were performed in the framework of the Lifshitz theory at zero temperature. 

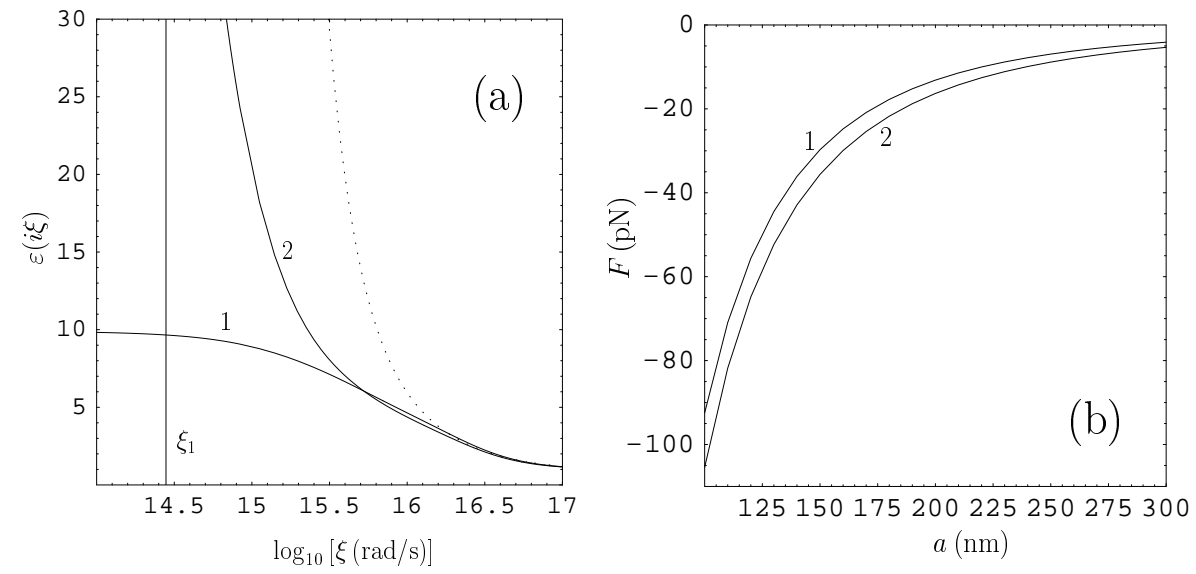

Fig. 20. (a) The effective dielectric permittivity of $\mathrm{VO}_{2}$ film on sapphire substrate along the imaginary frequency axis before and after the phase transition are shown by the solid lines 1 and 2, respectively. The permittivity of $\mathrm{Au}$ is indicated by the dotted line. (b) The Casimir force between an $\mathrm{Au}$ coated sphere and $\mathrm{VO}_{2}$ film on sapphire substrate versus separation before and after the phase transition are shown by the solid lines 1 and 2, respectively.

The difference Casimir force in the dielectric-to-metal phase transition can be used as one more test of the Lifshitz theory. Similar to Sec. 3, we arrive at different results for the difference Casimir force depending on whether or not the dc conductivity of the dielectric $\mathrm{VO}_{2}$ before the phase transition was taken into account in computations. The quantity equal to the difference Casimir force with neglected dc conductivity of dielectric $\mathrm{VO}_{2}$ minus the difference Casimir force with dc conductivity included varies from $1.6 \mathrm{pN}$ at $a=100 \mathrm{~nm}$ to $0.2 \mathrm{pN}$ at $a=300 \mathrm{~nm}$. This can be used to experimentally discriminate between the two approaches and deeply probe the role of material properties in the Lifshitz theory at nonzero temperature.

\section{Semiconductor plate with rectangular corrugations}

In this section we discuss two experiments 46477 on the measurement of the gradient of the Casimir force between an Au-coated sphere of a radius $R=50 \mu \mathrm{m}$ and a Si surface that has been structured with nanoscale rectangular corrugations (trenches). Measurements were performed in the dynamic regime using a micromechanical torsional oscillator. This means that the directly measured quantity was the shift of the resonant frequency of the oscillator, which is proportional to the gradient of the Casimir force. Using Eq. (17) this gradient can be recalculated into the Casimir pressure between the two plates one of which is flat and another one rectangular corrugated. In both experiments the trenches were fabricated in $p$-type silicon with the density of charge carriers $2 \times 10^{18} \mathrm{~cm}^{-3}$ and respective resistivity equal to $0.028 \Omega \mathrm{cm}$. Note that this density of charge carriers is a bit smaller than the critical value above which Si becomes a metallic-type semiconductor. 


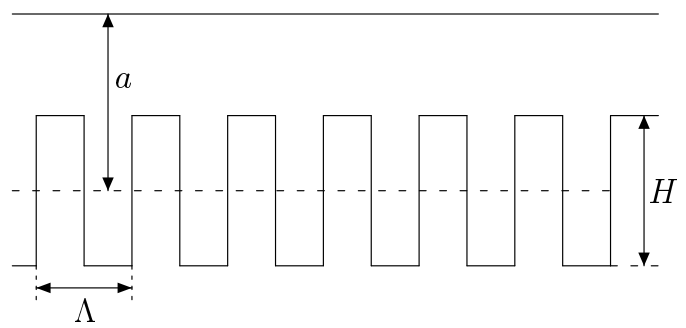

Fig. 21. The periodic uniaxial rectangular corrugations on one of the plates.

The configuration of two plates with periodic rectangular corrugations on one of them used in the first experiment $\frac{46}{16}$ is shown in Fig. 21] The depth of the trenches was $H \approx 1 \mu \mathrm{m}$. Three types of lower plate of size $0.7 \times 0.7 \mathrm{~mm}^{2}$ were made: sample A with a period $\Lambda_{\mathrm{A}}=1 \mu \mathrm{m}$, sample B with a period $\Lambda_{\mathrm{B}}=400 \mathrm{~nm}$ and one additional sample with a flat surface. The sidewalls of the trenches A and B were described as having angles of $90.3^{\circ}$ and $91.0^{\circ}$, respectively, with the top surfaces. The bottom corners were reported to have some degree of rounding in comparison with the top corners. Perfect corners of $90^{\circ}$ were assumed in all of the analyses. Fractional areas of the top surfaces of the unetched regions were found to be $p_{\mathrm{A}}=0.478 \pm 0.002$ and $p_{\mathrm{B}}=0.510 \pm 0.001$ for the samples A and B using a scanning electron microscope.

The electrostatic calibrations and the measurements of the gradient of the Casimir force were done in a vacuum of $10^{-6}$ Torr. A residual potential difference $V_{0} \sim-0.43 \mathrm{~V}$ between the sphere and the flat Si plate was noted to vary by $3 \mathrm{mV}$ over the range of separations from $600 \mathrm{~nm}$ to $2.5 \mu \mathrm{m}$. Since no analytic expression for the electrostatic force is available for a trench geometry, a $2 D$ numerical solution of the Poisson equation was used to calculate the electrostatic energy between a flat plate and a trench surface. This energy was then converted to a force between a sphere and the trench surface using the PFA.

The Casimir force gradients between the sphere and the flat plate and samples A and $\mathrm{B}$ were measured at the laboratory temperature $T=300 \mathrm{~K}$ after the application of compensating voltages to the plates. The main uncertainty in these measurements

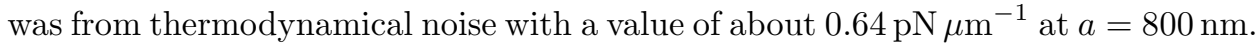
The gradient of the Casimir force between the flat plate and an Au sphere $F_{\text {flat }}^{\prime}$ was measured first. Good agreement was claimed between these measurement results and the Lifshitz theory at zero temperature using the tabulated optical data ${ }^{51}$ for $\mathrm{Au}$ and Si extrapolated to low frequencies by means of the Drude model. There are, however, some deviations between the data and the computed force gradient (note that large effect from the inclusion of dc conductivity is more pronounced when the Lifshitz formula at nonzero temperature is used in computations). The roughness correction was taken into account employing an rms roughness of $4 \mathrm{~nm}$ on the sphere and $0.6 \mathrm{~nm}$ on the silicon surface measured by means of an AFM. Next, the force 
gradients $F_{\mathrm{A} \text {,expt }}^{\prime}$ and $F_{\mathrm{B} \text {,expt }}^{\prime}$ were measured on the corrugated surfaces.

The measurement results were compared with theory at zero temperature. For the configuration with deep rectangular corrugations one can neglect the contribution of the remote bottom parts of the trenches. Then the PFA leads to the following results for the samples $\mathrm{A}$ and $\mathrm{B}$

$$
F_{\mathrm{A}, \mathrm{PFA}}^{\prime}(a)=-2 \pi R p_{\mathrm{A}} P_{r}\left(a-\frac{H}{2}\right), \quad F_{\mathrm{B}, \mathrm{PFA}}^{\prime}(a)=-2 \pi R p_{\mathrm{B}} P_{r}\left(a-\frac{H}{2}\right),
$$

where $P_{r}$ is the Casimir pressure between two parallel noncorrugated plates calculated by the Lifshitz formula (9) taking into account stochastic roughness. To compare the experimental data with theory the following ratios were considered $\frac{46}{46}$

$$
\rho_{\mathrm{A}}(a)=\frac{F_{\mathrm{A}, \operatorname{expt}}^{\prime}(a)}{F_{\mathrm{A}, \mathrm{PFA}}^{\prime}(a)}, \quad \rho_{\mathrm{B}}(a)=\frac{F_{\mathrm{B}, \mathrm{expt}}^{\prime}(a)}{F_{\mathrm{B}, \mathrm{PFA}}^{\prime}(a)} .
$$

It was found that for sample A there were deviations of $\rho_{\mathrm{A}}$ from unity of up to $10 \%$ over the measurement range from $a=650$ to $750 \mathrm{~nm}$, exceeding the experimental errors. For sample B, there were deviations of $\rho_{\mathrm{B}}$ from unity up to $20 \%$ over the same measurement range. These deviations are natural as $a / \Lambda_{\mathrm{A}}=0.7$ and $a / \Lambda_{\mathrm{B}}=1.75$ at the typical separation considered, $a=700 \mathrm{~nm}$. As discussed in the literature, $\underline{5[16] 107}$ the application region of the PFA to periodic structures is restricted by an additional condition $a / \Lambda \ll 1$, where $\Lambda$ is the period. Thus, for sample B the applicability condition of the PFA is violated to a larger extent than for sample A.

The experimental data were also compared with exact theoretical values 108 computed for ideal metal corrugations at zero temperature. In so doing the PFA was not applied to corrugations, but used only to convert the plate-plate to sphereplate case. However, the above measured deviations from the PFA were found to be $50 \%$ less than calculated for ideal metal boundaries. This discrepancy was reported as being quite natural, owing to the fact that exact calculation did not take into account nonzero skin depth of both $\mathrm{Au}$ and Si surfaces. Furthermore, exact calculations for a corrugated plate were performed 109 at zero temperature where $\mathrm{Au}$ was described by the simple plasma model and the Si grating by the generalized Drude-like model 106 This allowed to decrease discrepancies between experiment and theory.

The second experiment 47 on the measurement of the Casimir force gradient between an Au sphere and a plate covered with rectangular corrugations was performed with smaller corrugation depth. In this case, unlike the first experiment, both top and bottom surfaces of the corrugations contributed to the force gradient. For the second esperiment, the same $p$-type $\mathrm{Si}$ was used as in the first with the parameters indicated above. The period of corrugations was $\Lambda=400 \mathrm{~nm}$. The sidewalls of corrugations were at $94.6^{\circ}$ to the top surface, i.e., not exactly vertical. As a result, the lengths of the top surface and the bottom surface in one period were measured to be $l_{1}=185.3 \mathrm{~nm}$ and $l_{2}=199.1 \mathrm{~nm}$, respectively. The average depth of corrugations was $H=97.8 \pm 0.7 \mathrm{~nm}$ (i.e., an order of magnitude less than in the 
first experiment). Another sample consisted of a flat surface with no corrugations was also used with the same optical properties.

The same micromechanical torsional oscillator was used in the dynamic regime to measure the gradient of the Casimir force. The sphere radius was $R=50 \mu \mathrm{m}$. Voltages were applied to the flat plate for calibration purposes while the sphere remained grounded. The residual voltage was found to be $V_{0}=-0.499 \mathrm{~V}$ from the fit to the exact force-distance relation. It was found to change only by less than $3 \mathrm{mV}$ for $a$ changing from 150 to $650 \mathrm{~nm}$. Similar calibration procedure was repeated for a corrugated Si plate, but instead of an analytic force-distance relation the fit to a numerical solution of the Poisson equation was performed. Next, the gradients of the Casimir force, $F_{f, \text { expt }}^{\prime}(a)$ and $F_{c \text {,expt }}^{\prime}(a)$, on the flat and corrugated surfaces, respectively, were measured by applying the compensating voltages to the plate in order to compensate the residual potential difference $V_{0}$.

The obtained experimental data were compared with the results of theoretical computations. For a flat plate $F_{f, \text { theor }}^{\prime}(a)$ was computed using Eq. (17) and the Lifshitz formula for the Casimir pressure at zero temperature. In so doing $\mathrm{Si}$ was described by the generalized Drude-like model (15) with the Drude parameters $\omega_{p}^{(2)}=1.36 \times 10^{14} \mathrm{rad} / \mathrm{s}$ and $\gamma^{(2)}=4.75 \times 10^{13} \mathrm{rad} / \mathrm{s}$. Au was described by the tabulated optical data extrapolated to low frequencies by means of the Drude model with the parameters indicated in Sec. 2.1. The surface roughness $(\sim 4 \mathrm{~nm}$ rms for an $\mathrm{Au}$ surface and $\sim 0.6 \mathrm{~nm}$ rms for a Si surface) was taken into account by the method of geometrical averaging. $\frac{5[16] 18 / 27}{5}$ The comparison between the data, $F_{f \text {,expt }}^{\prime}(a)$, and the computational results, $F_{f, \text { theor }}^{\prime}(a)$, corrected to a presence of surface roughness, shows that there is some disagreement (the measured force gradients are less than the computed ones). This can be explained by the use of the Lifshitz formula at zero temperature in computations, whereas the experiment was performed at $T=300 \mathrm{~K}$. The related differences are of about $-0.5 \%$ or $1 \%$ depending on the model of conductivity properties of $\mathrm{Si}$ used at nonzero temperature.

Now we discuss the comparison between experiment and theory for a corrugated plate. Here, the approximate expression for the gradient of the Casimir force can be simply obtained using the PFA from Eq. (1) of Ref. 47. The result is:

$$
\begin{array}{rl}
F_{c, r ; \mathrm{PFA}}^{\prime}(a)=-2 & R\left\{p_{1} P_{r}\left(a-H_{1}\right)+p_{2} P_{r}\left(a+H_{2}\right)\right. \\
+ & \left.\frac{1-p_{1}-p_{2}}{H}\left[E_{r}\left(a-H_{1}\right)-E_{r}\left(a+H_{2}\right)\right]\right\} .
\end{array}
$$

Here, similar to Eq. (42), $P_{r}$ is the Casimir pressure between two parallel noncorrugated plates at $T=0$ calculated by the Lifshitz formula (9) with account of surface roughness. The Casimir energy $E_{r}$ is given by the Lifshitz formula (8) and is also corrected for the presence of roughness using the method of geometrical averaging. The quantities $p_{1}$ and $p_{2}$ are defined as $p_{1}=l_{1} / \Lambda, p_{2}=l_{2} / \Lambda$, and $H_{i}=\left(\Lambda-l_{i}\right) /\left(2 \Lambda-l_{1}-l_{2}\right)$. The first and second contributions on the right-hand side of Eq. (44) describe the role of the top and bottom surfaces of the Si grating, 
respectively. They contribute about $97 \%$ of the Casimir force computed using the PFA.

The comparison between the data, $F_{c, \text { expt }}^{\prime}(a)$, and the computational results, $F_{c, \text { theor }}^{\prime}(a)$, corrected to a presence of surface roughness, shows that the measured force gradients are a bit larger than the computed ones. This again might be partially connected with the use of the Lifshitz formula at zero temperature. Note that the PFA result for the gradient of the Casimir force at any $T \neq 0$ is obtained from Eq. (44) by using the pressures $P_{r}$ calculated using the Lifshitz formula at nonzero temperature and by replacing the energy $E_{r}$ with the free energy $\mathcal{F}_{r}$. With the increase of separation from $a=0.2$ to $0.55 \mu \mathrm{m}$ the experimental data were found to be in better agreement with the PFA results. Specifically, the ratio

$$
\rho(a)=\frac{F_{c, \operatorname{expt}}^{\prime}(a)}{F_{c, r ; \mathrm{PFA}}^{\prime}(a)}
$$

was equal 47 to approximately 1.15 at $a=200 \mathrm{~nm}$ and 1 at $a=0.53 \mu \mathrm{m}$ (our definition of separation $a$ counted from the mean level of corrugations is connected with that used in Ref. 47 as $a \approx z+50 \mathrm{~nm}$ ).

The gradient of the Casimir force between an Au sphere and corrugated Si plate, $F_{c, \text { theor }}^{\prime}(a)$, was also computed using the exact scattering approach at $T=0$. It was shown that the ratio

$$
\rho^{\text {theor }}(a)=\frac{F_{c, \text { theor }}^{\prime}(a)}{F_{c, r ; \text { PFA }}^{\prime}(a)}
$$

varies from 1.1 at $a=200 \mathrm{~nm}$ to 1.05 at $a=550 \mathrm{~nm}$. This was characterized as a good agreement with variations of the quantity $\rho(a)$ indicated above. It should be noted, however, that both quantities, $F_{c, r ; \mathrm{PFA}}^{\prime}(a)$ in the denominator of $\rho(a)$ and $\rho^{\text {theor }}(a)$, were computed at $T=0$ while the measurements were performed at $T=300 \mathrm{~K}$. For real materials the quantity $\rho^{\text {theor }}(a)$ decreases towards unity with the increase of $a$ just as for the case of ideal metals. ${ }^{108}$ Note that at separations $a<$ $270 \mathrm{~nm}$ there is some disagreement between the exact theory and the experimental data [because the differences $\rho(a)-\rho^{\text {theor }}(a)$ exceed the experimental errors]. This might be explained by the slow convergence of computations in the exact theory and insufficient number of iterations used.

All in all, the second experiment using an Au sphere interacting with a Si corrugated plate provided further evidence concerning an interplay between geometry effects and material properties in the case of rectangular corrugated surfaces. The Casimir force measured at $T=300 \mathrm{~K}$ was found to deviate from the PFA computations at $T=0 \mathrm{~K}$ by about $10 \%$. It is pertinent to note that the quantitative comparison at a $95 \%$ confidence level between the measurement data, the exact computational results using the scattering approach, and the predictions of the

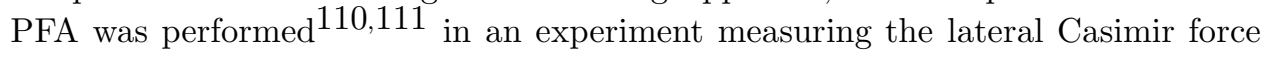
in the configuration of a sinusoidally corrugated sphere and a sinusoidally corrugated plate. In this experiment both test bodies were metallic (coated with $\mathrm{Au}$ ). 
The experimental data were found to be in excellent agreement with the results of exact computations using the scattering approach, but deviate significantly from the results of the PFA computations. In so doing the exact computations were performed at the laboratory temperature $T=300 \mathrm{~K}$. This provided the quantitative confirmation of the observation of diffraction-type effects in the Casimir force that are disregarded within the PFA approach. Note that for a lateral Casimir force the quantity analogous to $\rho^{\text {theor }}$ in Eq. (46),

$$
\rho_{\text {lat }}^{\text {theor }}(a)=\frac{F_{c, t \text { theor }}^{\text {lat }}(a)}{F_{c, r ; \mathrm{PFA}}^{\text {lat }}(a)},
$$

is less than unity. According to the computational results, $110\left[111 \rho_{\text {lat }}^{\text {theor }}(a)\right.$ decreases with the increase of $a$, i.e., for the lateral Casimir force the accuracy of the PFA computations decreases with the increase of separation. This is because the lateral force is determined by the dependence of the Casimir energy on the phase shift between the corrugations.

\section{Semiconductors in three-layer systems: Pulsating Casimir force}

In all experiments using semiconductor test bodies discussed above, the Casimir force was always attractive. The possibility to realize a repulsive Casimir force attracts much attention due to potential applications in nanotechnology to avoid stiction of closely positioned microelements and to actuate the periodic movement in electro- and optomechanical micromachines. Up to the present, however, most of the proposals on this subject were far from experimental realization. The repulsive Casimir forces have long been discussed for an ideal metal sphere ${ }^{5}$ and ideal metal rectangular boxes with appropriate ratio of side lengths. $[5 \mid 112$ These results, however, were not generalized for spheres and boxes made of real metals. Numerous speculations on the possibility of Casimir repulsion between artificial metamaterials have recently been proven to be not realizable.113[114

The single realistic possibility for the observation of the Casimir repulsion in three-layer systems was proposed long ago. It was noticed 7 that repulsion happens when the two semispaces (plates) with the dielectric permittivities along the imaginary frequency axis $\varepsilon^{(2)}(i \xi)<\varepsilon^{(1)}(i \xi)$ are separated with a material layer having the dielectric permittivity $\varepsilon^{(0)}(i \xi)$ such that

$$
\varepsilon^{(2)}(i \xi)<\varepsilon^{(0)}(i \xi)<\varepsilon^{(1)}(i \xi) .
$$

To obtain repulsion, inequality (48) should hold over a sufficiently wide frequency region. This can be achieved by using a liquid intermediate layer with the dielectric permittivity $\varepsilon^{(0)}(i \xi)$. Experimentally the effect of Casimir repulsion was demonstrated 115,118 in sphere-plate geometry using $\alpha$-alumina and Au spheres, amorphous $\mathrm{SiO}_{2}$ plates and different liquids (cyclohexane, bromobenzene etc.) at separations up to about $10 \mathrm{~nm}$. Note that at such separations the relativistic retardation effects are already important. The Casimir repulsion between an Au sphere 
and a $\mathrm{SiO}_{2}$ plate with an intermediate layer of bromobenzene was observed 119 at separations of about $30 \mathrm{~nm}$. Some problems which add complexity to measurements of the Casimir force in liquids, specifically, the formation of a double layer owing to salt impurities, are discussed in Ref. 120. Here, we consider how semiconductor can be employed in three-layer systems for the realization of a pulsating Casimir

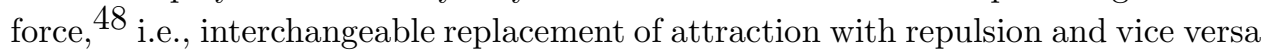
without use of mechanical springs.

Let us consider three pairs of plates immersed in ethanol. The first pair includes one $\mathrm{Au}$ and one $\mathrm{Si}$ plate and the Si plate is illuminated by laser pulses as in the experiment on the optically modulated Casimir force discussed in Sec. 3. The second pair consists of two similar Si plates, and only one of them is illuminated with laser pulses. According to Sec. 3, the illumination of Si with light of appropriate power and wavelength increases the charge carrier density by four orders of magnitude and changes the dielectric permittivity along the imaginary frequency axis over a wide frequency range. This can be used to satisfy Eq. (48) or, on the contrary, to violate it when it was satisfied in the absence of light. In the third pair of plates immersed in ethanol, we consider one plate made of $\alpha-\mathrm{Al}_{2} \mathrm{O}_{3}$ and the other of Si. The latter plate is also illuminated with laser pulses. Computations of the Casimir force show that for the first pair of plates there is a repulsive Casimir force within a wide range of separations when the light is off and an attractive force when the light is on. For the second and third pair of plates, the force is repulsive when the light is on and attractive when the light is off.

To perform these computations the Lifshitz formula for the Casimir pressure in

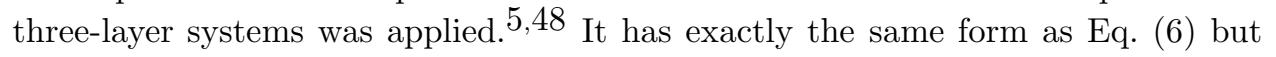
the reflection coefficients (4) should be replaced with

$$
\begin{aligned}
r_{\mathrm{TM}}^{(n)} & =\frac{\varepsilon^{(n)}\left(i \xi_{l}\right) k_{l}^{(0)}-\varepsilon^{(0)}\left(i \xi_{l}\right) k_{l}^{(n)}}{\varepsilon^{(n)}\left(i \xi_{l}\right) k_{l}^{(0)}+\varepsilon^{(0)}\left(i \xi_{l}\right) k_{l}^{(n)}}, \\
r_{\mathrm{TE}}^{(n)} & =\frac{k_{l}^{(0)}-k_{l}^{(n)}}{k_{l}^{(0)}+k_{l}^{(n)}}, \quad k_{l}^{(0)} \equiv\left[k_{\perp}^{2}+\varepsilon^{(0)}\left(i \xi_{l}\right) \frac{\xi_{l}^{2}}{c^{2}}\right]^{1 / 2} .
\end{aligned}
$$

To calculate the Casimir pressure for the above three pairs of plates, in addition to the dielectric permittivities of $\mathrm{Au}$ and $\mathrm{Si}$ discussed in Secs. 2 and 3, one needs the dielectric permittivities of $\alpha-\mathrm{Al}_{2} \mathrm{O}_{3}$ and ethanol along the imaginary frequency axis. They can be presented in the Ninham-Parsegian form $121 \mid 122$

$$
\varepsilon^{(n)}\left(i \xi_{l}\right)=1+\frac{C_{n}^{\mathrm{IR}}}{1+\xi_{l}^{2} / \omega_{\mathrm{IR}, n}^{2}}+\frac{C_{n}^{\mathrm{UV}}}{1+\xi_{l}^{2} / \omega_{\mathrm{UV}, n}^{2}},
$$

where the values of all parameters for $\alpha-\mathrm{Al}_{2} \mathrm{O}_{3}(n=1)$ and for ethanol $(n=0)$ can be found in Refs. 48, 121, 122, In Fig. 22 we show all related dielectric permittivities along the imaginary frequency axis. The dielectric permittivities of $\mathrm{Si}$ in the absence and in the presence of light are indicated by the solid lines 1 and 2, respectively. The line 2 is plotted for the absorbed power $P^{\text {eff }}=9.3 \mathrm{~mW}$ (see Sec. 3 ). The dielectric 


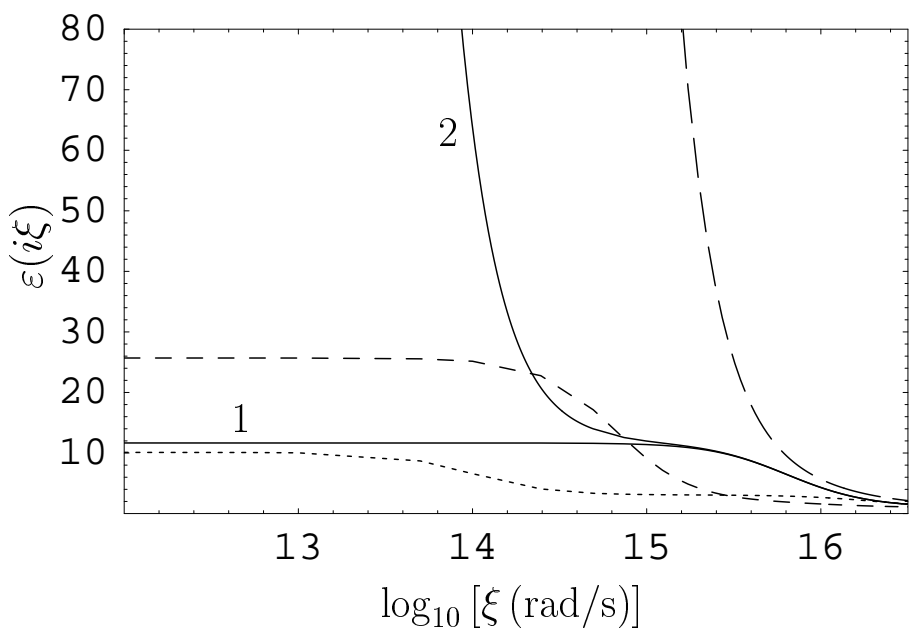

Fig. 22. The dielectric permittivities of different materials along the imaginary frequency axis are shown with solid lines 1 and 2 for $\mathrm{Si}$ in the absence and in the presence of laser light, respectively, with a long-dashed line for $\mathrm{Au}$, with a short-dashed line for ethanol, and with a dotted line for $\alpha-\mathrm{Al}_{2} \mathrm{O}_{3}$.

permittivities of $\mathrm{Au}$, ethanol, and $\alpha-\mathrm{Al}_{2} \mathrm{O}_{3}$ are presented by the long-dashed, shortdashed, and dotted respective lines.

In Fig. 23(a) we present the computational results for the Casimir pressure versus separation for the first (the solid lines) and second (the dashed lines) pairs of plates. For the first pair of plates, i.e., for Au and Si plates separated by ethanol, the pressure-distance dependence in the absence of light on a Si plate is shown as the black solid line. As can be seen in Fig. 23(a), for separations larger than $156 \mathrm{~nm}$
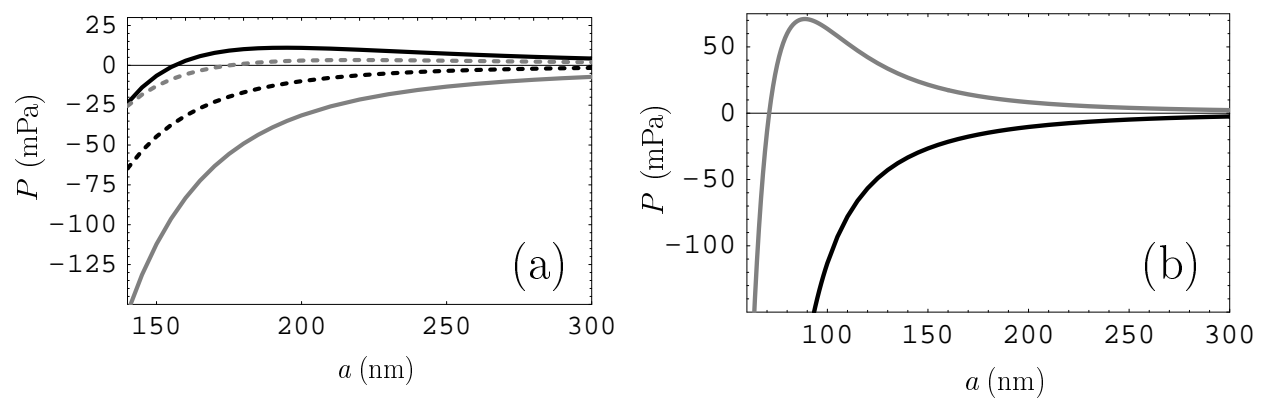

Fig. 23. The Casimir pressure versus separation in three-layer systems (a) Au-ethanol-Si (the black and grey solid lines are for the absence and presence of light on the Si plate, respectively) and $\mathrm{Si}$-ethanol-Si (the black and grey dashed lines are for the absence of light on both Si plates and for the presence of the light on one of them); (b) $\alpha-\mathrm{Al}_{2} \mathrm{O}_{3}$-ethanol-Si (the black and grey lines are for the absence and presence of light on the Si plate, respectively). 
the Casimir pressure shown by the black solid line is repulsive. This reflects the fact that in Fig. 22 the dielectric permittivity of ethanol is sandwiched between the dielectric permittivities of $\mathrm{Si}$ and $\mathrm{Au}$ in a wide frequency range, i.e., inequalities (48) are satisfied. The pressure-distance dependence in the presence of laser light on a Si plate is shown by the grey solid line in Fig. 23(a). This line corresponds to attraction at all separation distances. Physical explanation of this fact is seen in Fig. 22 where the dielectric permittivity of Si in the presence of light (the solid line 2) is larger than the dielectric permittivity of ethanol (the short-dashed line) within a wide frequency region. Thus, the illumination of a Si plate with laser light violates inequalities (48) and changes repulsion for attraction.

For the second pair of plates, i.e., for the two Si plates separated by ethanol, the Casimir force in the absence of laser light is attractive at all separations [see the dashed black line in Fig. 23(a)]. This result is expected because the permittivities of both plates are equal. When, however, one Si plate is illuminated with a laser light of appropriate wavelength and power, its dielectric permittivity is described by the solid line labeled 2 in in Fig. 22, and the inequalities (48) are satisfied. In this case, the computational results for the Casimir pressure versus separation are shown by the dashed grey line in Fig. 23(a). As can be seen in this figure, at $a<175 \mathrm{~nm}$ the Casimir force is attractive, but at $a>175 \mathrm{~nm}$ it is repulsive. Thus, the illumination of one of the two Si plates separated by ethanole with light changes the Casimir attraction to Casimir repulsion.

For the first and second pairs of plates, the magnitudes of the repulsive forces were several times less than the magnitudes of the attractive forces. This is not the case for the third pair of plates, i.e., for the three-layer system $\alpha-\mathrm{Al}_{2} \mathrm{O}_{3}-$ ethanol-Si. In the absence of laser light the inequalities (48) are not satisfied (see Fig. 222). The computational results for the Casimir pressure in this case are shown in Fig. 223(b) by the black line. It is seen that the Casimir force is attractive as expected. Now let the Si plate be illuminated with the laser light. Then the dielectric permittivity of $\mathrm{Si}$ is described by the solid line labeled 2 in Fig. 22, and the inequalities (48) are satisfied. The respective computational results for the Casimir pressure versus separation are shown by the solid grey line in Fig.23(b). As can be seen in this figure, at separations $a>71.5 \mathrm{~nm}$ the corresponding Casimir force is repulsive. In this case, the Casimir repulsion and attraction are of the same order of magnitude within a wide range of separations. Thus, the third pair of plates provides an example where the illumination of the Si plate changes the Casimir attraction to the Casimir repulsion of the same order of magnitude.

The possibility to observe the pulsating Casimir force was discussed $\frac{48}{\text { for the }}$ two plates completely immersed in the liquid far away from any air-liquid interface in order to prevent the occurrence of capillary forces. It is expected that special procedures for surface preparation of the plates will be necessary to bring about intimate contact between the plates and liquid. Then the only liquid-based force is the drag force due to the movement of the plates in response to the change of the Casimir force. It can be seen, however, that the drag force is negligibly small. 
Thus, for the Casimir pressure values of around $10 \mathrm{mPa}$ and typical spring constants of $0.02 \mathrm{~N} \mathrm{~m}^{-1}$, the corresponding drag pressure from plate movement would be six orders of magnitude less in value. The proposed effect of the pulsating Casimir force can be used to actuate the periodic movement of electrodes and mirrors in electroand optomechanical devices. This can be achieved by using the standard frequency generators and modulators to select the appropriate duration and time between the laser pulses.

In fact the effect of a pulsating Casimir force is the combination of the familiar properties of three-layer systems 5 and the recently discovered $\sqrt{32 \mid 33}$ modulation of the Casimir force with light. The possibility of pulsation owing to vacuum fluctuations alone without use of mechanical springs to a large measure depends on the use of the repulsive Casimir force. According to the introductory part of this section, three-layer systems are presently the single configuration where the Casimir repulsion is realized experimentally. In this respect it is pertinent to mention that the repulsive Casimir force was recently predicted $123 \mid 124$ in the configuration of two parallel plates separated by an empty gap, one of which is made of a ferromagnetic dielectric and another of a metal described by the plasma model. By now the influence of magnetic properties of plate materials onto the Casimir force in not confirmed experimentally. Keeping in mind that magnetic films are widely used in different microelectromechanical devices, such a confirmation would have an important impact on nanotechnology.

\section{Conclusions and discussions}

In the foregoing we have considered all experiments performed up to date and a few proposals where the magnitude of the Casimir force was controlled using semiconductor test bodies. It is common knowledge that Casimir 1 discovered his famous effect for ideal metal planes and the most of modern experiments on measuring the Casimir force were performed with metallic test bodies 16 However, applications of the Casimir effect in nanotechnology, which is heavily based on the use of semiconductor materials, demand extensive study of the Casimir force in configurations with semiconductor surfaces. This opens considerable opportunities to change the magnitude of the Casimir force in a desirable way and to use this force for operation of microdevices. The first steps in this direction were made by the many performed experiments discussed in this review.

In Sec. 2 we considered the results of two pioneer experiments $26-28$ on measuring the Casimir force between an Au-coated sphere and semiconductor plates with different densities of charge carriers. Already the first experiment of this kind 2627 using a plate made of $p$-type Si demonstrated that the Casimir force was markedly different from the calculated forces both in the case of two metallic test bodies and an Au-coated sphere interacting with high-resistivity dielectric Si. In the second experiment, 28 the Casimir forces between an Au-coated sphere and two plates made of $n$-type $\mathrm{Si}$ with different charge carrier densities were directly measured in 
succession. In this way it was convincingly shown that the Casimir force depends on the density of charge carriers and this can be used to control its magnitude. In both experiments it was confirmed that the replacement of an Au plate with a $\mathrm{Si}$ plate leads to decrease in the magnitude of the Casimir force from about $25 \%$ to $35 \%$ depending on the value of separation in comparison with the case of two $\mathrm{Au}$ test bodies. Later it was shown 29 that the use of other semiconductor materials can result in even larger decrease in the magnitude of the Casimir pressure. Thus, the magnitude of the Casimir pressure between an Au plate and an ITO plate was found 29 to vary by from $40 \%$ to $50 \%$ smaller than for two Au plates. We have also discussed the experiment ${ }^{31}$ on measuring the Casimir force between a Ge plate and a Ge spherical lens with about $15 \mathrm{~cm}$ curvature radius. With respect to this experiment several disadvantages were remarked which make the obtained results for the Casimir force indefinite. Specifically, the subtracted asymptotic expression for the electric force might be not applicable at short separations, and the comparison with theory was based on the version of PFA which does not take into account surface imperfections which are always present on lenses of centimeter-size radii.

The most important experiment with semiconductor surfaces performed up to date is an experiment on the optical modulation of the Casimir force considered in Sec. 3. This was the measurement of the difference Casimir force between an Aucoated sphere and a $p$-type Si plate illuminated with laser pulses $32[33$ According to the measurement scheme employed not the individual forces, but only the difference of the forces in the presence and in the absence of light on a Si plate has been measured. Here, similar to the experiments considered in Sec. 2, the Casimir force in the absence of light on a Si plate was of about $35 \%-40 \%$ smaller in magnitude than for two Au test bodies. However, in the presence of light on a Si plate the force magnitude increased by about $4 \%-6 \%$ depending on separation. The increase and then the decrease of the force were repeated periodically in line with the laser pulses. This opens new prospective opportunities for the use of the Casimir force in the operation of micromachines.

The comparison of the measurement data of the experiment on optically modulated Casimir force with the Lifshitz theory resulted in an unexpected conclusion 3233 that if the dc conductivity of a Si plate in the absence of laser light is taken into account in the model of dielectric response the theory is excluded by the data at a $95 \%$ confidence level. If the dc conductivity of Si in the dark phase is omitted, the Lifshitz theory is found to be consistent with the data within both $95 \%$ and more narrow $70 \%$ confidence intervals. Keeping in mind that the dc conductivity is a real physical effect and that the Lifshitz theory is a fundamental theory based on first principles of quantum statistical physics and quantum electrodynamics, the obtained conclusion should be considered as challenging. The hypothesis 22 that some concepts of statistical physics concerning interaction of real and fluctuating electromagnetic fields might need a reconsideration has raised a heated debate $\frac{95}{95}$ However, the experimental data of one more experiment $\frac{83}{\text { led to }}$ the same conclusion that the Lifshitz theory is in disagreement with the data when 
the dc conductivity of a dielectric plate is taken into account .87 The attempts $37-39$ to modify the Lifshitz theory were also found to be in disagreement with the data of the experiment on the optically modulated Casimir force. Thus, this experiment takes on great significance as a test for fundamental physical theories.

In Sec. 4 we have discussed changes in the Casimir force which occur when the material of the plate undergoes a phase transition. Both already performed $\frac{44}{4}$ and proposed $^{45}$ experiments were considered. Specifically, it was observed 44 that the gradient of the Casimir force between an Au-coated sphere and a crystalline plate is greater by approximately $20 \%$ than for an amorphous plate made of the same semiconductor material (AgInSbTe). In spite of rather large deviations between the experimental data and theory in this experiment, the conclusion was made that phase transitions in semiconductor materials are very promising for the control of the Casimir force.

Another possibility to control the Casimir force discussed in Sec. 5 is connected with the use of corrugated semiconductor surfaces. We have discussed the two experiments $46 / 47$ on the measurement of the Casimir force gradient between an $\mathrm{Au}$-coated sphere and $\mathrm{Si}$ plates covered with rectangular corrugations of different profiles. These experiments demonstrated that there is a nontrivial interplay between material properties and geometrical profiles of semiconductor surfaces which cannot be taken into account with sufficient precision by using the PFA. Although the comparison of experiment and theory in Refs. 46, 47 was shown to be not complete (specifically, theory at zero temperature was used for the interpretation of the room-temperature measurement data) the obtained results show that geometrical shape of interacting semiconductor surfaces is a promising tool for the control of the Casimir force in addition to material properties.

A more sophisticated possibility for control of the Casimir force than all those listed above was considered in Sec. 6 . While in all the above experiments and proposals only the attractive Casimir force was exploited, here the attraction is replaced with repulsion and vice versa. Such a pulsating Casimir force 48 using no mechanical springs can be realized by a combination of the optical modulation experiment with the advantages of three-layer systems. As at least one of the layers, a semiconductor material which changes its charge carrier density by about five orders of magnitude under the influence of a laser pulse can be used.

The investigation of possibilities to control the Casimir force using semiconductor test bodies is presently in the very beginning and much should be done before some of them will find application in industrially produced devices. In future the influence of many different semiconductor materials with different kind of doping on the Casimir force should be explored. It will be of much interest to repeat the experiment on the optical modulation of the Casimir force with plates made of different semiconductors and in an ambient environment in order to determine the possibility of its application in microelectromechanical devices. Till the moment, the use of phase transitions in semiconductors in Casimir physics is in a very initial state. 
A great variety of phase transitions and their influence on the Casimir force will be investigated in the near future. Last but not the least is the prospect to achieve a deeper understanding of the role of charge carriers in the theory of thermal Casimir force. Such an outstanding problem, when it remains unsettled for a long time, may considerably retard further experimental developments.

\section{Acknowledgements}

This work was supported by the NSF grant PHY0970161 and DOE grant DEF010204ER46131.

1. H. B. G. Casimir, Proc. K. Ned. Akad. Wet. B51, 793 (1948).

2. P. W. Milonni, The Quantum Vacuum. An Introduction to Quantum Electrodynamics (Academic Press, San Diego, 1994).

3. V. M. Mostepanenko and N. N. Trunov, The Casimir Effect and its Applications (Clarendon Press, Oxford, 1997).

4. K. A. Milton, The Casimir Effect: Physical Manifestations of Zero-Point Energy (World Scientific, Singapore, 2001).

5. M. Bordag, G. L. Klimchitskaya, U. Mohideen and V. M. Mostepanenko, Advances in the Casimir Effect (Oxford University Press, Oxford, 2009).

6. E. M. Lifshitz, Sov. Phys. JETP 2, 73 (1956).

7. I. E. Dzyaloshinskii, E. M. Lifshitz and L. P. Pitaevskii, Sov. Phys. Usp. 4, 153 (1961).

8. N. G. van Kampen, B. R. A. Nijboer and K. Schram, Phys. Lett. A26, 307 (1968).

9. K. Schram, Phys. Lett. A43, 282 (1973).

10. D. Langbein, Solid State Comm. 12, 282 (1973).

11. V. B. Bezerra, G. L. Klimchitskaya and V. M. Mostepanenko, Phys. Rev. A66, 062112 (2002).

12. J. Schwinger, L. L. DeRaad and K. A. Milton, Ann. Phys. (N.Y.) 115, 1 (1978).

13. U. Mohideen and A. Roy, Phys. Rev. Lett. 81, 4549 (1998).

14. G. L. Klimchitskaya, A. Roy, U. Mohideen and V. M. Mostepanenko, Phys. Rev. A60, 3487 (1999).

15. B. W. Harris, F. Chen and U. Mohideen, Phys. Rev. A62, 052109 (2000).

16. G. L. Klimchitskaya, U. Mohideen and V. M. Mostepanenko, Rev. Mod. Phys. 81, 1827 (2009).

17. R. S. Decca, E. Fischbach, G. L. Klimchitskaya, D. E. Krause, D. López and V. M. Mostepanenko, Phys. Rev. D68, 116003 (2003).

18. R. S. Decca, D. López, E. Fischbach, G. L. Klimchitskaya, D. E. Krause and V. M. Mostepanenko, Ann. Phys. (N.Y.) 318, 37 (2005).

19. R. S. Decca, D. López, E. Fischbach, G. L. Klimchitskaya, D. E. Krause and V. M. Mostepanenko, Phys. Rev. D75, 077101 (2007).

20. R. S. Decca, D. López, E. Fischbach, G. L. Klimchitskaya, D. E. Krause and V. M. Mostepanenko, Eur. Phys. J. C51, 963 (2007).

21. V. B. Bezerra, G. L. Klimchitskaya, V. M. Mostepanenko and C. Romero, Phys. Rev. A69, 022119 (2004).

22. V. M. Mostepanenko and G. L. Klimchitskaya, Int. J. Mod. Phys. A25, 2302 (2010).

23. W. Arnold, S. Hunklinger and K. Dransfeld, Phys. Rev. B19, 6049 (1979).

24. N. Inui, J. Phys. Soc. Jpn. 73, 332 (2004).

25. N. Inui, J. Phys. Soc. Jpn. 75, 024004 (2006).

26. F. Chen, U. Mohideen, G. L. Klimchitskaya and V. M. Mostepanenko, Phys. Rev. A72, 020101(R) (2005); ibid A73, 019905(E) (2006). 
27. F. Chen, U. Mohideen, G. L. Klimchitskaya and V. M. Mostepanenko, Phys. Rev. A74, 022103 (2006).

28. F. Chen, G. L. Klimchitskaya, V. M. Mostepanenko and U. Mohideen, Phys. Rev. Lett. 97, 170402 (2006).

29. S. de Man, K. Heeck, R. J. Wijngaarden and D. Iannuzzi, Phys. Rev. Lett. 103, 040402 (2009).

30. S. de Man, K. Heeck and D. Iannuzzi, Phys. Rev. A82, 062512 (2010).

31. W. J. Kim, A. O. Sushkov, D. A. R. Dalvit and S. K. Lamoreaux, Phys. Rev. Lett. 103, 060401 (2009).

32. F. Chen, G. L. Klimchitskaya, V. M. Mostepanenko and U. Mohideen, Optics Express 15, 4823 (2007).

33. F. Chen, G. L. Klimchitskaya, V. M. Mostepanenko and U. Mohideen, Phys. Rev. B76, 035338 (2007).

34. B. Geyer, G. L. Klimchitskaya and V. M. Mostepanenko, Phys. Rev. D72, 085009 (2005).

35. G. L. Klimchitskaya, B. Geyer and V. M. Mostepanenko, J. Phys.: Math. Gen. A39, 6495 (2006).

36. B. Geyer, G. L. Klimchitskaya and V. M. Mostepanenko, Ann. Phys. (N.Y.) 323, 291 (2008).

37. L. P. Pitaevskii, Phys. Rev. Lett. 101, 163202 (2008).

38. D. A. R. Dalvit and S. K. Lamoreaux, Phys. Rev. Lett. 101, 163203 (2008).

39. V. B. Svetovoy, Phys. Rev. Lett. 101, 163603 (2008).

40. G. L. Klimchitskaya, U. Mohideen and V. M. Mostepanenko, J. Phys.: Math. Theor. A41, 432001(F) (2008).

41. R. S. Decca, E. Fischbach, B. Geyer, G. L. Klimchitskaya, D. E. Krause, D. López, U. Mohideen and V. M. Mostepanenko, Phys. Rev. Lett. 102, 189303 (2009).

42. B. Geyer, G. L. Klimchitskaya, U. Mohideen and V. M. Mostepanenko, Phys. Rev. Lett. 102, 189301 (2009).

43. V. M. Mostepanenko, R. S. Decca, E. Fischbach, B. Geyer, G. L. Klimchitskaya, D. E. Krause, D. López and U. Mohideen, Int. J. Mod. Phys. A24, 1721 (2009).

44. G. Torricelli, P. J. van Zwol, O. Shpak, C. Binns, G. Palasantzas, B. J. Kooi, V. B. Svetovoy and M. Wuttig, Phys. Rev. A82, 010101(R) (2010).

45. R. Castillo-Garza, C.-C. Chang, D. Jimenez, G. L. Klimchitskaya, V. M. Mostepanenko and U. Mohideen, Phys. Rev. A75, 062114 (2007).

46. H. B. Chan, Y. Bao, J. Zou, R. A. Cirelli, F. Klemens, W. M. Mansfield and C. S. Pai, Phys. Rev. Lett. 101, 030401 (2008).

47. Y. Bao, R. Guérout, J. Lussange, A. Lambrecht, R. A. Cirelli, F. Klemens, W. M. Mansfield, C. S. Pai and H. B. Chan, Phys. Rev. Lett. 105, 250402 (2010).

48. G. L. Klimchitskaya, U. Mohideen and V. M. Mostepanenko, J. Phys.: Mat. Theor. A40, F841 (2007).

49. J. Blocki, J. Randrup, W. J. Swiatecki and C. F. Tsang, Ann. Phys. (N.Y.) 105, 427 (1977).

50. B. Geyer, G. L. Klimchitskaya and V. M. Mostepanenko, Phys. Rev. A82, 032513 (2010).

51. Handbook of Optical Constants of Solids, ed. E. D. Palik (Academic, New York, 1985).

52. A. Lambrecht and S. Reynaud, Eur. Phys. J. D8, 309 (2000).

53. A. Lambrecht, I. Pirozhenko, L. Duraffourg and P. Andreucci, Europhys. Lett. 77, 44006 (2007).

54. H.-C. Chiu, C.-C. Chang, R. Castillo-Garza, F. Chen and U. Mohideen, J. Phys.: Math. Theor. A41, 164022 (2008). 
55. R. S. Decca, D. López, H. B. Chan, E. Fischbach, D. E. Krause and C. R. Jamell, Phys. Rev. Lett. 94, 240401 (2005).

56. H. B. Chan, V. A. Aksyuk, R. N. Kleiman, D.J. Bishop and F. Capasso, Science 291, 1941 (2001).

57. H. B. Chan, V. A. Aksyuk, R. N. Kleiman, D.J. Bishop and F. Capasso, Phys. Rev. Lett. 87, 211801 (2001).

58. G. Jourdan, A. Lambrecht, F. Comin and J. Chevrier, Europhys. Lett. 85, 31001 (2009).

59. H. Fujiwara and M. Kondo, Phys. Rev. B71, 075109 (2005).

60. B. Geyer, G. L. Klimchitskaya and V. M. Mostepanenko, Phys. Rev. B81, 245421 (2010).

61. S. K. Lamoreaux, Phys. Rev. Lett. 78, 5 (1997); ibid 81, 5475(E) (1998).

62. http://www.ioffe.ru/SVA/NSM

63. W. J. Kim, M. Brown-Hayes, D. A. R. Dalvit, J. H. Brownell and R. Onofrio, Phys. Rev. A78, 020101(R) (2008).

64. S. de Man, K. Heeck and D. Iannuzzi, Phys. Rev. A79, 024102 (2009).

65. R. S. Decca, E. Fischbach, G. L. Klimchitskaya, D. E. Krause, D. López, U. Mohideen and V. M. Mostepanenko, Phys. Rev. A79, 026101 (2009).

66. G. L. Klimchitskaya and V. M. Mostepanenko, "What is credible and what is incredible in the measurements of the Casimir force", arXiv:1010.2216v1.

67. V. B. Bezerra, G. L. Klimchitskaya, U. Mohideen, V. M. Mostepanenko and C. Romero, Phys. Rev. B83, 075417 (2011).

68. Q. Wei, D. A. R. Dalvit, F. C. Lombardo, F. D. Mazzitelli and R. Onofrio, Phys. Rev. A81, 052115 (2010).

69. J. H. McLeod and W. T. Sherwood, J. Opt. Soc. Am. 35, 136 (1945).

70. M. Young, Appl. Opt. 25, 1922 (1986).

71. J. M. Bernett, Meas. Sci. Technol. 3, 1119 (1992).

72. Z. Zhong, J. Mater. Process. Technol. 122, 173 (2002).

73. D. Liu, Y. Yang, L. Wang, Y. Zhuo, C. Lu, L. Yang and R. Li, Opt. Comm. 278, 240 (2007)

74. http://www.prhoffman.com/technical/scratch-dig.htm

75. http://www.ispoptics.com/PDFs/PDFCatalog/Page55.pdf

76. G. L. Klimchitskaya, "Calculation of the Casimir force between Ge test bodies in different theoretical approaches", arXiv:0902.4254v2.

77. A. O. Sushkov, W. J. Kim, D. A. R. Dalvit and S. K. Lamoreaux, Nature Phys. 7, 230 (2011).

78. R. S. Decca, E. Fischbach, G. L. Klimchitskaya, D. E. Krause, D. López and V. M. Mostepanenko, Phys. Rev. A82, 052515 (2010).

79. W. R. Smythe, Electrostatics and Electrodynamics (McGraw-Hill, New York, 1950).

80. A. J. Sabbah and D. M. Riffe, Phys. Rev. B66, 165217 (2002).

81. M. Nagai and M. Kuwata-Gonokami, J. Phys. Soc. Jap. 71, 2276 (2002).

82. S. G. Rabinovich, Measurement Errors and Uncertainties. Theory and Practice (Springer-Verlag, New York, 2000).

83. J. M. Obrecht, R. J. Wild, M. Antezza, L. P. Pitaevskii, S. Stringari and E. A. Cornell, Phys. Rev. Lett. 98, 063201 (2007).

84. T. Vogel, G. Dodel, E. Holzhauer, H. Salzmann and A. Theurer, Appl. Opt. 31, 329 (1992)

85. V. M. Mostepanenko and B. Geyer, J. Phys.: Math. Theor. A41, 164014 (2008).

86. G. L. Klimchitskaya and B. Geyer, J. Phys.: Math. Theor. A41, 164032 (2008).

87. G. L. Klimchitskaya and V. M. Mostepanenko, J. Phys.: Math. Theor. A41, 312002(F) 
(2008).

88. G. L. Klimchitskaya, M. N. Bukina, Yu. V. Churkin and V. A. Yurova, Phys. Solid State 52, 2033 (2010).

89. Yu. B. Rumer and M. S. Ryvkin, Thermodynamics, Statistical Physics and Kinetics (Mir, Moscow, 1980).

90. G. L. Klimchitskaya, J. Phys.: Conf. Ser. 161, 012002 (2009).

91. V. M. Mostepanenko, J. Phys.: Conf. Ser. 161, 012003 (2009).

92. L. P. Pitaevskii, Phys. Rev. Lett. 102, 189302 (2009).

93. D. A. R. Dalvit and S. K. Lamoreaux, Phys. Rev. Lett. 102, 189304 (2009).

94. D. A. R. Dalvit and S. K. Lamoreaux, J. Phys.: Conf. Ser. 161, 012009 (2009).

95. L. P. Pitaevskii, Int. J. Mod. Phys. A25, 2313 (2010).

96. V. B. Svetovoy, Phys. Rev. Lett. 102, 219903(E) (2009).

97. G. Bimonte, E. Calloni, G. Esposito, L. Milano and L. Rosa, Phys. Rev. Lett. 94, $180402(2005)$.

98. G. Bimonte, E. Calloni, G. Esposito and L. Rosa, Nucl. Phys. B726, 441 (2005).

99. G. Bimonte, Phys. Rev. A78, 062101 (2008).

100. P. J. van Zwol, G. Palasantzas and J. Th. M. De Hosson, Phys. Rev. B77, 075412 (2008).

101. P. J. van Zwol, V. B. Svetovoy and G. Palasantzas, Phys. Rev. B80, 235401 (2009).

102. A. Zylbersztejn and N. F. Mott, Phys. Rev. B11, 4383 (1975).

103. M. Soltani, M. Chaker, E. Haddad, R. V. Kruzelecky and D. Nikanpour, J. Vac. Sci. Technol. A22, 859 (2004).

104. J. Y. Suh, R. Lopez, L. C. Feldman and R. F. Haglund, J. Appl. Phys. 96, 1209 (2004).

105. H. W. Verleur, A. S. Barker and C. N. Berglund, Phys. Rev. 172, 788 (1968).

106. I. Pirozhenko and A. Lambrecht, Phys. Rev.. A77, 013811 (2008).

107. T. Emig, A. Hanke, R. Golestanian and M. Kardar, Phys. Rev. A67, 022114 (2003).

108. R. Büscher and T. Emig, Phys. Rev. A69, 062101 (2004).

109. A. Lambrecht and V. N. Marachevsky, Phys. Rev. Lett. 101, 160403 (2008).

110. H.-C. Chiu, G. L. Klimchitskaya, V. N. Marachevsky, V. M. Mostepanenko and U. Mohideen, Phys. Rev. B80, 121402(R) (2009).

111. H.-C. Chiu, G. L. Klimchitskaya, V. N. Marachevsky, V. M. Mostepanenko and U. Mohideen, Phys. Rev. B81, 115417 (2010).

112. B. Geyer, G. L. Klimchitskaya and V. M. Mostepanenko, Eur. Phys. J. C57, 823 (2008).

113. M. G. Silverinha, Phys. Rev. B82, 085101 (2010).

114. M. G. Silverinha and S. I. Maslovski, Phys. Rev. Lett. 105, 189301 (2010).

115. S.-W. Lee and W. M. Sigmund, J. Colloid Interface Sci. 243, 365 (2001).

116. S.-W. Lee and W. M. Sigmund, Colloids Surf. A204, 43 (2002).

117. A. Milling, P. Mulvaney and I. Larson, J. Colloid Interface Sci. 180, 460 (1996).

118. A. Meurk, P. F. Luckham and L. Bergström, Langmuir 13, 3896 (1997).

119. J. N. Munday, F. Capasso and V. A. Parsegian, Nature 457, 170 (2009).

120. B. Geyer, G. L. Klimchitskaya, U. Mohideen and V. M. Mostepanenko, Phys. Rev. A77, 036102 (2008).

121. J. N. Munday, D. Iannuzzi, Y. Barash and F. Capasso, Phys. Rev. A71, 042102 (2005).

122. L. Bergström, Adv. Coll. Interface Sci. 70, 125 (1997).

123. B. Geyer, G. L. Klimchitskaya and V. M. Mostepanenko, Phys. Rev B81, 104101 (2010).

124. G. L. Klimchitskaya, B. Geyer and V. M. Mostepanenko, Int. J. Mod. Phys. A25, 
May 29, 2022 0:51 WSPC/INSTRUCTION FILE arxSC

Control of the Casimir force using semiconductor test bodies 61

2293 (2010). 